\title{
Symmetry restoration at finite temperature with weak magnetic fields
}

\author{
Jorge Navarro ${ }^{1,2}$, Angel Sánchez ${ }^{3}$, Maria Elena Tejeda-Yeomans ${ }^{4}$, Alejandro Ayala ${ }^{1}$ and Gabriella Piccinelli ${ }^{5}$ \\ ${ }^{1}$ Instituto de Ciencias Nucleares, Universidad Nacional Autónoma de México, \\ Apartado Postal 70-543, México Distrito Federal 04510, Mexico. \\ ${ }^{2}$ Departamento de Física, Universidad del Atlántico, \\ Km. 7 antigua vía a Puerto Colombia, A.A. 1890, Barranquilla, Colombia. \\ ${ }^{3}$ Instituto de Física y Matemáticas, Universidad Michoacana de San Nicolás de Hidalgo, \\ Apartado Postal 2-82, Morelia, Michoacán 58040, México. \\ ${ }^{4}$ Departamento de Física, Universidad de Sonora, Boulevard Luis Encinas J. y Rosales, \\ Colonia Centro, Hermosillo, Sonora 83000, Mexico. \\ ${ }^{5}$ Centro Tecnológico, FES Aragón, Universidad Nacional Autónoma de México, \\ Avenida Rancho Seco S/N, Bosques de Aragón, Nezahualcóyotl, Estado de México 57130, Mexico.
}

\begin{abstract}
We study symmetry restoration at finite temperature in the standard model during the electroweak phase transition in the presence of a weak magnetic field. We compute the finite temperature effective potential up to the contribution of ring diagrams, using the broken phase degrees of freedom, and keep track of the gauge parameter dependence of the results. We show that under these conditions, the phase transition becomes stronger first order.

PACS numbers: 98.62.En, 98.80.Cq, 12.38.Cy
\end{abstract}

\section{INTRODUCTION}

The properties of the universe's primordial plasma at high temperature play an important role in the attempts to explain several outstanding questions in cosmology such as the origin of the matter-antimatter asymmetry. Starting from symmetric conditions, it was shown by Sakharov [1] that three ingredients are needed to develop a baryon asymmetry: ( $i$ ) Baryon number violation processes, (ii) $\mathrm{C}$ and $\mathrm{CP}$ violation and (iii) departure from thermal equilibrium. These conditions are met in the Minimal Standard Model (MSM) during the Electroweak Phase Transition (EWPT) provided that this be first order. It is however well known that neither the amount of $\mathrm{CP}$ violation nor the strength of the phase transition are enough to produce and subsequently preserve a possible baryon number at this stage of the universe evolution [2, 3].

A great deal of effort has been devoted to put forward viable scenarios that increase the amount of $\mathrm{CP}$ violation and/or make the EWPT stronger first order [4, 5]. At the same time, in the last years, the study of the effects of primordial magnetic fields on other cosmological processes [6 8], have also been given attention, including the very same EWPT [9, 10].

Since magnetic fields before the EWPT belong to the $U_{Y}(1)$ group, this kind of fields properly receive the name of hypermagnetic. The expectation is that in their presence, the order of the EWPT is increased, in analogy with the case of superconductivity, where an external magnetic field changes the order of the phase transition from second to first due to the Meissner effect [9, 11, 12].

The description of the physical processes able to generate magnetic fields is an old problem in cosmology [13]. The general approach is to identify mechanisms for the generation of seed fields [14, 15] which can later be amplified into fields on larger scales. For instance, certain types of inflationary models can produce magnetic fields extending over horizon distances [16]. Other possibilities make use of cosmological phase transitions [17] through formation of dipole charge layers on surfaces of phase transition bubble walls. Although at present there is no conclusive evidence about the origin of magnetic fields, their existence prior to the EWPT cannot certainly be ruled out. They have been observed in galaxies, clusters, intracluster medium and high redshift objects [18].

The presence of magnetic fields in the early universe can leave its imprint in a variety of phenomena. Magnetic fields directly interact with ionized baryons and indirectly influence photons, through the tight coupling between baryons and photons at that epoch. Cold dark matter is also indirectly affected, through gravitational interaction. Thus, a primordial magnetic field imprint can be searched for (through temperature anisotropies, statistics and polarization) in the Cosmic Microwave Background (CMB), formation of large scale structure, gravitational wave background and the nucleosynthesis processes. A homogeneous magnetic field would give rise to a dipole anisotropy in the background radiation. On this basis, Cosmic Background Explorer (COBE) results give an upper bound on the present equivalent field strength of $B_{0} \lesssim 10^{-9} \mathrm{G}[14,19]$. A lot of work has been done recently in the field of the observational constraints, considering different aspects of the interaction of primordial magnetic fields with the CMB, as well as different features and scales of these cosmic fields (see for example 20] and references therein). In particular, in a recent work [21], using both CMB and large-scale structure observational data, the authors place new constraints on primordial magnetic fields, both on their magnitude and power spectral index. This last parameter is important for the discrimination between models of magnetogenesis. For all cosmological parameters, the same priors as those adopted in the WMAP analysis are used. Standard 
cosmological parameters are mainly constrained by low multipoles, while primordial magnetic fields dominate on small angular scales (high multipoles). The degeneracy among magnetic fields parameters is broken by the different effects of primordial magnetic fields on the matter power and $\mathrm{CMB}$ radiation spectra. An interesting result of this analysis is that the probability distribution of the parameters has a maximum for nonzero values of both the magnitude and the power spectral index. Although these values are still consistent with zero magnetic fields (thus implying only upper limits on the field strength of $\sim 10^{-9}$ Gauss on a comoving scale of $1 \mathrm{Mpc}$ ) they suggest the possibility of a detection with forthcoming data.

Recall that the development of the EWPT can be described by means of the MSM effective potential (EP) where the order parameter is the Higgs vacuum expectation value $v$ [22, 23]. Starting from a very high temperature, the minimum of the theory happens for $v=0$, the so called symmetric phase. Decreasing the temperature produces the EP to develop a secondary minimum, in case the phase transition is first order. Since the phase transition does not start until the secondary minimum is degenerate with the original one, its development in the high temperature phase can be described in terms of MSM symmetry restored degrees of freedom. Particle masses depend parametrically on the order parameter and acquire their fixed values only when the phase transition is completed.

Within the particle physics calculational methods, it is common to work using the degrees of freedom in the symmetry restored phase. A drawback of this scheme is that, since the gauge boson mass matrix is not diagonal, contributions from gauge bosons to the EP are harder to compute. Another general aspect of calculations involving MSM degrees of freedom is that they are usually performed for a given value of the (covariant) gauge parameter, which makes it impossible to keep track of a gauge parameter presence, if any, in phase transition related observables.

In a recent work [24], the development of the EWPT in the MSM from the symmetric phase has been studied in the presence of a weak external hypermagnetic field up to the contribution of ring diagrams. The main result from that work is that the presence of the field strengthens the first order nature of the phase transition. The result is in agreement with calculations performed at a classical [11] and one-loop levels [12], as well as with lattice simulations [9]. On the other hand, other analytical non-perturbative approaches where the MSM finite temperature EP is studied for the case of strong magnetic fields [25, 26], reach the conclusion that these fields inhibit the first order phase transition. These works attribute the result to the contribution of light fermion masses which are generally neglected in other computations. However, they also neglect to consider the infrared cutoff provided by thermal masses, which casts doubts on their conclusions. It is thus important to study the phase transition using the broken phase degrees of freedom, since it will help to set up the stage for a detailed analysis of the phase equilibrium conditions as the EWPT develops.

In this work we generalize the analysis of the development of the EWPT in the presence of a constant magnetic field, working with the degrees of freedom in the broken symmetry phase where the symmetry of the theory has been reduced from $S U(2)_{L} \times U(1)_{Y}$ to $U(1) \mathrm{em}$. We work with an arbitrary value of the (covariant) gauge parameter in the weak field limit and up to the contribution of the ring diagrams, that have been shown to be crucial for the description of the long wavelength properties of the theory [27]. We find that there is a small gauge parameter dependence on observables such as the critical temperature and the position of the broken phase minimum, but otherwise confirm that the presence of a weak magnetic field, increases the order of the phase transition.

Also, as we will see in the next section, we work explicitly with the assumption that the hierarchy of scales $e B \ll m^{2} \ll T^{2}$ is obeyed, where we consider $m$ as a generic mass of the problem at the electroweak scale. Since at the phase transition $m \sim v$, the effective coupling in the perturbative expansion becomes $g^{2} T / v$ and thus when $v / T$ is small ( $T / v$ is large), the perturbative series is not reliable. This issue has been discussed in detail by Arnold and Espinosa [28] where they use a power counting argument whereby the leading temperature terms (after accounting for the thermal contribution to the effective mass) give rise to corrections proportional to $g^{2} T / m$. Notice however that in this work we do not attempt to claim that the hierarchy of scales that we worked with makes $v / T$ grow to the extent that both, the sphaleron erasure bound is avoided nor the perturbative expansion problem is solved. Rather, our purpose is to explore the effective potential method to see if a hint of a growth of $v / T$ is possible. As we will see, such growth does happen and in that sense the results are encouraging. Another recent study of the effective Lagrangian in the MSM using broken phase degrees of freedom at finite temperature and density can be found in Ref. [29].

The paper is organized as follows: In Sec. I we write down the MSM using the degrees of freedom in the broken symmetry phase. In Sec. III we lay down the formalism to include weak magnetic fields in the computation of charged particle propagators. In Sec. IV we work with these degrees of freedom to compute particle self-energies that are used in Sec. $\nabla$ to compute the MSM EP up to the contributions of ring diagrams. In Sec. VI we study this EP as a function of the Higgs vacuum expectation value and show that the order of the EWPT becomes stronger first order in the presence of the magnetic field. We also study the gauge parameter dependence of the phase transition parameters. Finally, we conclude and discuss our results in Sec. VII and leave for the appendices the listing of the one-loop MSM self-energies in the presence of weak magnetic fields, for arbitrary values of the gauge parameter. 


\section{MINIMAL STANDARD MODEL}

Quantization of the MSM in an arbitrary covariant gauge using the Faddeev-Popov technique involves not only physical fields such as the gauge bosons $W_{\mu}, Z_{\mu}, A_{\mu}$, the neutral Higgs $h$, and fermions, but also charged and neutral Goldstone bosons $\chi^{ \pm}, \chi_{3}$, respectively, which are fields that do not propagate asymptotically. Nevertheless, these last fields appear in loops. Working in a renormalizable gauge of the t'Hooft type, the so called $R_{\xi}$ gauge, in order to compensate contributions from the spurious components of the gauge fields, it is necessary to also introduce Faddeev-Popov ghosts for each of the gauge boson fields $\eta_{W}^{ \pm}, \eta_{Z}$ and $\eta_{\gamma}$. Thus, the Lagrangian also depends on the choice of the gauge fixing term.

In order to consider all the contributions to the MSM $\mathrm{EP}$, we write the complete Lagrangian sector by sector, in an arbitrary $R_{\xi}$ gauge, after the symmetry breaking.

$$
\mathcal{L}=\mathcal{L}_{H}+\mathcal{L}_{g b}+\mathcal{L}_{f}+\mathcal{L}_{Y}+\mathcal{L}_{g f}+\mathcal{L}_{F P},
$$

where $\mathcal{L}_{H}, \mathcal{L}_{g b}, \mathcal{L}_{f}, \mathcal{L}_{Y}, \mathcal{L}_{g f}$ and $\mathcal{L}_{F P}$ are the Lagrangians for the Higgs, gauge boson, fermion, Yukawa, gauge fixing and Faddeev-Popov sectors, respectively.

The Lagrangian for the Higgs field is

$$
\mathcal{L}_{H}=\left(D_{\mu} \Phi\right)^{\dagger}\left(D^{\mu} \Phi\right)+c^{2}\left(\Phi^{\dagger} \Phi\right)-\lambda\left(\Phi^{\dagger} \Phi\right)^{2} .
$$

The $S U(2)_{L} \times U(1)_{Y}$ symmetry of the Lagrangian in Eq. (11) is broken spontaneously by introducing an adequate Higgs scalar $\Phi$, which develops a vacuum expectation value $v$. The most economical choice for $\Phi$ is an $S U(2)_{L}$ doublet of complex fields with hypercharge (Y) +1 , given by

$$
\Phi=\frac{1}{\sqrt{2}}\left(\begin{array}{c}
\chi_{2}+i \chi_{1} \\
v+h-i \chi_{3}
\end{array}\right) .
$$

We take $h$ as the physical Higgs field with a mass given by

$$
m_{H}^{2}=3 \lambda v^{2}-c^{2} \equiv m_{4}^{2} .
$$

From the kinetic energy term on Eq. (2) one obtains the gauge boson masses by finding the mass eigenstates in the broken symmetry phase, which leads to

$$
m_{W}^{2}=g^{2} v^{2} / 4, \quad m_{Z}^{2}=\left(g^{2}+g^{\prime 2}\right) v^{2} / 4
$$

and the relations between the gauge fields $W_{\mu}^{a}, B_{\mu}$ and $W_{\mu}^{ \pm}, Z_{\mu}, A_{\mu}$ before and after symmetry breaking, respectively

$$
\begin{aligned}
W_{\mu}^{ \pm} & =\frac{1}{\sqrt{2}}\left(W_{\mu}^{1} \mp i W_{\mu}^{2}\right), \\
Z_{\mu} & =\frac{1}{\sqrt{g^{2}+g^{\prime 2}}}\left(g W_{\mu}^{3}-g^{\prime} B_{\mu}\right), \\
A_{\mu} & =\frac{1}{\sqrt{g^{2}+g^{\prime 2}}}\left(g^{\prime} W_{\mu}^{3}+g B_{\mu}\right) .
\end{aligned}
$$

Hereafter $g$ and $g^{\prime}$ are the $S U(2)_{L}$ and $U(1)_{Y}$ gauge couplings, respectively.

In order to obtain the interaction terms between the Higgs field and the gauge bosons, it is convenient to rewrite the covariant derivative

$$
D_{\mu}=\partial_{\mu}+i g \frac{\tau^{a}}{2} W_{\mu}^{a}+i \frac{g^{\prime} \mathbf{Y}}{2} B_{\mu}^{\prime},
$$

in terms of the mass eigenstates where $B_{\mu}^{\prime}=B_{\mu}+B_{\mu}^{\text {ext }}$. This is explicitly accomplished by applying $D_{\mu}$ in terms of the states defined in Eq. (6), to the Higgs field in Eq. (3). On the other hand, the self-interactions of the Higgs field, are obtained from the scalar potential in Eq. (2).

The kinetic energy from the $S U(2)_{L}$ and $U(1)_{Y}$ gauge bosons is

$$
\mathcal{L}_{g b}=-\frac{1}{4} \mathbf{F}^{\mu \nu} \cdot \mathbf{F}_{\mu \nu}-\frac{1}{4} B^{\prime \mu \nu} B_{\mu \nu}^{\prime}
$$

where

$$
\begin{aligned}
& \mathbf{F}_{\mu \nu}=\partial_{\mu} \mathbf{W}_{\nu}-\partial_{\nu} \mathbf{W}_{\mu}-g \mathbf{W}_{\mu} \times \mathbf{W}_{\nu} \\
& B_{\mu \nu}^{\prime}=\partial_{\mu} B_{\nu}^{\prime}-\partial_{\nu} B_{\mu}^{\prime} .
\end{aligned}
$$

After symmetry breaking and introduction of the mass eigenstates, one obtains from Eq. (8) the kinetic term as well as interaction terms involving three and four gauge fields.

The Lagrangian for the fermion sector is

$$
\mathcal{L}_{f}=\bar{\Psi}_{R} \not D \Psi_{R}+\bar{\Psi}_{L} \not D \Psi_{L},
$$

where $\Psi_{L, R}=\frac{1}{2}\left(1 \mp \gamma_{5}\right) \Psi$. Once again, the fermion-gauge boson interactions are obtained by writing the covariant derivative in terms of the mass eigenstates after symmetry breaking.

We work in the limit where all fermion masses are negligible, except the top quark mass. Therefore, the main contribution to the Yukawa sector is

$$
\mathcal{L}_{Y}=y_{t} \overline{q_{L}} \tilde{\Phi} t_{R}+\text { h.c. }
$$

where $y_{t}$ is the top quark Yukawa coupling, $q_{L}$ is the third family quark doublet, $t_{R}$ is the right handed top quark, $\tilde{\Phi}=i \sigma_{2} \Phi^{*}$ and $\sigma_{2}$ is the second Pauli matrix. After symmetry breaking this Lagrangian yields for the top quark mass $m_{t}=\frac{y_{t}}{\sqrt{2}} v$. To determine the interaction terms between fermions and the Higgs field, it is necessary to rotate the fermions to the mass eigenbasis.

In the $R_{\xi}$ gauge, the gauge fixing Lagrangian is

$$
\begin{aligned}
\mathcal{L}_{g f}= & -\frac{1}{2 \xi}\left(\partial^{\mu} W_{\mu}^{i}-\frac{1}{2} \xi g v \chi^{i}\right)^{2} \\
& -\frac{1}{2 \xi}\left(\partial^{\mu} Z_{\mu}-\frac{1}{2} \xi g^{\prime} v \chi_{3}\right)^{2} \\
& -\frac{1}{2 \xi}\left(\partial^{\mu} A_{\mu}\right)^{2}
\end{aligned}
$$


where $i=1,2$ and $\xi$ is the gauge parameter (which for simplicity we set it to be the same for all gauge bosons) and $m_{i}$ stands for the Goldstone boson masses, given by

$$
m_{1}^{2}=m_{2}^{2}=\lambda v^{2}-c^{2}+\xi g^{2} \frac{v^{2}}{4}
$$

and

$$
m_{3}^{2}=\lambda v^{2}-c^{2}+\xi\left(g^{2}+g^{\prime 2}\right) \frac{v^{2}}{4},
$$

respectively.

Since we work with an arbitrary value of the gauge fixing parameter $\xi$, the ghost fields acquire masses proportional to the corresponding gauge boson ones $m_{g b}$

$$
m_{\eta_{g b}}^{2}=\xi m_{g b}^{2} \text {. }
$$

Notice that these fields do not propagate asymptotically, thus appear only in internal Feynman diagram lines.

In order to find the corresponding Faddeev-Popov ghost interactions, the ghost fields are rotated like the gauge fields in Eq. (6). We emphasize that by working with an arbitrary value of $\xi$, the ghost fields do contribute to the $v$-dependent part of the one-loop EP. Note that the EP is in principle a gauge dependent object [30], however, physical quantities obtained from it should be gauge independent [31].

\section{CHARGED PARTICLE PROPAGATORS IN THE PRESENCE OF A MAGNETIC FIELD}

We work with MSM degrees of freedom in the symmetry broken phase, where the external magnetic field belongs to the $U(1)$ em group. To include the effect of the external field, we use Schwinger's proper time method [32]. In the broken phase, we have three kinds of charged particles that couple to the external field, namely: scalars, fermions and gauge bosons, whose propagators are

$$
\begin{aligned}
D_{B}\left(x, x^{\prime}\right) & =\phi\left(x, x^{\prime}\right) \int \frac{d^{4} k}{(2 \pi)^{4}} e^{-i k \cdot\left(x-x^{\prime}\right)} D_{B}(k), \\
S_{B}\left(x, x^{\prime}\right) & =\phi\left(x, x^{\prime}\right) \int \frac{d^{4} k}{(2 \pi)^{4}} e^{-i k \cdot\left(x-x^{\prime}\right)} S_{B}(k), \\
G_{B}^{\mu \nu}\left(x, x^{\prime}\right) & =\phi\left(x, x^{\prime}\right) \int \frac{d^{4} k}{(2 \pi)^{4}} e^{-i k \cdot\left(x-x^{\prime}\right)} G_{B}^{\mu \nu}(k),
\end{aligned}
$$

respectively. The phase factor $\phi\left(x, x^{\prime}\right)$, that breaks translation invariance, is given by

$$
\phi\left(x, x^{\prime}\right) \equiv e^{i e \int_{x^{\prime}}^{x} d \xi^{\mu}\left[B_{\mu}^{\mathrm{ext}}+\frac{1}{2} F_{\mu \nu}\left(\xi-x^{\prime}\right)^{\nu}\right]},
$$

where the vector potential $B_{\mu}^{\text {ext }}=\frac{B}{2}(0, y,-x, 0)$ gives rise to a constant magnetic field of strength $B$ along the $\hat{z}$ axis and $F_{\mu \nu}^{\text {ext }}=\partial_{\mu} B_{\nu}^{\text {ext }}-\partial_{\nu} B_{\mu}^{\text {ext }}$ is the external field strength tensor.
The momentum dependent functions $D_{B}(k), S_{B}(k)$ and $G_{B}^{\mu \nu}(k)$ are given by

$$
\begin{aligned}
i D_{B}(k) & =\int_{0}^{\infty} \frac{d s}{\cos e B s} \\
& \times \exp \left\{i s\left(k_{\|}^{2}-k_{\perp}^{2} \frac{\tan e B s}{e B s}-m^{2}+i \epsilon\right)\right\},
\end{aligned}
$$

$$
\begin{aligned}
i S_{B}(k) & =\int_{0}^{\infty} \frac{d s}{\cos e B s} \\
& \times \exp \left\{i s\left(k_{\|}^{2}-k_{\perp}^{2} \frac{\tan e B s}{e B s}-m_{f}^{2}+i \epsilon\right)\right\} \\
& \times\left[\left(m_{f}+\not k_{\|}\right) e^{i e B s \sigma_{3}}-\frac{\not k_{\perp}}{\cos e B s}\right],
\end{aligned}
$$

and

$$
\begin{aligned}
i G_{B}^{\mu \nu}(k) & =\int_{0}^{\infty} \frac{d s}{\cos e B s} e^{i s\left(k_{\|}^{2}-k_{\perp}^{2} \frac{\tan e B s}{e B s}\right)} \\
& \times\left\{e^{-i s\left(m_{g b}^{2}-i \epsilon\right)}\left[-g_{\|}^{\mu \nu}+\left(e^{2 e F s}\right)_{\perp}^{\mu \nu}\right]\right. \\
& +\left(\frac{e^{-i s\left(m_{g b}^{2}-i \epsilon\right)}-e^{-i s\left(\xi m_{g b}^{2}-i \epsilon\right)}}{m_{g b}^{2}}\right) \\
& \times\left[\left(k^{\mu}+k_{\lambda} F^{\mu \lambda}((\tan (e B s)) / B)\right)\right. \\
& \times\left(k^{\nu}+k_{\rho} F^{\rho \nu}((\tan (e B s)) / B)\right) \\
& \left.\left.-i \frac{e}{2}\left(F^{\mu \nu}+g_{\perp}^{\mu \nu} B \tan (e B s)\right)\right]\right\} .
\end{aligned}
$$

We use the metric tensor $g^{\mu \nu}=\operatorname{diag}(1,-1,-1,-1)$ in which $g^{\mu \nu}=g_{\|}^{\mu \nu}-g_{\perp}^{\mu \nu}$ and the notation $k_{\|}^{2}=k_{0}^{2}-k_{3}^{2}$, $k_{\perp}^{2}=k_{1}^{2}+k_{2}^{2}$, and $\sigma^{3}=i \gamma^{1} \gamma^{2}=-\gamma^{5} \not h \not$. We also use

$$
\left(e^{2 e F s}\right)_{\perp}^{\mu \nu}=g_{\perp}^{\mu \nu} \cos (2 e B s)-F^{\mu \nu} \frac{\sin (e B s)}{B}
$$

where throughout $u^{\mu}$ and $b^{\mu}$ are four-vectors describing the plasma rest frame and the direction of the magnetic field, respectively. In the rest frame, these are given by

$$
u=(1,0,0,0), \quad b=(0,0,0,1) .
$$

It has been shown that, by deforming the contour of integration, Eqs. (20) and (21) can be written as [33, 34]

$$
\begin{aligned}
& i D_{B}(k)=2 i \sum_{l=0}^{\infty} \frac{(-1)^{l} L_{l}\left(\frac{2 k_{\perp}^{2}}{e B}\right) \mathrm{e}^{-\frac{k_{\perp}^{2}}{e B}}}{k_{\|}^{2}-(2 l+1) e B-m^{2}+i \epsilon}, \\
& i S_{B}(k)=i \sum_{l=0}^{\infty} \frac{d_{l}\left(\frac{k_{\perp}^{2}}{e B}\right) D+d_{l}^{\prime}\left(\frac{k_{\perp}^{2}}{e B}\right) \bar{D}}{k_{\|}^{2}-2 l e B-m_{f}^{2}+i \epsilon}+\frac{\not k_{\perp}}{k_{\perp}^{2}},
\end{aligned}
$$

where $d_{l}(\alpha) \equiv(-1)^{n} e^{-\alpha} L_{l}^{-1}(2 \alpha), d_{n}^{\prime}=\partial d_{n} / \partial \alpha$,

$$
\begin{aligned}
& D=\left(m_{f}+\not k_{\|}\right)+\not k_{\perp} \frac{m_{f}^{2}-k_{\|}^{2}}{k_{\perp}^{2}}, \\
& \bar{D}=\gamma_{5} \not \mu \not\left(m_{f}+\not k_{\|}\right),
\end{aligned}
$$


and $L_{l}, L_{l}^{m}$ are Laguerre and Associated Laguerre polynomials, respectively. It can also be shown that by performing a similar analysis as in Refs. 33, 34, Eq. (22) can be written as 35.

$$
\begin{aligned}
& i G_{B}^{\mu \nu}(k)=\sum_{\lambda=-1}^{1} \sum_{l=0}^{\infty} \frac{2 i(-1)^{l} \mathrm{e}^{-\frac{k_{\perp}^{2}}{e B}}}{k_{\|}^{2}-(2 l+2 \lambda+1) e B-m_{g b}^{2}+i \epsilon} \\
& \times\left[T^{\mu \nu}+\frac{m_{g b}^{2}(1-\xi) P^{\mu \nu}}{k_{\|}^{2}-(2 l+2 \lambda+1) e B-\xi m_{g b}^{2}+i \epsilon}\right] \\
& \quad \times L_{l}\left(\frac{2 k_{\perp}^{2}}{e B}\right)
\end{aligned}
$$

where

$$
\begin{aligned}
T^{\mu \nu} & =\sum_{\lambda=-1}^{1}\left[g^{\mu \nu}(|\lambda|-1)\right. \\
& \left.-2 g_{\perp}^{\mu \nu} \frac{(|3 \lambda|-2)}{(2 i)^{2}}+2 \frac{F^{\mu \nu}}{B}\left(\frac{\lambda}{4 i}\right)\right], \\
P^{\mu \nu} & =\frac{1}{m_{g b}^{2}}\left[\left(k^{\mu} k^{\nu}-i \frac{e}{2} F^{\mu \nu}\right)+\left(\frac{k_{\lambda} k^{\nu} F^{\mu \lambda}}{B}\right.\right. \\
& \left.+\frac{k_{\rho} k^{\mu} F^{\rho \nu}}{B}+i \frac{e B}{2} g_{\perp}^{\mu \nu}\right)(i e B) \frac{d}{d k_{\perp}^{2}} \\
& \left.+\left(\frac{k_{\lambda} k_{\rho} F^{\mu \lambda} F^{\rho \nu}}{B^{2}}\right)(i e B)^{2} \frac{d^{2}}{d\left(k_{\perp}^{2}\right)^{2}}\right] .
\end{aligned}
$$

In order to determine the appropriate order of energy scales during the development of the the EWPT, one resorts to bounds on the strength of the magnetic fields imposed by cosmological processes in the early universe.
The relation between the strength of large scale magnetic fields and temperature is obtained from the requirement that the magnetic energy density $\rho_{\text {mag }} \sim B^{2}$ should be smaller than the overall radiation energy density $\rho_{\text {rad }} \sim T^{4}$ at nucleosynthesis, in order to preserve the estimated abundances of light elements. With this, one obtains the simple bound $B \lesssim T^{2}[36$. Furthermore, to guarantee stability conditions against the formation of a $W$-condensate [37], one obtains that the field is also weak compared to $m_{W}^{2}$. We want to study the EWPT transition via the analysis of the EP when its minima change in the presence of magnetic fields, we will work explicitly with the assumption that the hierarchy of scales

$$
e B \ll m^{2} \ll T^{2},
$$

is obeyed, where we consider $m$ as a generic mass of the problem at the electroweak scale. It is important to address the same problem with the hierarchy of the mass and the magnetic field scales switched and this will be done elsewhere 38]. We can thus perform a weak field expansion in Eqs. (25), (26) and (28) which allows us to carry out the summation over Landau levels to write the scalar, fermion and gauge boson propagators as power series in $e B$, that up to order $(e B)^{2}$ read as 33 35]

$$
\begin{gathered}
D_{B}(k)=\frac{1}{k^{2}-m^{2}}\left(1-\frac{(e B)^{2}}{\left(k^{2}-m^{2}\right)^{2}}-\frac{2(e B)^{2} k_{\perp}^{2}}{\left(k^{2}-m^{2}\right)^{3}}\right), \\
S_{B}(k)=\frac{\not k+m_{f}}{\not k^{2}-m_{f}^{2}}+\frac{\gamma_{5} \not h \not b\left(k_{\|}+m_{f}\right)(e B)}{\left(k^{2}-m_{f}^{2}\right)^{3}} \\
-\frac{2(e B)^{2} k_{\perp}^{2}}{\left(k^{2}-m_{f}^{2}\right)^{4}}\left(m_{f}+\not k_{\|}+\not k_{\perp} \frac{m_{f}^{2}-k_{\|}^{2}}{k_{\perp}^{2}}\right)
\end{gathered}
$$

and

$$
\begin{aligned}
G_{B}^{\mu \nu}(k) & =-i\left(\frac{g^{\mu \nu}-(1-\xi) \frac{k^{\mu} k^{\nu}}{k^{2}-\xi m_{g b}^{2}}}{k^{2}-m_{g b}^{2}}\right)-(e B)\left[\frac{k_{\rho}}{m_{g b}^{2}}\left(k^{\nu} \frac{F^{\mu \rho}}{B}+k^{\mu} \frac{F^{\rho \nu}}{B}\right)\right. \\
& \left.\times\left(\frac{1}{\left(k^{2}-m_{g b}^{2}\right)^{2}}-\frac{1}{\left(k^{2}-\xi m_{g b}^{2}\right)^{2}}\right)-\frac{F^{\mu \nu}}{B}\left(\frac{2}{\left(k^{2}-m_{g b}^{2}\right)^{2}}+\frac{(1-\xi)}{2\left(k^{2}-m_{g b}^{2}\right)\left(k^{2}-\xi m_{g b}^{2}\right)}\right]\right) \\
& +i(e B)^{2}\left[\frac{g^{\mu \nu}+4 g_{\perp}^{\mu \nu}}{\left(k^{2}-m_{g b}^{2}\right)^{3}}+\frac{2 g^{\mu \nu} k_{\perp}^{2}}{\left(k^{2}-m_{g b}^{2}\right)^{4}}-\frac{k^{\mu} k^{\nu}}{m_{g b}^{2}}\left(\frac{1}{\left(k^{2}-m_{g b}^{2}\right)^{3}}-\frac{1}{\left(k^{2}-\xi m_{g b}^{2}\right)^{3}}\right)\right. \\
& -2 \frac{k^{\mu} k^{\nu}}{m_{g b}^{2}} k_{\perp}^{2}\left(\frac{1}{\left(k^{2}-m_{g b}^{2}\right)^{4}}-\frac{1}{\left(k^{2}-\xi m_{g b}^{2}\right)^{4}}+\frac{g_{\perp}^{\mu \nu}}{2 m_{g b}^{2}}\left(\frac{1}{\left(k^{2}-m_{g b}^{2}\right)^{2}}-\frac{1}{\left(k^{2}-\xi m_{g b}^{2}\right)^{2}}\right)\right. \\
& \left.-\frac{2}{m_{g b}^{2}}\left(\frac{k_{\lambda} F^{\mu \lambda} k_{\rho} F^{\rho \nu}}{B^{2}}\right)^{2}\left(\frac{1}{\left(k^{2}-m_{g b}^{2}\right)^{3}}-\frac{1}{\left(k^{2}-m_{g b}^{2}\right)^{3}}\right]\right]
\end{aligned}
$$


respectively. Notice that for the assumed hierarchy of energy scales to be valid, one should stay away from working with the value for the gauge parameter $\xi=0$, since, according to Eq. (15) this choice would lead to the vanishing of the ghosts masses.

\section{SELF-ENERGIES}

In this section we compute the MSM self-energies that are in turn used for the computation of the ring diagrams in the EP.

It is well known that in the absence of an external magnetic field, the MSM thermal self-energies are gauge independent when considering only the leading contributions in temperature [39]. However, as we will show, when considering the effects of a weak external magnetic field, these self-energies turn out to be gauge dependent.

In what follows, we work in the imaginary-time formalism of thermal field theory. First, we note that the integration over four-momenta is carried out in Euclidean space with $k_{0}=i k_{4}$, this means that

$$
\int \frac{d^{4} k}{(2 \pi)^{4}} \rightarrow i \int \frac{d^{4} k_{E}}{(2 \pi)^{4}} .
$$

Next, we recall that boson energies take on discrete values, namely $k_{4}=\omega_{n}=2 n \pi T$ with $n$ an integer, and thus

$$
\int \frac{d^{4} k_{E}}{(2 \pi)^{4}} \rightarrow T \sum_{n} \int \frac{d^{3} k}{(2 \pi)^{3}} .
$$

\section{A. Higgs boson}

Figure 1 shows the diagrams that contribute to the Higgs boson self-energies affected by the magnetic field. Let us explicitly compute the momentum independent diagram shown in Fig. 1 $(a)$ for a single scalar field running in the loop, $\Pi_{a}^{H-S}$. In the weak field limit, its expression is

$$
\Pi_{a}^{H-S}=\frac{\lambda}{4} T \sum_{n} \int \frac{d^{3} \mathbf{k}}{(2 \pi)^{3}} D_{B}\left(\omega_{n}, \mathbf{k} ; m^{2}\right),
$$

where $D_{B}$ is given by Eq. (31).

Using the Euclidean version of Eq. (31), we have

$$
\begin{aligned}
\Pi_{a}^{H-S} & =2 \lambda T \sum_{n} \int \frac{d^{3} \mathbf{k}}{(2 \pi)^{3}} \frac{1}{\left(\omega_{n}^{2}+\mathbf{k}^{2}+m^{2}\right)} \\
& \times\left(1-\frac{(e B)^{2}}{\left(\omega_{n}^{2}+\mathbf{k}^{2}+m^{2}\right)^{2}}\right. \\
& \left.+\frac{2 \mathbf{k}_{\perp}^{2}(e B)^{2}}{\left(\omega_{n}^{2}+\mathbf{k}^{2}+m^{2}\right)^{3}}\right) .
\end{aligned}
$$

The integrand in Eq. (37) contains terms whose general form is

$$
I_{\alpha}\left(\mathbf{k} ; m^{2}\right) \equiv \frac{1}{\left[\omega_{n}^{2}+\mathbf{k}^{2}+m^{2}\right]^{\alpha}} .
$$

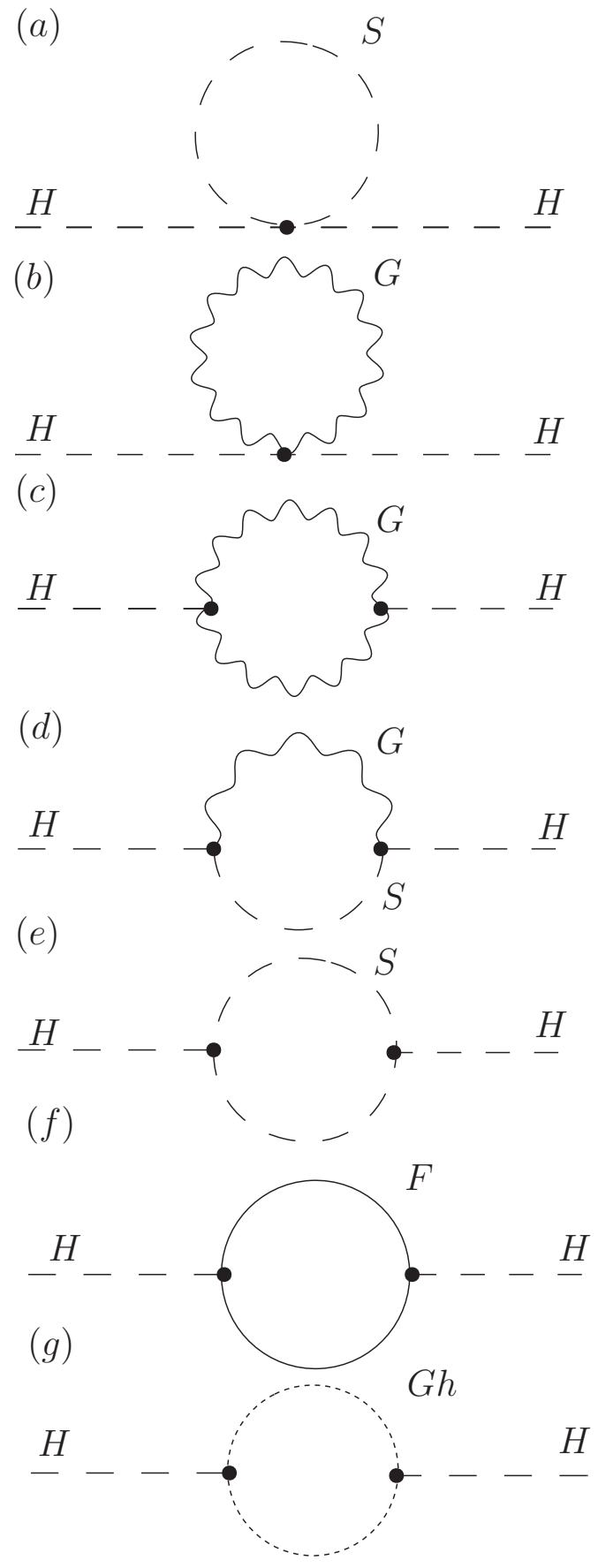

$$
\begin{aligned}
& S: \text { Higgs and Goldstone bosons } \\
& G: \mathrm{Z} \text { and W gauge bosons } \\
& F: \text { fermions } \\
& G h: \text { ghosts }
\end{aligned}
$$

FIG. 1. Feynman diagrams for the Higgs boson self-energy.

The integral involving the sum over Matsubara frequencies is performed by resorting to Refs. [40, 41]. For the 
terms with $n \neq 0$ the result is

$$
\begin{aligned}
T \sum_{n} \int \frac{d^{d} k}{(2 \pi)^{d}} \mathbf{k}^{2 a} \omega_{n}^{2 t} I_{\alpha}\left(\mathbf{k} ; m^{2}\right)=\frac{(2 T)}{(4 \pi)^{d / 2} \Gamma(\alpha)} \\
\times(2 \pi T)^{d+2 a+2 t-2 \alpha} \frac{\Gamma\left(\frac{d}{2}+a\right)}{\Gamma\left(\frac{d}{2}\right)} \mu^{2 \epsilon} \\
\quad \times \sum_{j=0}^{\infty} \frac{(-1)^{j}}{j !} \zeta\left(2\left(j+\alpha-t-\frac{d}{2}-a\right)\right) \\
\times \Gamma\left(j+\alpha-\frac{d}{2}-a\right)\left(\frac{m}{2 \pi T}\right)^{2 j}
\end{aligned}
$$

whereas for the term $n=0$ we get

$$
\begin{aligned}
T \int \frac{d^{d} k}{(2 \pi)^{d}} & \frac{\mathbf{k}^{2 a}}{\left(\mathbf{k}^{2}+m^{2}\right)^{\alpha}}=\frac{T}{(4 \pi)^{d / 2}} \frac{\Gamma\left(\frac{d}{2}+a\right)}{\Gamma\left(\frac{d}{2}\right)} \\
& \times \frac{\Gamma\left(\alpha-\frac{d}{2}-a\right)}{\Gamma(\alpha)}\left(\frac{1}{m^{2}}\right)^{\alpha-\frac{d}{2}-a},
\end{aligned}
$$

where $\zeta$ is the modified Riemann Zeta function, $\mu$ is the energy scale of dimensional regularization and $d=3-2 \epsilon$.

In terms of Eq. (38), Eq. (37) is written as

$$
\begin{aligned}
\Pi_{a}^{H-S} & =2 \lambda T \sum_{n} \int \frac{d^{3} \mathbf{k}}{(2 \pi)^{3}}\left[I_{1}\left(\mathbf{k} ; m_{i}^{2}\right)\right. \\
& \left.-\frac{(e B)^{2}}{3}\left(I_{3}\left(\mathbf{k} ; m_{i}^{2}\right)-2 \mathbf{k}^{2} I_{4}\left(\mathbf{k} ; m_{i}^{2}\right)\right)\right] .
\end{aligned}
$$

Using Eq. (39) for the terms with $n \neq 0$ and Eq. (40) for $n=0$, and after considering the contributions from all scalar fields running in the loop, we get

$$
\begin{aligned}
\Pi_{a}^{H}= & \lambda \frac{T^{2}}{2}\left[1-\frac{1}{2 \pi T}\left(2 m_{1}+m_{3}+3 m_{4}\right)\right] \\
& -\lambda \frac{(e B)^{2}}{48 \pi m_{1}^{2}}\left[\frac{T}{m_{1}}+\frac{\zeta(3) m_{1}^{2}}{4 \pi^{3} T^{2}}\right]
\end{aligned}
$$

where $m_{i}$ stands for the Goldstone boson masses, given by Eq. (13).

The contribution from the diagram in Fig. 1 $1(b)$ for a $W$ boson running in the loop is

$$
\Pi_{b}^{H-W}=\frac{g^{2}}{2} T \sum_{n} \int \frac{d^{3} \mathbf{k}}{(2 \pi)^{3}} G_{B \mu}^{\mu}\left(\omega_{n}, \mathbf{k} ; m_{W}\right) .
$$

We proceed to compute Eq. (43), as well as the rest of the self-energies, in the infrared limit [42] $q_{0}=0, \mathbf{q} \rightarrow 0$, unless explicitly stated.

In terms of the function $I_{\alpha}$ defined in Eq. (38) we can write Eq. (43) as

$$
\begin{aligned}
\Pi_{b}^{H-W} & =\frac{g^{2}}{2 m_{W}^{2}} T \sum_{n} \int \frac{d^{3} \mathbf{k}}{(2 \pi)^{3}}\left\{4 m_{W}^{2} I_{1}\left(\mathbf{k} ; m_{W}^{2}\right)\right. \\
& +\left(\omega_{n}^{2}+\mathbf{k}^{2}\right)\left[I_{1}\left(\mathbf{k} ; m_{W}^{2}\right)-I_{1}\left(\mathbf{k} ; \xi m_{W}^{2}\right)\right] \\
& +\frac{(e B)^{2}}{3}\left[-6\left(I_{2}\left(\mathbf{k} ; m_{W}^{2}\right)-I_{2}\left(\mathbf{k} ; \xi m_{W}^{2}\right)\right)\right. \\
& -\left(3 \omega_{n}^{2}+7 \mathbf{k}^{2}\right)\left(I_{3}\left(\mathbf{k} ; m_{W}^{2}\right)-I_{3}\left(\mathbf{k}, \xi m_{W}^{2}\right)\right) \\
& +4 \mathbf{k}^{2}\left(\omega_{n}^{2}+\mathbf{k}^{2}\right)\left(I_{4}\left(\mathbf{k} ; m_{W}^{2}\right)-I_{4}\left(\mathbf{k} ; \xi m_{W}^{2}\right)\right) \\
& \left.\left.+36 m_{W}^{2} I_{3}\left(\mathbf{k} ; m_{W}^{2}\right)+16 \mathbf{k}^{2} m_{W}^{2} I_{4}\left(\mathbf{k} ; m_{W}^{2}\right)\right]\right\} .
\end{aligned}
$$

Using Eq. (39) for the terms with $n \neq 0$ and Eq. (40) for the terms with $n=0$ and including all contributions from the gauge bosons in the loop, we get

$$
\begin{aligned}
\Pi_{b}^{H}= & \frac{T^{2}}{16}\left[g^{2}(3+\xi)+g^{\prime 2}\left(1+\frac{\xi}{3}\right)\right. \\
& \left.-\frac{3+\xi^{3 / 2}}{\pi T}\left(2 g^{2} m_{W}+\left(g^{2}+g^{\prime 2}\right) m_{Z}\right)\right] \\
& +\frac{(e B)^{2} g^{2}}{64 \pi m_{W}^{2}}\left[\left(3+\frac{35}{3 \xi^{1 / 2}}\right) \frac{T}{m_{W}}+\frac{11 \zeta(3) m_{W}^{2}}{3 \pi^{3} T^{2}}\right] .
\end{aligned}
$$

In a similar fashion the contributions from the diagrams $(c)$ to $(g)$ in Fig. 1 in the infrared limit, can be computed and are given Appendix A for an arbitrary value of the gauge parameter $\xi$.

The leading contribution to the Higgs self-energy is obtained by adding expressions (a)-(g) in Appendix A, keeping the leading term for each contribution. The result is gauge parameter independent and is explicitly given by

$$
\Pi_{1}=\frac{T^{2}}{4}\left\{\frac{3}{4} g^{2}+\frac{1}{4} g^{2}+2 \lambda+f^{2}\right\} .
$$

Notice that for large values of the top quark mass, namely a large coupling constant $f$, a perturbative calculation is not entirely justified. Nevertheless, here we consider our calculation as an analytical tool to explore this nonperturbative domain.

In expressions (a)-(g) for the Higgs self-energy in Appendix A, we have kept terms representing the leading contribution of each kind arising in the calculation, namely, terms that after extracting a $T^{2}$ factor, are of or$\operatorname{der}(e B)^{2} / T^{4}, m / T, v^{2} / T m$ and $(e B)^{2} / T m^{3}$, where $m$ is a generic mass. For the hierarchy of scales considered, the first kind of terms can be safely neglected. Also, terms of order $m / T$ are small. These kind of terms include ratios of gauge boson masses to the temperature, which according to Eq. (5) leads to ratios of $v / T$. When the masses appear in the denominator and are the scalar ones, the terms are potentially dangerous since their square can become negative. However, as we will soon show, these 
kind of terms are naturally canceled in the effective potential and substituted by terms where the mass that contributes is the thermal one, defined by

$$
\tilde{m}_{i}=\sqrt{m_{i}^{2}+\Pi_{1}} \text {. }
$$

When the gauge boson masses appear in the denominators throughout, one should recall that such masses are to be thought of as being computed in the broken phase. However, since our analysis considers the effective potential as a function of $v \geq 0$, we will implement such restriction by replacing in the effective potential the gauge boson masses appearing in denominators by the thermal ones, whose definition, in analogy to the scalar case, is given as the square root of the sum of the gauge boson mass squared and the leading term for the corresponding self-energy, to be defined shortly [see Eqs. (62) and (63)].

\section{B. Gauge bosons}

To express the gauge boson self-energies, in the presence of the external field, we notice that we have three independent vectors to our disposal to form tensor structures transverse to the gauge boson momentum $q^{\mu}$, namely $q^{\mu}, u^{\mu}$ and $b^{\mu}$, where these last two vectors are given in the rest frame by Eq. (24). This means that in general, these self-energies can be written as linear combinations of nine independent structures [43, 44]. Since we are interested in considering the infrared limit, $q_{0}=0, \mathbf{q} \rightarrow 0$, only $u^{\mu}$ and $b^{\mu}$ remain. Notice that the correct symmetry property for the self-energy is $\Pi^{\mu \nu}(q)=\Pi^{\nu \mu}(-q)$ 45. However, in the infrared limit, this condition means that the self-energy must be symmetric under the exchange of the Lorentz indices and therefore we can write:

$$
\Pi^{\mu \nu}=\Pi^{Q} Q^{\mu \nu}+\Pi^{R} R^{\mu \nu}+\Pi^{S} S^{\mu \nu}+\Pi^{M} g^{\mu \nu},
$$

where

$$
\begin{aligned}
Q^{\mu \nu} & =u^{\mu} u^{\nu}, \\
R^{\mu \nu} & =b^{\mu} b^{\nu}, \\
S^{\mu \nu} & =u^{\mu} b^{\nu}+u^{\nu} b^{\mu},
\end{aligned}
$$

and the transversality condition $q_{\mu} \Pi^{\mu \nu}=0$ is trivially satisfied in the infrared limit. Also, note that working in the rest frame of the medium [see Eq. (24)],

$$
\Pi^{00}=\Pi^{Q}+\Pi^{M} .
$$

Figure 2 shows the gauge boson self-energy diagrams, which involve Goldstone bosons, fermions as well as gauge bosons in the loop. Let us explicitly compute the component $\Pi_{a}^{00}$ for the self-energy diagram shown in Fig. 2(a) for the case where the external particle and the loop particle are $W$-bosons. The explicit expression for all the components of the tensor is

$$
\begin{aligned}
\Pi_{(a)}^{\mu \nu W}(q) & =i g^{2}\left(2 g^{\mu \rho} g^{\nu \sigma}-g^{\mu \nu} g^{\rho \sigma}-g^{\mu \sigma} g^{\rho \nu}\right) \\
& \times \int \frac{d^{4} k}{(2 \pi)^{4}} G_{B \rho \sigma}(k) .
\end{aligned}
$$
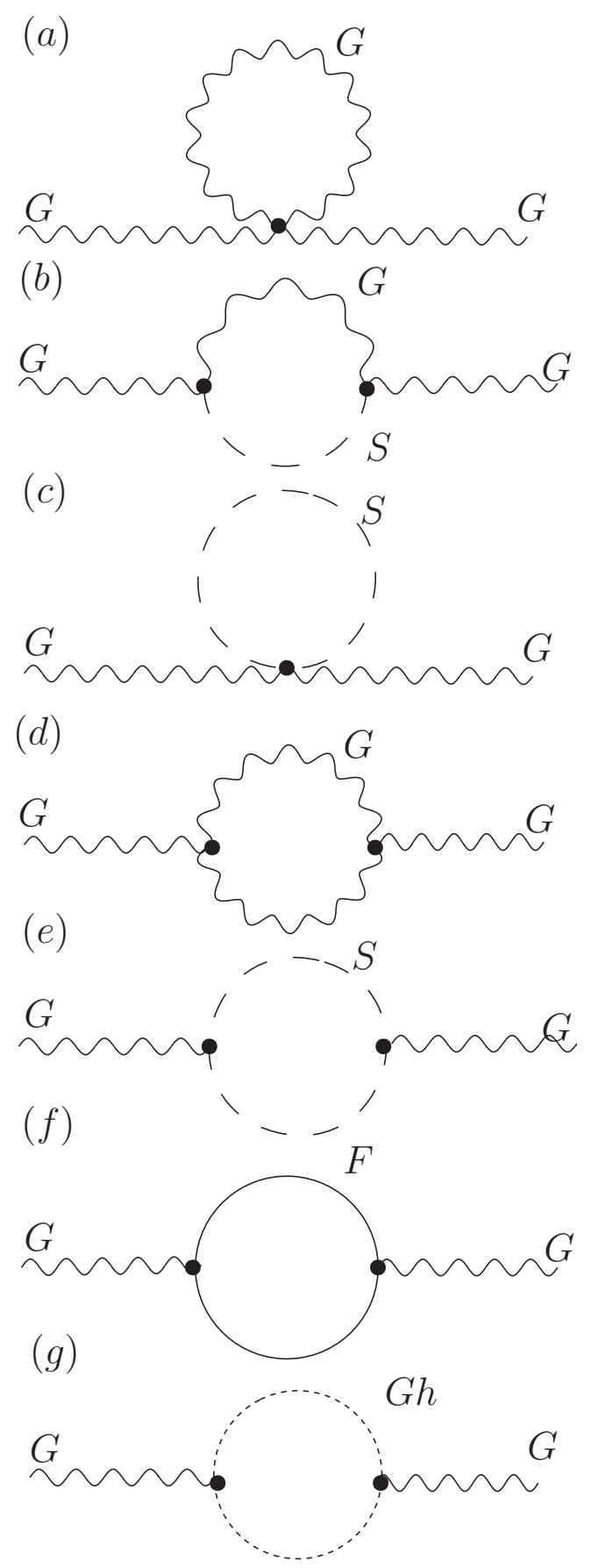

$S$ : Higgs and Goldstone bosons

$G: \gamma, \mathrm{Z}$ and $\mathrm{W}$ gauge bosons

$F$ : fermions

Gh : ghosts

FIG. 2. Feynman diagrams for the Gauge bosons self-energies.

Using the Euclidean version of the gauge boson propagator $G_{B}^{\mu \nu}$ obtained from Eq. (33), and in terms of the 
function $I_{\alpha}$ defined in Eq. (38), we can write the component that is being considered at finite temperature as

$$
\begin{aligned}
\Pi_{a}^{W-W} & =\frac{g^{2}}{3 m_{W}^{2}} T \sum_{n} \int \frac{d^{3} \mathbf{k}}{(2 \pi)^{3}}\left\{9 m_{W}^{2} I_{1}\left(\mathbf{k} ; m_{W}^{2}\right)\right. \\
& +3 \mathbf{k}^{2}\left(I_{1}\left(\mathbf{k} ; m_{W}^{2}\right)-I_{1}\left(\mathbf{k} ; \xi m_{W}^{2}\right)\right) \\
& +(e B)^{2}\left[-3\left(I_{2}\left(\mathbf{k} ; m_{W}^{2}\right)-I_{2}\left(\mathbf{k}, 0 ; \xi m_{W}^{2}\right)\right)\right. \\
& -7 \mathbf{k}^{2}\left(I_{3}\left(\mathbf{k} ; m_{W}^{2}\right)-I_{3}\left(\mathbf{k} ; \xi m_{W}^{2}\right)\right) \\
& +4 \mathbf{k}^{4}\left(I_{4}\left(\mathbf{k} ; m_{W}^{2}\right)-I_{4}\left(\mathbf{k} ; \xi m_{W}^{2}\right)\right) \\
& \left.\left.+15 m_{W}^{2} I_{3}\left(\mathbf{k} ; m_{W}^{2}\right)+12 \mathbf{k}^{2} m_{W}^{2} I_{4}\left(\mathbf{k} ; m_{W}^{2}\right)\right]\right\},
\end{aligned}
$$

where hereafter $\Pi^{00} g b \equiv \Pi^{g b}$ for gauge boson selfenergies.

Using Eqs. (39) and (40) into Eq. (52), including all contributions from gauge bosons in the loop and keeping only the leading terms we get

$$
\begin{aligned}
\Pi_{a}^{W} & =2 g^{2} T^{2}(\xi+1)-\frac{\left(2+\xi^{3 / 2}\right) g^{2} T}{4 \pi\left(g^{2}+g^{\prime 2}\right)} \\
& \times\left(\left(g^{2}+g^{\prime 2}\right) m_{W}+g^{2} m_{Z}+g^{\prime 2} m_{\gamma}\right) \\
& -\frac{g^{2}(e B)^{2}}{128 \pi m_{W}^{2}}\left(\frac{4 T}{3 m_{W}}\left(2-\frac{23}{\xi^{1 / 2}}\right)+\frac{7 \zeta(3) m_{W}^{2}}{\pi^{3} T^{2}}\right) .
\end{aligned}
$$

We now turn to compute the expressions for $\Pi_{b}^{W-Z(\gamma)-S}$ depicted in Fig. 2(b). For the case where the external particle is a $W$-boson and the internal ones are a neutral gauge boson and a charged Goldstone boson, its explicit expression is

$$
\begin{aligned}
\Pi_{b}^{\mu \nu}(q) & =\frac{g^{2} g^{\prime 2}}{g^{2}+g^{\prime 2}}\left\{\begin{array}{c}
\left(m_{Z}^{2}-m_{W}^{2}\right) \\
m_{W}^{2}
\end{array}\right\} \\
& \times \int \frac{d^{4} k}{(2 \pi)^{4}} g_{\mu \rho} G^{\rho \sigma}(k) g_{\nu \sigma} D_{B}(k-q),
\end{aligned}
$$

where the upper (lower) case corresponds to an internal $Z(\gamma)$-line. Notice that since the net charge flowing in the loop is not zero, the phase factor referred to in Eq. (19) does not vanish and should in principle be also included in the computation of this self-energy. Nevertheless, as we are interested in this expression to compute the contribution of the ring diagrams to the EP, that in turn is represented by closed loops, the phase factor becomes the identity and therefore it does not need to be computed for individual self-energies. The same applies for the rest of the self-energy diagrams where there is a net charge flowing in the loop and consequently, we do not consider computing their corresponding phases.

Using the Euclidean version of Eq. (31) and Eq. (33), we have

$$
\begin{aligned}
& \Pi_{b}^{W-Z(\gamma)-S}=-\frac{g^{2} g^{\prime 2}}{g^{2}+g^{\prime 2}} \\
\times & \left\{\begin{array}{c}
\left(m_{Z}^{2}-m_{W}^{2}\right) \\
m_{W}^{2}
\end{array}\right\} T \sum_{n} \int \frac{d^{3} k}{(2 \pi)^{3}} \\
\times & \left\{m_{Z(\gamma)}^{2} I_{11}\left(\mathbf{k}, 0 ; m_{i}^{2}, m_{Z(\gamma)}^{2}\right)\right. \\
+ & \omega_{n}^{2}\left(I_{11}\left(\mathbf{k}, 0 ; m_{i}^{2}, m_{Z(\gamma)}^{2}\right)-I_{11}\left(\mathbf{k}, 0 ; m_{i}^{2}, \xi m_{Z(\gamma)}^{2}\right)\right. \\
+ & \frac{(e B)^{2}}{3}\left[4 \mathbf{k}^{2} m_{Z(\gamma)}^{2} I_{41}\left(\mathbf{k}, 0 ; m_{i}^{2}, m_{Z(\gamma)}^{2}\right)\right. \\
- & 3 m_{Z(\gamma)}^{2} I_{31}\left(\mathbf{k}, 0 ; m_{i}^{2}, m_{Z(\gamma)}^{2}\right) \\
- & 3 \omega_{n}^{2}\left(I_{31}\left(\mathbf{k}, 0 ; m_{i}^{2}, m_{Z(\gamma)}^{2}\right)-I_{31}\left(\mathbf{k}, 0 ; m_{i}^{2}, \xi m_{Z(\gamma)}^{2}\right)\right) \\
+ & \left.4 \omega_{n}^{2} \mathbf{k}^{2}\left(I_{41}\left(\mathbf{k}, 0 ; m_{i}^{2}, m_{Z(\gamma)}^{2}\right)-I_{41}\left(\mathbf{k}, 0 ; m_{i}^{2}, \xi m_{Z(\gamma)}^{2}\right)\right]\right\} .
\end{aligned}
$$

The integrand in Eq. (55) contains terms whose general form is

$$
\begin{aligned}
I_{\alpha \beta}\left(\mathbf{k}, \mathbf{q} ; m^{2}, \xi m^{2}\right) & \equiv \frac{1}{\left[\omega_{n}^{2}+\mathbf{k}^{2}+m^{2}\right]^{\alpha}} \\
& \times \frac{1}{\left[\omega_{n}^{2}+(\mathbf{k}-\mathbf{q})^{2}+\xi m^{2}\right]^{\beta}} .
\end{aligned}
$$

We make use of the Feynman parametrization to write $I_{\alpha \beta}$ as

$$
\begin{aligned}
I_{\alpha \beta}\left(\mathbf{k}, \mathbf{q} ; m^{2}, \xi m^{2}\right) & =\frac{\Gamma(\alpha+\beta)}{\Gamma(\alpha) \Gamma(\beta)} \\
& \times \int_{0}^{1} \frac{d x x^{\alpha-1}(1-x)^{\beta-1}}{\left[\omega_{n}^{2}+\mathbf{k}^{\prime 2}(x)+m^{\prime 2}(x)\right]^{\alpha+\beta}},
\end{aligned}
$$

where

$$
\begin{aligned}
\mathbf{k}^{\prime}(\mathbf{x}) & =\mathbf{k}-(1-x) \mathbf{q} \\
m^{\prime 2}(x) & =m_{W}^{2}(\xi+x-\xi x)+x(1-x) \mathbf{q}^{2},
\end{aligned}
$$

and $\Gamma$ is the Gamma function. Notice that according to our findings in Ref. [41], this parametrization is allowed since we are pursuing a description in the infrared limit. To carry out the sum over Matsubara frequencies together with the integration in Eq. (55), once again we resort to the results in Ref. [40] (see also Ref. 24]). The 
explicit result, generalized to include the fermion case is

$$
\begin{aligned}
T \sum_{n} \int \frac{d^{d} k}{(2 \pi)^{d}} \mathbf{k}^{2 a} \omega_{n}^{2 t} I_{\alpha \beta}\left(\mathbf{k}, \mathbf{q} ; m^{2}, \xi m^{2}\right)=\frac{(2 T)}{(4 \pi)^{d / 2}} \\
\quad \times \frac{(2 \pi T)^{d+2 a+2 t-2(\alpha+\beta)}}{\Gamma(\alpha) \Gamma(\beta)} \frac{\Gamma\left(\frac{d}{2}+a\right)}{\Gamma\left(\frac{d}{2}\right)} \mu^{2 \epsilon} \\
\quad \times \sum_{j=0}^{\infty} \frac{(-1)^{j}}{j !} \zeta\left(2\left(j+\alpha+\beta-t-\frac{d}{2}-a\right), Z\right) \\
\quad \times \Gamma\left(j+\alpha+\beta-\frac{d}{2}-a\right) \\
\quad \times \int_{0}^{1} d x x^{\alpha-1}(1-x)^{\beta-1}\left(\frac{m^{\prime}(x)}{2 \pi T}\right)^{2 j},
\end{aligned}
$$

where for fermions the sum runs over all integers and $Z=1 / 2$. For the terms involving the $n=0$ Matsubara frequency for bosons, we use the result

$$
\begin{aligned}
& T \int \frac{d^{d} k}{(2 \pi)^{d}} \mathbf{k}^{2 a} I_{\alpha \beta}\left(\mathbf{k}, \mathbf{q} ; m^{2}, \xi m^{2}\right)=\frac{T}{(4 \pi)^{d / 2}} \\
& \quad \times \frac{\Gamma\left(\frac{d}{2}+a\right)}{\Gamma\left(\frac{d}{2}\right)} \frac{\Gamma\left(\alpha+\beta-\frac{d}{2}\right)}{\Gamma(\alpha) \Gamma(\beta)} \\
& \quad \times \int_{0}^{1} d x x^{\alpha-1}(1-x)^{\beta-1}\left(\frac{1}{m^{\prime}(x)}\right)^{2 \alpha+2 \beta-d+2 a} .
\end{aligned}
$$

Using Eq. (59) for the terms with $n \neq 0$ and Eq. (60) for $n=0$, and after considering the contributions from all gauge boson fields running in the loop, we get

$$
\begin{aligned}
& \Pi_{b}^{W}=-\frac{\left(g^{2}+g^{\prime 2}\right) m_{W}^{2}}{8 \pi^{2}} \ln \left(\frac{m_{W}}{T}\right)-\frac{T m_{W}^{2}}{4 \pi\left(g^{2}+g^{\prime 2}\right)} \\
& \times\left(\frac{g^{2}\left(g^{2}+g^{\prime 2}\right)}{m_{4}+m_{W}}+\frac{g^{\prime 4}}{m_{1}+m_{Z}}+\frac{g^{2} g^{\prime 2}}{m_{1}+m_{\gamma}}\right) \\
& +(e B)^{2} T\left(-\frac{g^{2} m_{W}^{2}}{48 \pi m_{W}\left(m_{4}+m_{W}\right)^{4}}\left(\frac{m_{4}^{2}}{m_{W}^{2}}+\frac{4 m_{4}}{m_{W}}+1\right)\right. \\
& -\frac{7 g^{2} m_{4} m_{W}^{2}}{32 \pi m_{W}^{2}\left(m_{4}+m_{W}\right)^{3}}\left(\frac{m_{4}}{m_{W}}+3\right) \\
& +\frac{g^{2} m_{W}^{2}}{8 \pi m_{W}^{3}}\left(\frac{1}{\left(m_{4}+m_{W}\right)^{2}}-\frac{1}{\xi^{1 / 2}\left(m_{4}+\xi^{1 / 2} m_{W}\right)^{2}}\right) \\
& -\frac{g^{\prime 4} m_{W}^{2}}{48 \pi m_{1}\left(g^{2}+g^{\prime 2}\right)\left(m_{1}+m_{Z}\right)^{4}}\left(1+\frac{4 m_{Z}}{m_{1}}+\frac{m_{Z}^{2}}{m_{1}^{2}}\right) \\
& +\frac{g^{\prime 4} m_{W}^{2}}{32\left(g^{2}+g^{\prime 2}\right) \pi m_{1}^{2}\left(m_{1}+m_{Z}\right)^{3}}\left(3+\frac{m_{Z}}{m_{1}}\right) \\
& -\frac{g^{2} g^{\prime 2} m_{W}^{2}}{48\left(g^{2}+g^{\prime 2}\right) \pi m_{1}\left(m_{1}+m_{\gamma}\right)^{4}}\left(1+\frac{4 m_{\gamma}}{m_{1}}+\frac{m_{\gamma}^{2}}{m_{1}^{2}}\right) \\
& \left.+\frac{g^{2} g^{\prime 2} m_{W}^{2}}{32\left(g^{2}+g^{\prime 2}\right) \pi m_{1}^{2}\left(m_{1}+m_{\gamma}\right)^{3}}\left(3+\frac{m_{\gamma}}{m_{1}}\right)\right) \\
& +\frac{(e B)^{2} m_{W}^{2} \zeta(5)}{2048 \pi^{6} T^{4}}\left(g^{\prime 4}-15 g^{4}-14 g^{2} g^{\prime 2}\right) \text {. }
\end{aligned}
$$

The rest of the diagrams depicted in Fig. 2] can be computed in the same manner and the result is given in Appendix B. For the expressions corresponding to diagrams $(a)-(g)$ in Fig. 2] some remarks are in order: First, notice that terms of order $m_{g b} / T$, where $m_{g b}$ stands for any of the gauge boson masses, are proportional to $v / T$ and thus, as in the discussion of the scalar self-energy, these terms are small when the position of the minimum in the broken phase is small compared to the critical temperature. Second, when the gauge boson masses appear in the denominators throughout, we should keep in mind that the analysis is valid in the broken phase, where the vacuum expectation value of the Higgs field is different from zero. However, since the analysis considers the effective potential as a function of $v \geq 0$, we will implement this restriction by substituting the gauge boson mass by the thermal ones, when this mass appears in denominators. This is meant to regulate the singularity at $v=0$ and does not make a numerical difference while allowing us to treat the effective potential as a function in the domain $v \geq 0$. The gauge boson thermal masses are defined by

$$
\begin{aligned}
\tilde{m}_{W} & =\sqrt{m_{W}^{2}+\left(\Pi_{W}^{Q}\right)_{1}}, \\
\tilde{m}_{Z} & =\sqrt{m_{Z}^{2}+\left(\Pi_{Z}^{Q}\right)_{1}}, \\
\tilde{m}_{\gamma} & =\sqrt{\left(\Pi_{\gamma}^{Q}\right)_{1}},
\end{aligned}
$$

where $\left(\Pi_{W}^{Q}\right)_{1},\left(\Pi_{Z}^{Q}\right)_{1}$ and $\left(\Pi_{\gamma}^{Q}\right)_{1}$ are the leading terms of the gauge boson self-energies made up by adding the corresponding terms in Appendix B for each gauge boson. These leading terms turn out to be gauge parameter independent and are explicitly given by

$$
\begin{aligned}
& \left(\Pi_{W}^{Q}\right)_{1}=\frac{11}{6} g^{2} T^{2} \\
& \left(\Pi_{Z}^{Q}\right)_{1}=\frac{11}{6} \frac{\left(g^{4}+g^{\prime 4}\right)}{\left(g^{2}+g^{\prime 2}\right)} T^{2}, \\
& \left(\Pi_{\gamma}^{Q}\right)_{1}=\frac{11}{3} \frac{g^{2} g^{\prime 2}}{\left(g^{2}+g^{\prime 2}\right)} T^{2} .
\end{aligned}
$$

As is described in Ref. [33], the other non zero components of the gauge boson self-energy are negligible

$$
\begin{aligned}
\Pi^{11}=\Pi^{22}, \Pi^{33} & \sim \mathcal{O}\left(m_{i}^{2}\right) \\
\Pi^{03}=\Pi^{30} & \sim \mathcal{O}(e B) .
\end{aligned}
$$

From Eqs. (48) and (50), we see that since $\Pi^{M}=-\Pi^{11}$ then $\Pi^{00} \simeq \Pi^{Q}$, and thus

$$
\Pi^{\mu \nu} \simeq \Pi^{00} Q^{\mu \nu} .
$$

\section{EFFECTIVE POTENTIAL}

\section{A. One-loop}

In the standard model the tree level potential is

$$
V_{\text {tree }}(v)=-\frac{1}{2} c^{2} v^{2}+\frac{1}{4} \lambda v^{4} .
$$


To one loop, the EP receives contributions from each sector, namely

$$
V^{(1)}(v)=V_{H}^{(1)}(v)+V_{f}^{(1)}(v)+V_{\mathrm{gb}}^{(1)}(v)+V_{\mathrm{FP}}^{(1)}(v),(67)
$$

where in general each one of these contributions is given by

$$
V^{(1)}(v)=\frac{T}{2} \sum_{n} \int \frac{d^{3} k}{(2 \pi)^{3}} \operatorname{Tr}\left(\ln \left[D\left(\omega_{n}, \mathbf{k}\right)^{-1}\right]\right),
$$

with $D$ standing for either the scalar, fermion, gauge boson or ghost propagator, and the trace is taken over all internal indices. We first discuss the thermal contributions coming from each sector, as described above, and then we implement the renormalization procedure of the temperature independent contributions.

In the weak field limit, the contribution from the Higgs sector is given by

$$
\begin{aligned}
& V_{H}^{(1)}=\sum_{i=1}^{4} \frac{T}{2} \sum_{n} \int \frac{d^{3} k}{(2 \pi)^{3}} \ln \left[D_{B}^{-1}\left(\omega_{n}, \mathbf{k}\right)\right] \\
& \simeq \sum_{i=1}^{4} \frac{T}{2} \sum_{n} \int \frac{d^{3} k}{(2 \pi)^{3}}\left\{\ln \left(\omega_{n}^{2}+\mathbf{k}^{2}+m_{i}^{2}\right)\right. \\
& \left.+(e B)^{2}\left[\frac{1}{\left(\omega_{n}^{2}+\mathbf{k}^{2}+m_{i}^{2}\right)^{2}}-\frac{2\left(k_{\perp}^{2}\right)}{\left(\omega_{n}^{2}+\mathbf{k}^{2}+m_{i}^{2}\right)^{3}}\right]\right\},
\end{aligned}
$$

where the contributions from all scalars have been accounted for.

The first term in Eq. (69) represents the lowest order contribution to the EP at finite temperature and zero external magnetic field, usually referred to as the boson ideal gas contribution [39]. The thermal piece of this contribution is given by [30]

$$
\begin{aligned}
V_{H}^{(1) T \neq 0} & \simeq \sum_{i=1}^{4}\left(-\frac{\pi^{2} T^{4}}{90}+\frac{m_{i}^{2} T^{2}}{24}-\frac{m_{i}^{3} T}{12 \pi}\right. \\
& \left.-\frac{m_{i}^{4}}{32 \pi^{2}} \ln \left(\frac{m_{i}}{4 \pi T}\right)+\mathcal{O}\left(m_{i}^{4}\right)\right) .
\end{aligned}
$$

Notice that there are potentially dangerous terms in Eq. (70) that can become imaginary for negative values of $m_{i}$. However as we will show, these terms cancel when including the Higgs contribution from the vacuum renormalization as well as the ring diagrams.

The second, $B$-dependent term in Eq. (69) vanishes identically 33]. Therefore, to one-loop order, the thermal contribution to the EP in the weak field case from the Higgs sector is independent of $e B$ and is given by Eq. (70).

In the weak field limit, the contribution from the fermion sector is given by

$$
\begin{aligned}
V_{f}^{(1)} & =N_{c} T \sum_{n} \int \frac{d^{3} k}{(2 \pi)^{3}} \ln \left[S_{B}^{-1}\left(\omega_{n}, \mathbf{k} ; m_{t}\right)\right] \\
& \simeq N_{c} 2 T \sum_{n} \int \frac{d^{3} k}{(2 \pi)^{3}}\left\{\ln \left[\omega_{n}^{2}+\mathbf{k}^{2}+m_{t}^{2}\right]\right. \\
& \left.+2(e B)^{2} \frac{\omega_{n}^{2}+k_{3}^{2}+m_{t}^{2}}{\left(\omega_{n}^{2}+\mathbf{k}^{2}+m_{t}^{2}\right)^{3}}\right\}
\end{aligned}
$$

where $N_{c}=3$ is the number of colors. We emphasize that the only fermion mass we keep in the analysis is the top mass $m_{t}$.

The first term in Eq. (71) represents the fermion ideal gas contribution [39], whose thermal part is explicitly given by

$$
\begin{aligned}
V_{f}^{(1) T \neq 0} & \simeq 3\left[-7 \frac{\pi^{2} T^{4}}{180}+\frac{m_{t}^{2} T^{2}}{12}\right. \\
& \left.+\frac{m_{t}^{4}}{16 \pi^{2}} \ln \left(\frac{m_{t}^{2}}{T^{2}}\right)+\mathcal{O}\left(m_{t}^{4}\right)\right]
\end{aligned}
$$

The second term in Eq. (71) is subdominant, after taking care of renormalization as shown in Ref. 24]. Therefore, to one-loop order, the thermal contribution to the EP in the weak field limit from the fermion sector is only given by Eq. (72).

In the gauge boson and ghost sectors, first note that in the broken phase the charged fields do couple to the external magnetic field. Therefore, their contribution to the EP at one-loop contains both magnetic field independent and dependent terms. For the former, the ghost field contributions cancel the spurious degrees of freedom arising in the gauge sector when working in an arbitrary covariant gauge. In the latter, the charged ghost fields contribute as regular scalar fields with the opposite sign and vanishes identically 33] [see discussion after Eq. (70)]. Altogether, the magnetic field dependent contribution from these sectors comes only from the magnetic field dependent pieces of the $\mathrm{W}$ boson propagator.

Therefore, the thermal part of the contribution to the EP from the gauge boson sector is given explicitly by

$$
\begin{gathered}
V_{\mathrm{gb}}^{(1) T \neq 0}+V_{\mathrm{FP}}^{(1) T \neq 0}=-11 \frac{\pi^{2} T^{4}}{90}+3 \frac{\left(2 m_{W}^{2}+m_{Z}^{2}\right) T^{2}}{24}-3 \frac{\left(2 m_{W}^{3}+m_{Z}^{3}\right) T}{12 \pi}-6 \frac{m_{W}^{4}}{32 \pi^{2}} \ln \left(\frac{m_{W}}{4 \pi T}\right)-3 \frac{m_{Z}^{4}}{32 \pi^{2}} \ln \left(\frac{m_{Z}}{4 \pi T}\right) \\
-\frac{(e B)^{2}}{256 \pi^{2}}\left[\mathcal{P}_{0}(\xi)+\mathcal{P}_{1}(\xi) \ln \left(\frac{\tilde{m}_{W}}{T}\right)+\pi \mathcal{P}_{2}(\xi) \frac{T}{\tilde{m}_{W}}+\frac{\zeta(3)}{\pi^{2}} \mathcal{P}_{3}(\xi) \frac{m_{W}^{2}}{T^{2}}+\frac{\zeta(5)}{\pi^{4}} \frac{m_{W}^{4}}{T^{4}}\right]
\end{gathered}
$$


where we have maintained the leading magnetic field dependent term and we defined

$$
\begin{aligned}
\mathcal{P}_{0}(\xi)= & \frac{32(1-5 \xi)}{3 \xi}, \\
\mathcal{P}_{1}(\xi)= & \frac{64(3+7 \xi)}{\xi}, \\
\mathcal{P}_{2}(\xi)= & \frac{8}{3 \xi\left(1+\xi^{1 / 2}\right)}\left(14+53 \xi^{1 / 2}+361 \xi\right. \\
& \left.+170 \xi^{3 / 2}-120 \xi^{2}+\xi^{5 / 2}+\xi^{3}\right) \\
\mathcal{P}_{3}(\xi)= & \frac{8(1+16 \xi)}{3 \xi} .
\end{aligned}
$$

Note that the factors in front of each magnetic field independent contribution in Eq. (73) correspond to the two $W^{\prime}$ s, the $Z$ and the photon polarizations [27]. Also, notice that in the terms that come within the contributions proportional to the magnetic field squared, we have replaced the $W$-boson mass by its thermal one within the argument of the logarithmic function and in the term where that mass appears in the denominator. This is because, as previously discussed, although the analysis is strictly speaking only valid in the broken phase, since we consider the effective potential as a function of $v \geq 0$, to avoid divergences near $v \simeq 0$ we replace the gauge boson mass by the thermal one.

To implement the renormalization procedure, notice that the $T=0$ one-loop EP from Eq. (68) involves the integral

$$
\mathcal{V}(m)=\frac{1}{2} \int \frac{d^{3} k}{(2 \pi)^{3}} \sqrt{k^{2}+m^{2}},
$$

where $m$ stands for the mass of any one of the contributing species. This integral diverges and needs to be regularized. We do so by means of introducing the ultraviolet cutoff $\Lambda$ and thus

$$
\begin{aligned}
\mathcal{V}(m ; \Lambda) & =\frac{1}{4 \pi^{2}} \int_{0}^{\Lambda} k^{2} \sqrt{k^{2}+m^{2}} d k \\
& \stackrel{\Lambda \rightarrow \infty}{\rightarrow} \frac{1}{16 \pi^{2}}\left[\Lambda^{4}+m^{2} \Lambda^{2}+\frac{m^{4}}{8}\right. \\
& \left.+\frac{m^{4}}{2} \ln \left(\frac{m}{2 \Lambda}\right)\right] .
\end{aligned}
$$

Therefore, the $T=0$ part of the one-loop potential can be written as

$$
\begin{aligned}
V^{(1) T=0} & =\mathcal{V}\left(m_{H} ; \Lambda\right)+\sum_{i=1}^{3} \mathcal{V}\left(m_{i} ; \Lambda\right)+8 \mathcal{V}\left(m_{W} ; \Lambda\right) \\
& +4 \mathcal{V}\left(m_{Z} ; \Lambda\right)-12 \mathcal{V}\left(m_{t} ; \Lambda\right)-\sum_{i=1}^{3} \mathcal{V}\left(m_{\eta_{i}} ; \Lambda\right),
\end{aligned}
$$

where the factors in front of each term account for the degrees of freedom of the corresponding species and the

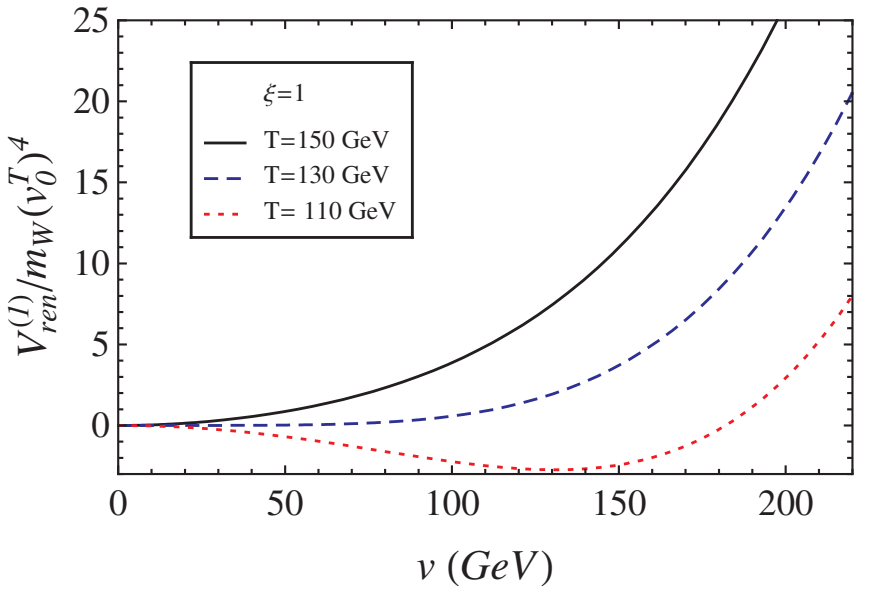

FIG. 3. Renormalized one-loop effective potential for $\xi=1$ and different values of $T$, subtracting $v$ independent terms and dividing by the fourth power of the $v$-dependent $W$ mass evaluated at $v=v_{0}^{T}$, the value of the vacuum expectation value that minimizes de effective potential after the phase transition is completed for a temperature $T=110 \mathrm{GeV}$. In this gauge the phase transition occurs at $T_{c}=128.9 \mathrm{GeV}$ and is second order.

masses depend on $v$. Notice that since we are working in arbitrary covariant gauge the unphysical degrees of freedom for gauge bosons are canceled by the contributions of the ghost fields, which leaves Eq. (77) with only the physical degrees of freedom. We emphasize that the only fermion we consider is the top quark. Since the theory is renormalizable, it should be possible to absorb the $\Lambda$-dependent terms with the introduction of suitable counterterms that maintain the form of the three-level potential. Therefore, the general structure of the $T=0$ effective potential up to one-loop order, after renormalization, should be

$$
\begin{aligned}
V_{\text {ren }}^{(1) T=0}(v) & =-\frac{1}{2} c^{2} v^{2}+\frac{1}{4} \lambda v^{4} \\
& +\frac{a(\Lambda)-\delta c^{2}}{2} v^{2}+\frac{b(\Lambda)+\delta \lambda}{4} v^{4} \\
& +V^{(1) T=0},
\end{aligned}
$$

where the coefficients $a(\Lambda)$ and $b(\Lambda)$ are introduced to cancel the $\Lambda$-dependent terms in $V^{(1) T=0}$ and the coefficients $\delta c^{2}$ and $\delta \lambda$ take care of possible finite corrections of the $v^{2}$ and $v^{4}$ terms, respectively. As the renormalization condition, we require that the minimum of $V_{r e n}^{(1) T=0}(v)$ remains at its classical value, namely

$$
\left.\frac{d V_{\mathrm{ren}}^{(1) T=0}}{d v}\right|_{v=v_{0}}=0 .
$$

Introducing suitable constant terms to make the arguments of the logarithms be dimensionless and disregarding additive constants and terms explicitly proportional 
to $\lambda^{2}$, we get

$$
\begin{aligned}
V_{\text {ren }}^{(1) T=0}(v) & =-\left(1+\frac{3 \lambda}{16 \pi^{2}}+\frac{\left(g^{2}+g^{\prime 2}\right) \xi}{128 \pi^{2}}+2 \frac{g^{2} \xi}{128 \pi^{2}}\right) \\
& \times \frac{c^{2} v^{2}}{2} \\
& +\left(\lambda+\frac{3 f^{4}}{32 \pi^{2}}-\frac{3 g^{4}}{256 \pi^{2}}-\frac{3\left(g^{2}+g^{2}\right)^{2}}{512 \pi^{2}}\right. \\
& \left.-\frac{g^{4} \xi^{2}}{256 \pi^{2}}-\frac{\left(g^{2}+g^{\prime 2}\right)^{2} \xi^{2}}{512 \pi^{2}}\right) \frac{v^{4}}{4} \\
& +\frac{m_{H}^{4}}{64 \pi^{2}} \ln \left(\frac{m_{H}^{2}}{4 c^{2}}\right) \\
& +\sum_{i=1}^{3} \frac{m_{i}^{4}}{64 \pi^{2}} \ln \left(\frac{m_{i}^{2}}{4 c^{2}}\right) \\
& +6 \frac{m_{W}^{4}}{64 \pi^{2}} \ln \left(\frac{m_{W}^{2}}{m_{W}\left(v_{0}\right)^{2}}\right) \\
& +3 \frac{m_{Z}^{4}}{64 \pi^{2}} \ln \left(\frac{m_{Z}^{2}}{m_{Z}\left(v_{0}\right)^{2}}\right) \\
& -12 \frac{m_{f}^{4}}{64 \pi^{2}} \ln \left(\frac{m_{f}^{2}}{m_{f}\left(v_{0}\right)^{2}}\right) .
\end{aligned}
$$

Therefore, after renormalization, the 1-loop EP at finite temperature is given by

$$
\begin{aligned}
V_{\text {ren }}^{(1)}(v) & =-\left(1+\frac{3 \lambda}{16 \pi^{2}}+\frac{\left(g^{2}+g^{\prime 2}\right) \xi}{128 \pi^{2}}+2 \frac{g^{2} \xi}{128 \pi^{2}}\right) \frac{c^{2} v^{2}}{2} \\
& +\left(\lambda+\frac{3 f^{4}}{32 \pi^{2}}-\frac{3 g^{4}}{256 \pi^{2}}\right. \\
& \left.-\frac{3\left(g^{2}+g^{\prime 2}\right)^{2}}{512 \pi^{2}}-\frac{g^{4} \xi^{2}}{256 \pi^{2}}-\frac{\left(g^{2}+g^{\prime 2}\right)^{2} \xi^{2}}{512 \pi^{2}}\right) \frac{v^{4}}{4} \\
& +\sum_{i=1}^{4}\left[\frac{m_{i}^{2} T^{2}}{24}-\frac{m_{i}^{3} T}{12 \pi}+\frac{m_{i}^{4}}{64 \pi^{2}} \ln \left(\frac{(4 \pi T)^{2}}{c^{2}}\right)\right] \\
& +3 \frac{m_{t}^{2} T^{2}}{12}-3 \frac{m_{t}^{4}}{16 \pi^{2}} \ln \left(\frac{T^{2}}{m_{t}^{2}\left(v_{0}\right)}\right) \\
& +3 \frac{\left(2 m_{W}^{2}+m_{Z}^{2}\right) T^{2}}{24}-3 \frac{\left(2 m_{W}^{3}+m_{Z}^{3}\right) T}{12 \pi} \\
& +6 \frac{m_{W}^{4}}{64 \pi^{2}} \ln \left(\frac{(4 \pi T)^{2}}{m_{W}\left(v_{0}\right)^{2}}\right) \\
& +3 \frac{m_{Z}^{4}}{64 \pi^{2}} \ln \left(\frac{(4 \pi T)^{2}}{m_{Z}\left(v_{0}\right)^{2}}\right) \\
& -\frac{(e B)^{2}}{256 \pi} \mathcal{P}_{2}(\xi) \frac{T}{\tilde{m}_{W}},
\end{aligned}
$$

where we have kept only the leading magnetic filed dependent term, with $\mathcal{P}_{2}$ as defined in Eq. (744) and the masses without an argument are the $v$-dependent masses. As pointed out, the dangerous dependence of the scalar masses in the arguments of the logarithmic functions have disappeared. However, to cancel the cubic terms in the scalar masses one needs to go to the next order, namely, to the ring diagrams. Let us for the time being ignore

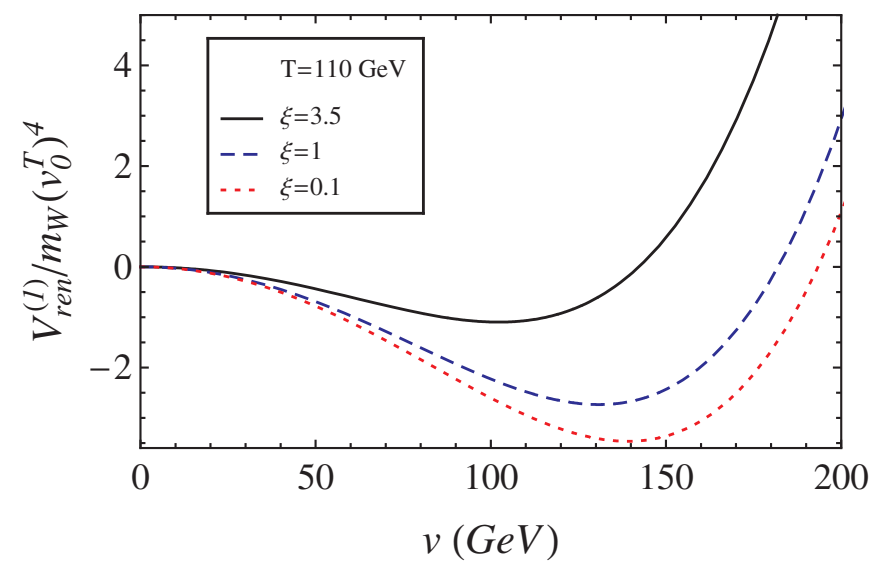

FIG. 4. The renormalized one-loop effective potential normalized by the fourth power of the $v$ dependent $W$-mass evaluated at $v=v_{0}^{T}$, the value of the vacuum expectation value that minimizes de effective potential after the phase transition is completed for a temperature $T=110 \mathrm{GeV}$ and $\xi=1$, as a function of the vacuum expectation value $v$ and for different values of the gauge parameter $\xi$. At this temperature, the position of the minimum $v_{0}$ shows an somewhat significant dependence on the gauge parameter for values $\xi$ around 1 .

the terms proportional to cubic scalar masses and explore the properties of the renormalized effective potential at 1-loop. Figure 3 shows $V_{\text {ren }}^{(1)}(v)$ for $\xi=1$ and different values of $T$, subtracting $v$ independent terms and dividing by the fourth power of the $v$-dependent $W$ mass evaluated at $v=v_{0}^{T}$, the value of the vacuum expectation value that minimizes de effective potential after the phase transition is completed for a temperature $T=110 \mathrm{GeV}$. We can see that the phase transition is second order and in this gauge it happens for $T_{c}=128.9 \mathrm{GeV}$. To illustrate the gauge dependence of the phase transition parameters, Fig. 4 shows $V_{\text {ren }}^{(1)}(v)$ for a low temperature $T=110$ $\mathrm{GeV}$, for which the phase transition has been completed, for different values of the gauge parameter $\xi$. Notice the dependence on the gauge parameter of the broken phase minimum at this constant temperature, whose numerical values are shown on the second column of table I. The dependence on the gauge parameter of the critical temperature is milder, as shown on the first column of table I.

\section{B. Ring diagrams}

It is well known that the next order correction to the EP comes from the so called ring diagrams. These are schematically depicted in Fig. 5. Their renormalized contribution to the EP is more clearly found by explicitly separating the two-loop contribution [39] coming from 


\begin{tabular}{ccc}
\hline$\xi$ & $T_{c}(\mathrm{GeV})$ & $\mathrm{v}_{0}(\mathrm{GeV})$ \\
\hline 3.5 & 120.8 & 102.3 \\
1 & 128.9 & 130.9 \\
0.1 & 132.4 & 139.0 \\
\hline
\end{tabular}

TABLE I. Critical temperature $T_{c}$ (second column) and position of the minimum $\mathrm{v}_{0}$ for the constant temperature $T=110$ $\mathrm{GeV}$ (third column) of the renormalized one-loop effective potential, after the phase transition has been completed. The chosen values of $\xi$ are close to 1, Notice that the critical temperature is only mildly dependent on $\xi$, however the position of the minimum for a temperature where the phase transition has been completed shows a larger $\xi$ dependence.

the scalar sector and can be written as

$$
\begin{aligned}
V_{H}^{(\text {ring })}(v) & \simeq \frac{T}{2} \sum_{n} \int \frac{d^{3} k}{(2 \pi)^{3}}\left\{\left(\ln \left[1+\Pi^{H} D^{H}\right]\right.\right. \\
& \left.-\Pi^{H} D^{H}\right) \\
& +\left(\ln \left[1+\Pi^{G^{0}} D^{G^{0}}\right]-\Pi^{G^{0}} D^{G^{0}}\right) \\
& \left.+2\left(\ln \left[1+\Pi^{G^{c}} D^{G^{c}}\right]-\Pi^{G^{c}} D^{G^{c}}\right)\right\} \\
& +V_{S}^{(2)}
\end{aligned}
$$

where $\Pi^{H}$, $\Pi^{G^{0}}$ and $\Pi^{G^{c}}$ are the Higgs, neutral and charged scalar self-energies in the presence of the magnetic field, $D^{H}, D^{G^{0}}$ and $D^{G^{c}}$ their corresponding propagators and $V_{S}^{(2)}$ the contribution to the two-loop effective potential coming exclusively from the scalar sector. The factor 2 accounts for the two charged scalar degrees of freedom. As written, Eq. (82) deserves some comments: First, the dominant contribution comes from the Matsubara frequency with $n=0$. Second, ultraviolet divergences are canceled explicity. Third, the full two-loop contribution involving the scalar sector contains diagrams with particles other than scalars. For the purposes of the present analysis, we consider only the subclass of diagrams that contain only scalars and thus we get

$$
\begin{aligned}
V_{S}^{(2)} & \simeq \frac{\lambda}{24} T^{4}-\frac{\lambda}{16 \pi}\left(m_{H}+m_{3}+2 m_{1}\right) T^{3} \\
& -(e B)^{2} \frac{\lambda}{192 \pi m_{1}^{3}} T^{3},
\end{aligned}
$$

where we keep only the leading contributions, within the hierarchy of energy scales considered. The potentially dangerous terms with odd powers of the scalar masses in Eq. (83) exactly cancel similar terms coming form the integral in Eq. (82), under the approximation that the self-energies involve only the scalar contribution. The full proof of this cancellation in the absence of magnetic fields has been treated in detail for the standard model in Ref. [28]. In the presence of an external magnetic field, it has been shown in the linear sigma model in Ref. [46]. Within our approximation, this result shows that considering only scalar contributions, this cancellation also

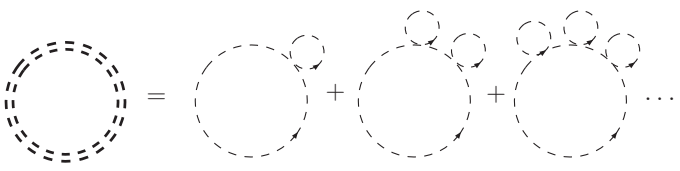

FIG. 5. Schematic representation of the resummation of the ring diagrams.

happens in the standard model with a magnetic field. A full proof is currently under way [47].

The dominant contribution in Eq. (82) comes from the mode $n=0$. The explicit expression for Eq. (82) is [33]

$$
\begin{aligned}
V_{H}^{\text {(ring) }} & =\frac{\lambda}{24} T^{4}-\frac{T}{12 \pi}\left\{\left(m_{H}^{2}+\Pi^{H}\right)^{3 / 2}-m_{H}^{3}\right. \\
& +\left(m_{3}^{2}+\Pi^{G^{0}}\right)^{3 / 2}-m_{3}^{3}+2\left[\left(m_{1}^{2}+\Pi^{G^{c}}\right)^{3 / 2}\right. \\
& \left.\left.-m_{1}^{3}+\frac{(e B)^{2} \Pi_{1}}{16\left(m_{1}^{2}+\Pi^{G^{c}}\right)^{3 / 2}}\right]\right\} .
\end{aligned}
$$

As anticipated, the cubic mass terms in Eq. (84) will exactly cancel the similar terms appearing in Eq. (81) after adding both equations.

Next, we turn to the ring contribution from the gauge boson and Faddeev-Popov ghost sectors. The computation is simplified by recalling that the ghost degrees of freedom cancel the leading temperature contributions from spurious degrees of freedom arising form the covariant treatment of the gauge fields. The contribution that depends on the magnetic field, being subleading, is not canceled, as was the case for the one-loop contribution. Thus we write

$$
\begin{aligned}
V_{\mathrm{gb}}^{(\text {ring })}(v) & +V_{\mathrm{FP}}^{(\text {ring })}=-\frac{T}{2} \sum_{\mathrm{gb}} \sum_{n} \int \frac{d^{3} k}{(2 \pi)^{3}} \\
& \times \operatorname{Tr}\left\{\sum_{N=2}^{\infty} \frac{1}{N}\left[-\Pi_{\mu \lambda \mathrm{gb}}(0) G_{\mathrm{gb}}^{\lambda \nu}\left(\omega_{n}, \mathbf{k}\right)\right]^{N}\right\} \\
& +V_{\text {charged } F P}^{(\text {ring) }}
\end{aligned}
$$

where the sum over gb runs over the four gauge boson physical degrees of freedom and $V_{\text {charged FP }}^{\text {(ring) }}$ represents the subleading, magnetic field dependent term, arising from the ring contribution of the charged ghost fields. Notice that this time, in contrast to the scalar case, we do not add explicitly the two-loop contribution and consequently the sum over the index $N$ starts from $N=2$.

We first compute the contribution from the neutral gauge bosons to Eq. (85). In the approximation where the particle has a small momentum (the infrared limit), the Euclidean version of a neutral, gauge boson propagator, for an arbitrary value of the gauge parameter $\xi$ can be written as

$$
G_{\mu \nu}=\frac{1}{\left(k^{2}+m_{\mathrm{gb}}^{2}\right)}\left\{P_{\mu \nu}^{L}+P_{\mu \nu}^{T}+\xi \frac{k_{\mu} k \nu}{\left(k^{2}+\xi m_{\mathrm{gb}}^{2}\right)}\right\},
$$


where

$$
\begin{gathered}
P_{00}^{T}=P_{0 i}^{T}=0 \quad P_{i j}^{T}=\delta_{i j}-\hat{\mathbf{k}}_{i} \hat{\mathbf{k}}_{j} \\
P_{\mu \nu}^{L}=\delta^{\mu \nu}-\frac{k_{\mu} k_{\nu}}{k^{2}}-P_{\mu \nu}^{T} .
\end{gathered}
$$

By using Eqs. (65) and (87), it is easy to see that the product $\Pi_{\mu \lambda}(0) G^{\lambda \nu}\left(\omega_{n}, \mathbf{k}\right)$ becomes

$$
\Pi_{\mu_{\lambda}} G^{\lambda \nu}=\frac{\left(\Pi_{\mathrm{gb}}^{Q}\right)_{1}}{\left(k^{2}+m_{\mathrm{gb}}^{2}\right)}\left[1+\xi \frac{(k \cdot u)^{2}}{\left(k^{2}+\xi m_{\mathrm{gb}}^{2}\right)}\right] Q_{\mu}^{\nu},
$$

from where, considering the $n=0$ term and taking the trace, we can write Eq. 850 as

$$
\begin{aligned}
V_{\text {neutral gb }}^{(\text {ring }}(v) & \simeq \sum_{\mathrm{gb}} \frac{1}{2} T \int \frac{d^{3} k}{(2 \pi)^{3}}\left[\ln \left(1+\frac{\left(\Pi_{\mathrm{gb}}^{Q}\right)_{1}}{\mathbf{k}^{2}+m_{\mathrm{gb}}^{2}}\right)\right. \\
& \left.-\frac{\left(\Pi_{\mathrm{gb}}^{Q}\right)_{1}}{\mathbf{k}^{2}+m_{\mathrm{gb}}^{2}}\right] \\
& =-\frac{T}{12 \pi}\left\{\tilde{m}_{Z}^{3}-m_{Z}^{3}+\tilde{m}_{\gamma}^{3}\right\} .
\end{aligned}
$$

Notice that the plasma screening effects in Eq. 889) are naturally accounted for by the fact that the thermal modification of the gauge boson mass $\tilde{m}_{\mathrm{gb}}\left(\tilde{m}_{Z}\right.$ and $\left.\tilde{m}_{\gamma}\right)$ appear. Also, the nice cancellations that took place in the scalar sector between odd powers of the masses will not happen in this case when adding these terms to Eq. (81).
This is because in the infrared limit there is no contribution from the gauge boson transverse degrees of freedom, thus there is no match of the coefficients to produce the cancellation. This is a feature of the infrared limit we are considering. However, since the square of the gauge boson masses are never negative, these terms do not pose a problem.

Next, we turn to the contribution of the charged gauge fields. In the weak field limit, the product $\Pi_{\mu \lambda}(0) G^{\lambda \nu}\left(\omega_{n}, \mathbf{k}\right)$ can be written as

$$
\begin{aligned}
\Pi_{\mu \lambda} G^{\lambda \nu}= & \frac{\left(\Pi_{W}^{Q}\right)_{1}}{k^{2}+m_{W}^{2}}\left[1+(e B)^{2}\left(\frac{1}{\left(k^{2}+m^{2}\right)^{2}}\right.\right. \\
& \left.\left.-\frac{2 k_{\perp}^{2}}{\left(k^{2}+m^{2}\right)^{3}}\right)\right] Q_{\mu}^{\nu},
\end{aligned}
$$

Using Eq. (90), and carrying out an expansion of the argument of the logarithm, we can explicitly write

$$
\begin{aligned}
\ln \left[1+\Pi_{\mu \lambda} G^{\lambda \nu}\right] & =\ln \left\{1+\left[\frac{\left(\Pi_{W}^{Q}\right)_{1}}{\mathbf{k}^{2}+m_{W}^{2}}\right] Q_{\mu}^{\nu}\right\} \\
& +\ln \left\{1+\left[\frac{\left(\Pi_{W}^{Q}\right)_{1}}{\mathbf{k}^{2}+m_{W}^{2}+\left(\Pi_{W}^{Q}\right)_{1}}\right.\right. \\
& \times\left(\frac{1}{\left(\mathbf{k}^{2}+m_{W}^{2}\right)^{2}}-\frac{2 k_{\perp}^{2}}{\left(\mathbf{k}^{2}+m_{W}^{3}\right)^{3}}\right) \\
& \left.\left.\times(e B)^{2}\right] Q_{\mu}^{\nu}\right\} .
\end{aligned}
$$

Using this result into Eq. (85), considering the $n=0$ term and taking the trace in Eq. (85) we get

$$
\begin{aligned}
V_{\text {charged gb }}^{(\text {ring }}(v) & =\sum_{\mathrm{gb}} \frac{1}{2} T \int \frac{d^{3} k}{(2 \pi)^{3}}\left\{\left[\ln \left(1+\frac{\left(\Pi_{W}^{Q}\right)_{1}}{\mathbf{k}^{2}+m_{\mathrm{gb}}^{2}}\right)-\frac{\left(\Pi_{W}^{Q}\right)_{1}}{\mathbf{k}^{2}+m_{\mathrm{gb}}^{2}}\right]\right. \\
& \left.+\ln \left[1+\frac{\left(\Pi_{W}^{Q}\right)_{1}}{\left(\mathbf{k}^{2}+m_{g b}^{2}+\left(\Pi_{W}^{Q}\right)_{1}\right)}\left(\frac{1}{\left(\mathbf{k}^{2}+m_{\mathrm{gb}}^{2}+\left(\Pi_{W}^{Q}\right)_{1}\right)^{2}}-\frac{2 k_{\perp}^{2}}{\left(\mathbf{k}^{2}+m_{\mathrm{gb}}^{2}+\left(\Pi_{W}^{Q}\right)_{1}\right)^{3}}\right)(e B)^{2}\right]\right\} \\
& =-2 \frac{T}{12 \pi}\left(\tilde{m}_{W}^{3}-m_{W}^{3}\right)-\frac{(e B)^{2}}{4 \pi}\left(\frac{\left(\Pi_{W}^{Q}\right)_{1}}{48}\right)\left(\frac{T}{\tilde{m}_{W}^{3}}\right) .
\end{aligned}
$$

In this approximation, the plasma screening effects in Eq. (92) emerge naturally as a thermal modification of the gauge boson mass, $\tilde{m}_{W}$ defined in Eqs. (62) and (63).

Finally, the magnetic field dependent contribution from the charged ghost fields to the ring potential is easily computed recalling that this contribution is equivalent to the one coming from two scalars (one for each $W$ field) but with opposite sign. Thus we have

$$
V_{\text {charged FP }}^{\text {(ring) }}=2 \frac{(e B)^{2}}{2 \pi}\left(T \frac{\left(\Pi_{\eta_{W}}\right)_{1}}{48\left(\tilde{m}_{\eta_{W}}\right)^{3}}\right),
$$

where the diagram depicting the Faddeev-Popov ghosts is shown in Fig. 6 and, as shown in Appendix C, the leading contribution to the $W$-ghost self energy is given by

$$
\begin{aligned}
\left(\Pi_{\eta_{W}}\right)_{1}= & \frac{\xi^{2} T m_{W} \mathrm{~g}^{2}}{16 \pi} \times \\
& \left\{\frac{m_{W}}{\tilde{m}_{3}+\xi^{1 / 2} m_{W}}-\frac{m_{W}}{\tilde{m}_{4}+\xi^{1 / 2} m_{W}}\right. \\
+ & \left.\frac{\mathrm{g}^{2}-\mathrm{g}^{\prime 2}}{\mathrm{~g}\left(\mathrm{~g}^{2}+\mathrm{g}^{\prime 2}\right)^{1 / 2}}\left(\frac{m_{Z}}{\tilde{m}_{1}+\sqrt{\xi} m_{Z}}\right)\right\},
\end{aligned}
$$

and the thermal $W(Z)$-ghost mass is given by

$$
\tilde{m}_{\eta_{W(Z)}}=\sqrt{\xi m_{W(Z)}^{2}+\left(\Pi_{\left.\eta_{W(Z)}\right)},\right.},
$$




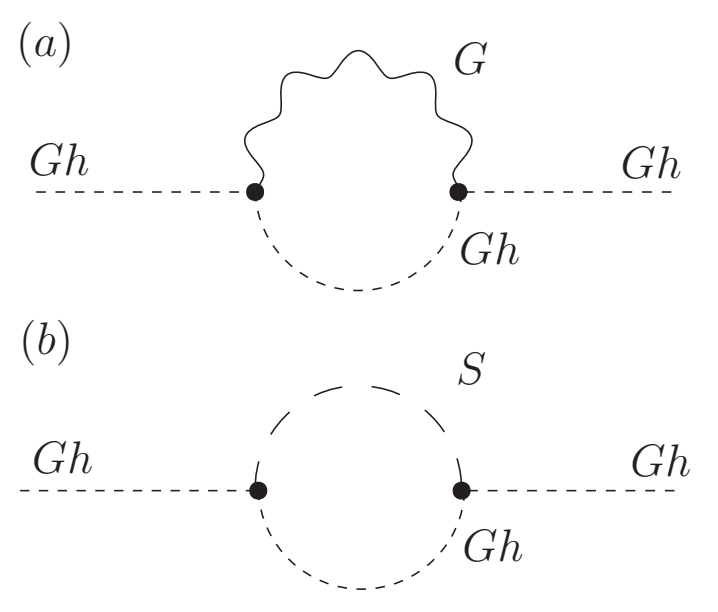

$S:$ Higgs and Goldstone bosons

$G: \gamma, \mathrm{Z}$ and $\mathrm{W}$ gauge bosons

Gh: ghosts

FIG. 6. Feynman diagrams for the Faddeev-Popov ghosts self-energies. Notice that since we work in the infrared limit, the diagrams of type (a), do not contribute to the ghosts selfenergies.

$m_{W}$ and $m_{Z}$ are the $v$-dependent $W$ and $Z$ masses, respectively and $\tilde{m}_{i}(i=1 \ldots 4)$ are defined in Eqs. (4) and (47). Notice that in Eq. (94), we have replaced $m_{i}$ by $\tilde{m}_{i}$ since, although the analysis is valid near the broken phase minimum, we consider the effective potential as a function of $v \geq 0$, and for small values of $\mathrm{v}$, the square of the scalar masses can become negative.

\section{Effective potential up to ring order}

The final expression for the effective potential

$$
\begin{aligned}
V_{\mathrm{eff}}(v) & =V_{\text {tree }}(v)+V_{H}^{(1)}+V_{f}^{(1)}+V_{\mathrm{gb}}^{(1)}+V_{\mathrm{FP}}^{(1)} \\
& +V_{H}^{(\text {ring })}+V_{\mathrm{gb}}^{(\text {ring })}+V_{F P}^{(\text {ring })}
\end{aligned}
$$

is obtained by adding up the results in Eqs. (66), (70), (72), (73), (84), (92) and (93).

In order for the terms involving the square of the scalar bosons' thermal mass to be real, the temperature must be such that

$$
T>T_{1} \equiv \sqrt{\frac{16 c^{2}}{3 \mathrm{~g}^{2}+\mathrm{g}^{2}+8 \lambda+4 f^{2}}},
$$

which defines a lower bound for the temperature. A more restrictive bound is obtained by requiring that $e B<\tilde{m}_{H}^{2}$ for the weak field expansion to work. This condition translates into the bound

$$
T>T_{2} \equiv \sqrt{\frac{e B+16 c^{2}}{3 \mathrm{~g}^{2}+\mathrm{g}^{\prime 2}+8 \lambda+4 f^{2}}} .
$$

The relevant factor that enhances the order of transition, present both in $V_{H}^{(\text {ring) }}$ and $V_{\mathrm{gb}}^{(\text {ring })}$, is $(e B)^{2} / \tilde{m}_{i}^{3}$ which can be traced back to the boson self-energy diagrams involving a tadpole of charged scalars in the presence of the external field.

\section{SYMMETRY RESTORATION}

In order to quantitatively check the effect of the magnetic field during the EWPT, we proceed to plot $V_{\text {eff }}$ as a function of the vacuum expectation value $v$. For the analysis we use $\mathrm{g}^{\prime}=0.344$ and $\mathrm{g}=0.637, m_{Z}=91 \mathrm{GeV}$, $m_{W}=80 \mathrm{GeV}, f=1, \lambda=0.11$ which corresponds to the current bound on the Higgs mass.

Figure 7 shows the effective potential in the absence of magnetic field divided by $\left[m_{W}\left(v_{1}^{T}\right)\right]^{4}=(10.5 \mathrm{GeV})^{4}$, where $v_{1}^{T}$ is the value where the broken phase minimum appears at the critical temperature, which in this case happens to be $T=139.758 \mathrm{GeV}$. The value of the gauge parameter is $\xi=0.1$. The phase transition is weakly first order. Note that the results are consistent with the ones obtained in Ref. 24] which are computed using symmetric phase degrees of freedom.

Figure 8 shows the effective potential divided by $\left[m_{W}\left(v_{1}^{T}\right)\right]^{4}=(10.5 \mathrm{GeV})^{4}$ for the same temperatures as in Fig. 7 and a fixed value of the magnetic field parametrized as $B=b \times(100 \mathrm{GeV})^{2}$, with $b=0.01$. An insert in this plot shows the difference $\left(\Delta V_{\text {eff }}\right)$ with respect to the effective potential shown in Fig. 7 in the absence of magnetic field over a small region in the $\nu$ range where the second minimum would be developing and is now delayed by the presence of magnetic field. This same effect can be observed if we keep the temperature fixed and increase the value of the magnetic field. Given that we use a weak magnetic field, the effect is small. In order to appreciate such effect, in Fig. 9. we show the difference between the effective potentials in the presence and in the absence of magnetic field. Starting from zero magnetic field, for which the phase transition happens at the critical temperature $T=139.758 \mathrm{GeV}$, the phase transition is delayed by increasing the values of the magnetic field, also parametrized as $B=b \times(100 \mathrm{GeV})^{2}$, while the temperature is maintained fixed. In both Figs. 8 and 9 , the value of the gauge parameter is $\xi=0.1$.

Figure 10 shows a small region around the second minima that develops in the effective potential divided by the fourth power of the $v$-dependent $W$ mass evaluated at $v_{1}^{T}$, for three different values of the magnetic field and computed at their corresponding critical temperatures, keeping $\xi=0.1$. Notice that for increasing values of the magnetic field, the phase transition starts at a lower critical temperature $T_{c}$ and the broken phase minimum $v_{0}$ is 


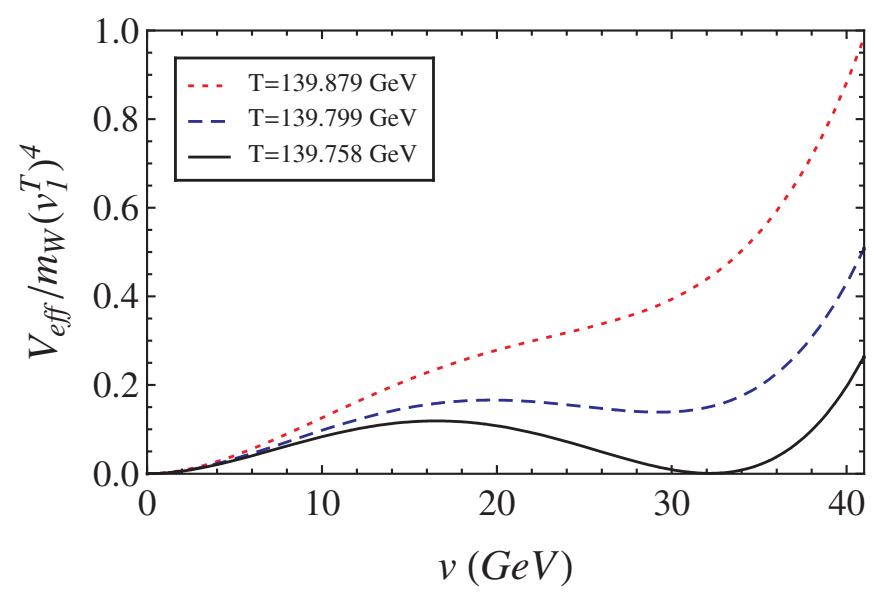

FIG. 7. Effective potential divided by the fourth power of the $v$-dependent $W$ mass evaluated at $v_{1}^{T}$ (the value where the broken phase minimum appears at the critical temperature in the absence of magnetic field). The gauge parameter $\xi=0.1$ and the value of the magnetic field is set to zero. The phase transition is weakly first order. Note that the results are consistent with the ones obtained by [24] which are computed using symmetric phase degrees of freedom

also shifted to higher values in such a way that the ratio $v_{0} / T_{c}$ increases at the phase transition. This increase, although modest, is a desired feature that can eventually

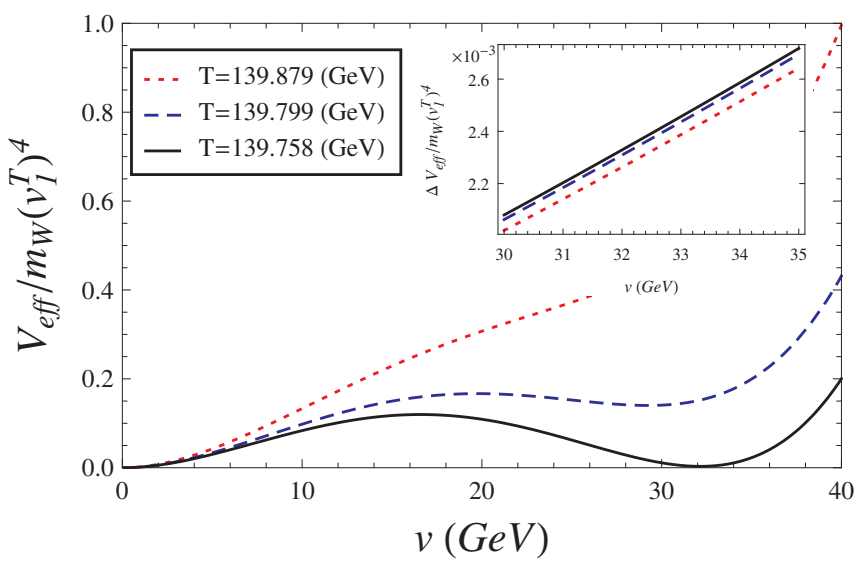

FIG. 8. Effective potential divided by the fourth power of the $v$-dependent $W$ mass evaluated at $v_{1}^{T}$ (the value where the broken phase minimum appears at the critical temperature in the absence of magnetic field) for the same temperatures as in Fig. 7 and a fixed value of the magnetic field parametrized as $B=b \times(100 \mathrm{GeV})^{2}$, with $b=0.01$. The gauge parameter is $\xi=0.1$. The insert shows the difference $\left(\Delta V_{\text {eff }}\right)$ with respect to the effective potential shown in Fig. 7 in the absence of magnetic field over a small region in the $\nu$ range where the second minimum would be developing and is now delayed by the presence of magnetic field.

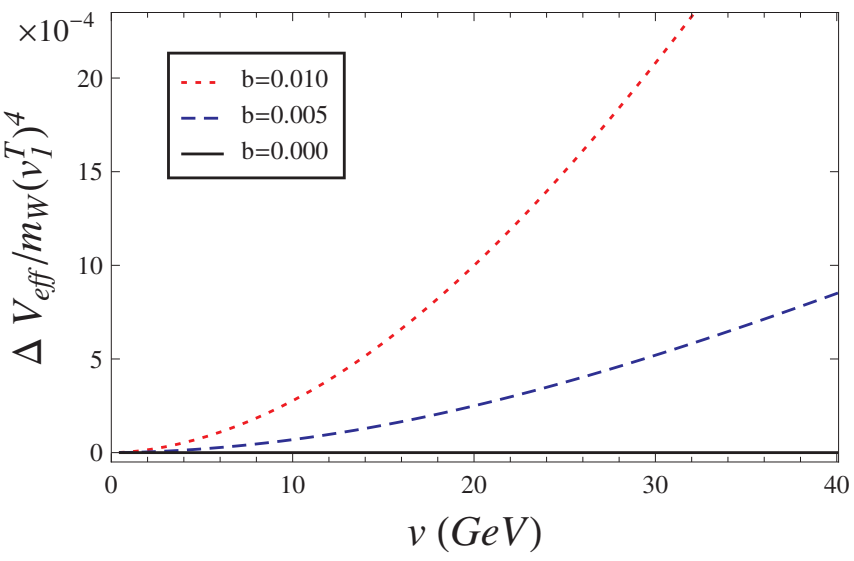

FIG. 9. Difference between the effective potentials in the presence and in the absence of magnetic field divided by the fourth power of the $v$-dependent $W$ mass evaluated at $v_{1}^{T}$ (the value where the broken phase minimum appears at the critical temperature $T=T_{c}^{B=0}=139.758 \mathrm{GeV}$ in the absence of magnetic field) for different values of the magnetic field parametrized as $B=b \times(100 \mathrm{GeV})^{2}$. The gauge parameter is $\xi=0.1$. Even though the magnetic field is weak, we can appreciate that the phase transition is delayed when increasing the values of the magnetic field.

help a possible baryon asymmetry to not be washed out after the completion of the phase transition [3].

To explore the gauge parameter dependence of the effective potential's relevant parameters, Figs. 11- 13] show the difference in the behavior with and without magnetic

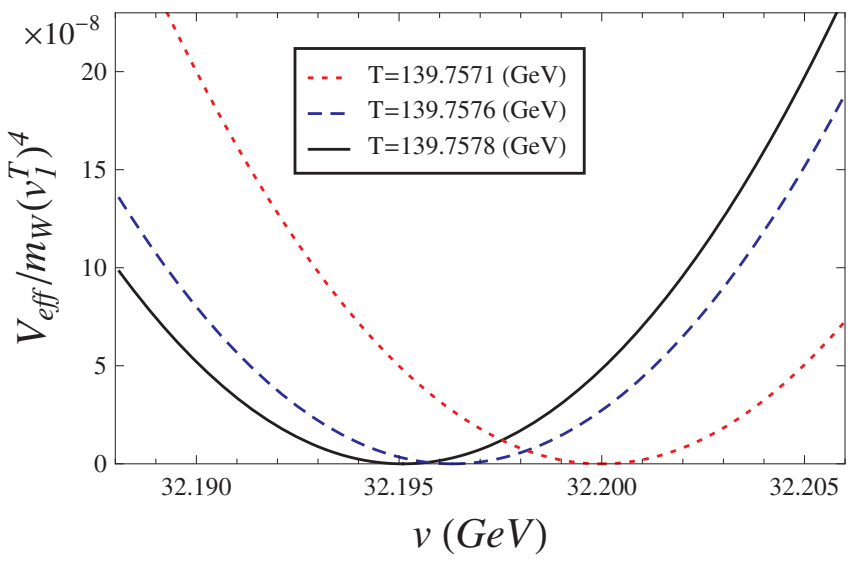

FIG. 10. A small region around the second minima that develops in the effective potential divided by the fourth power of the $v$-dependent $W$ mass evaluated at $v_{1}^{T}$ (the value where the broken phase minimum appears at the critical temperature in the absence of magnetic field) for three different values of the magnetic field at their corresponding critical temperatures and for $\xi=0.1$. Notice how for increasing values of the magnetic field, the phase transition starts at a lower temperature and the broken phase minimum is also shifted to higher values in such a way that the ratio $v_{0} / T_{c}$ increases at the phase transition. 


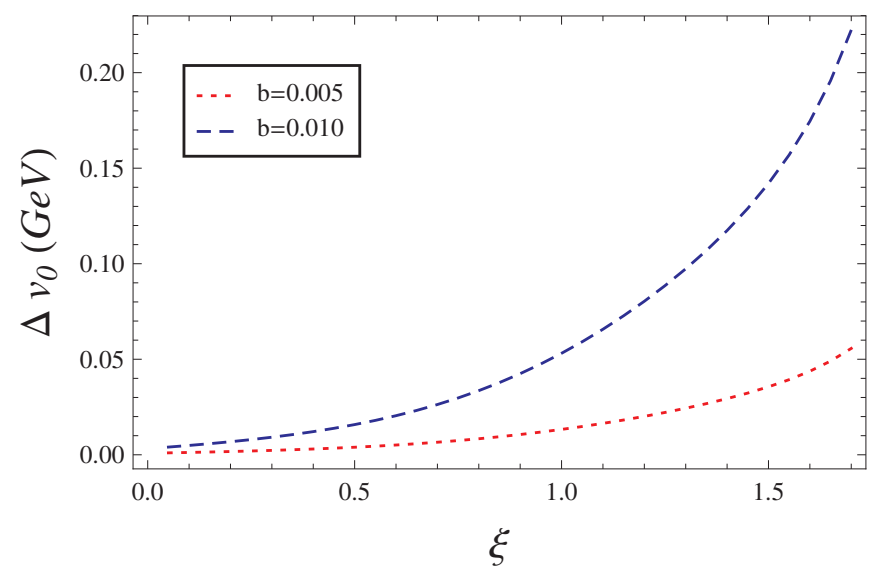

FIG. 11. Difference of gauge parameter dependence of $v_{0}$ with and without magnetic field. The position of the broken phase minimum at the critical temperature, for two values of the magnetic field parametrized as $B=b \times(100 \mathrm{GeV})^{2}$ remains stable throughout a large range of $\xi$, even in the presence of magnetic field.

field of $v_{0}, T_{c}$ and the ratio $v_{0} / T_{c}$ for values of $\xi$ around 1 . We use a fixed value of the magnetic field, parametrized as $B=b \times(100 \mathrm{GeV})^{2}$, with $b=0,0.005,0.01$. We can see that $v_{0}, T_{c}$ and $v_{0} / T_{c}$ remain stable for a variation of $\xi$ up to 1.5. For these small values of the magnetic field strenght, consistent with the assumed hierarchy of energy scales, the magnetic field does not introduce a strong gauge dependence.

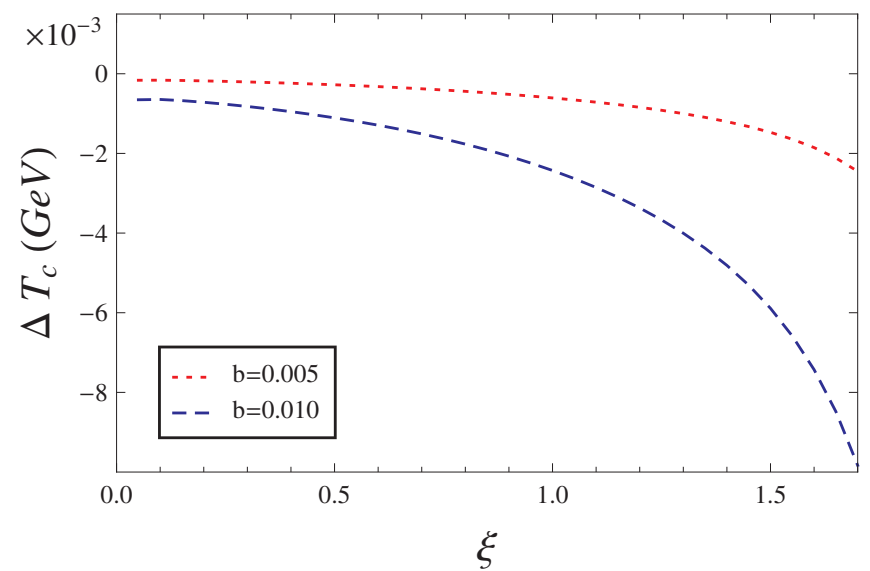

FIG. 12. Difference of gauge parameter dependence of $T_{c}$ with and without magnetic field. The position of the critical temperature for the development of the broken phase minimum, for two values of the magnetic field parametrized as $B=b \times(100 \mathrm{GeV})^{2}$ remains stable throughout a large range of $\xi$.

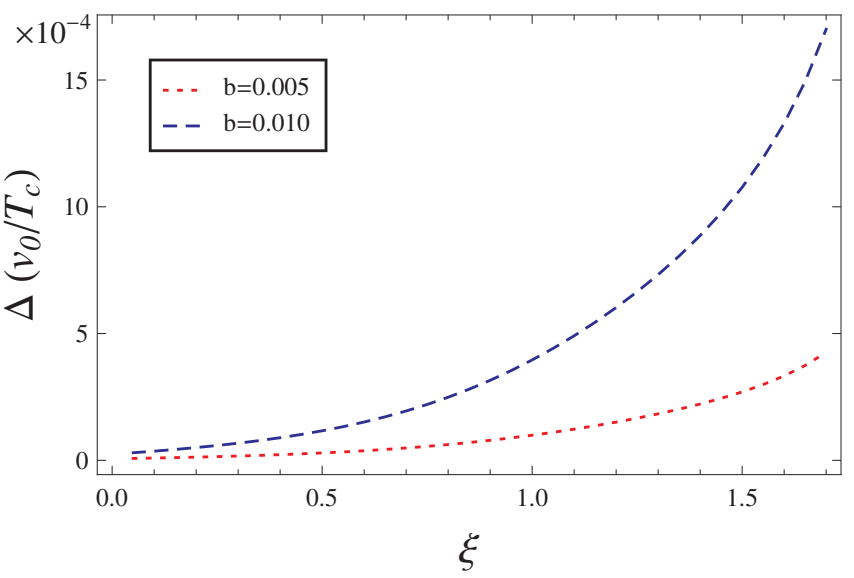

FIG. 13. Difference of gauge parameter dependence of the ratio $v_{0} / T_{c}$ with and without magnetic field, for two values of the magnetic field parametrized as $B=b \times(100 \mathrm{GeV})^{2}$. Notice how the value of $v_{0} / T_{c}$ is stable for a variation of $\xi$ up to 1.5. The presence of the magnetic field does not introduce a strong gauge dependence.

\section{DISCUSSION AND CONCLUSIONS}

In conclusion, we have studied the symmetry restoration problem in the MSM at finite temperature in the presence of an external magnetic field. The study has been carried out by analyzing the finite temperature effective potential. We have shown that in the presence of the magnetic field, the EWPT has become a stronger first order phase transition. Our treatment has been implemented for the case of weak magnetic fields for the hierarchy of scales $e B \ll m^{2} \ll T^{2}$ where $m$ is taken as a generic mass involved in the calculation.

We have explicitly worked with the degrees of freedom in the broken symmetry phase where the external magnetic field belongs to the $U(1)_{e m}$ group and in a covariant gauge, with an arbitrary value of a single gauge fixing parameter. The calculation is carried out up to the contribution of ring diagrams. To include the effects of the magnetic field, we have made use of the Schwinger proper-time method to describe the particle's propagators. In this way, the contribution from all Landau levels has been accounted for. We have carried out a systematic expansion up to order $(e B)^{2}$ and have computed, as an intermediate step, the magnetic field and gauge fixing parameter dependent self-energies in the MSM [48].

The presence of the external magnetic field gives rise to terms in the effective potential proportional to $1 / \tilde{m}_{i}^{3}$, where $\tilde{m}_{i}^{2}=m_{i}^{2}+\Pi_{1}$, coming from tadpole diagrams in the boson self-energies where the loop particle is a charged scalar and $m_{i}$ are their masses. These terms are the relevant ones for the strengthening of the order of the phase transition. The results are in qualitative and quantitative agreement with the ones previously found 24] using symmetry restored degrees of freedom. The ratio of 
the broken phase vacuum expectation value to the critical temperature at the phase transition increases with increasing values of the magnetic field. This feature works in favor of the suppression of the sphaleron induced transitions in the broken phase after the EWPT is completed and thus against a washing out of a possibly produced baryon number. The increase in this ratio is modest, though it is important to notice that we have used very restrictive values of the field strength, consistent with the working hierarchy of energy scales used in this work. The leading temperature terms of the effective potential are gauge parameter independent, although the subleading ones, i.e., the ones coming with the magnetic field turn out to be gauge parameter dependent. The dependence on this parameter of phase transition observables such as the position of the minimum in the symmetry broken phase and the critical temperature for the transition is not negligible for values of $\xi$ near $\xi \sim 1$. This dependence signals that the truncation of the magnetic field power series might not be a gauge invariant procedure. This point deserves a closer look and is being investigated [47].

\section{ACKNOWLEDGMENTS}

Support has been received in part by DGAPA-UNAM under PAPIIT Grants Nos. IN116008 and IN112308 and by CONACyT-México under Grant 52547-F. J. Navarro acknowledges support received by Universidad del Atlántico. M.E.T.-Y. thanks the Programa de Intercambio UNAM-UNISON for support. A.A. and J.N. thank the support provided by CONACyT-México under Grant 40025-f.

\section{APPENDIX A: SCALAR SELF-ENERGIES IN ARBITRARY GAUGE}

In this appendix, we list the results for the self-energy diagrams depicted in Fig. 10 (for the neutral Goldstone boson there is no contribution of the type shown in diagram (c) of Fig. 1).

\section{A. Higgs boson $(H)$ self-energy in arbitrary gauge}

$$
\begin{aligned}
\Pi_{a}^{H}= & \lambda \frac{T^{2}}{2}\left[1-\frac{1}{2 \pi T}\left(2 m_{1}+m_{3}+3 m_{4}\right)\right]-\lambda \frac{(e B)^{2}}{48 \pi m_{1}^{2}}\left[\frac{T}{m_{1}}+\frac{\zeta(3) m_{1}^{2}}{4 \pi^{3} T^{2}}\right] \\
\Pi_{b}^{H}= & \frac{T^{2}}{16}\left[g^{2}(3+\xi)+g^{\prime 2}\left(1+\frac{\xi}{3}\right)-\frac{3+\xi^{3 / 2}}{\pi T}\left(2 g^{2} m_{W}+\left(g^{2}+g^{\prime 2}\right) m_{Z}\right)\right] \\
& +\frac{(e B)^{2} g^{2}}{64 \pi m_{W}^{2}}\left[\left(3+\frac{35}{3 \xi^{1 / 2}}\right) \frac{T}{m_{W}}+\frac{11 \zeta(3) m_{W}^{2}}{3 \pi^{3} T^{2}}\right] \\
\Pi_{c}^{H}= & -\frac{\left(3 g^{4}+2 g^{2} g^{\prime 2}+g^{\prime 4}\right) m_{W}^{2}}{4 g^{2} \pi^{2}} \ln \left(\frac{m_{W}}{T}\right) \\
& -\frac{T}{2 \pi}\left[g^{2} m_{W}+\frac{g^{2} m_{W}^{2}}{2 m_{Z}}+\frac{g^{\prime 2} m_{W}^{2}}{m_{Z}}+\frac{g^{\prime 4} m_{W}^{2}}{2 g^{2} m_{Z}}\right]\left(\frac{3}{4}+\frac{5 \xi^{3 / 2}}{4}-\frac{\xi^{3 / 2}}{\left(1+\xi^{1 / 2}\right)}-\frac{\xi^{2}}{\left(1+\xi^{1 / 2}\right)}\right) \\
& +(e B)^{2} \frac{g^{2} T}{\pi m_{W}^{3}}\left(-\frac{83}{192}+\frac{13}{12\left(1+\xi^{1 / 2}\right)^{4}}+\frac{5}{48\left(1+\xi^{1 / 2}\right)^{3}}\right. \\
& -\frac{5}{4\left(1+\xi^{1 / 2}\right)^{2}}-\frac{1}{192 \xi^{1 / 2}}+\frac{7}{24\left(1+\xi^{1 / 2}\right)^{4} \xi^{1 / 2}}-\frac{7}{16\left(1+\xi^{1 / 2}\right)^{3} \xi^{1 / 2}} \\
& -\frac{1}{2\left(1+\xi^{1 / 2}\right)^{2} \xi^{1 / 2}}+\frac{9 \xi^{1 / 2}}{4\left(1+\xi^{1 / 2}\right)^{4}}+\frac{3 \xi^{1 / 2}}{16\left(1+\xi^{1 / 2}\right)^{3}}-\frac{3 \xi^{1 / 2}}{4\left(1+\xi^{1 / 2}\right)^{2}} \\
& \left.+\frac{9 \xi}{4\left(1+\xi^{1 / 2}\right)^{4}}-\frac{17 \xi}{48\left(1+\xi^{1 / 2}\right)^{3}}+\frac{7 \xi^{3 / 2}}{8\left(1+\xi^{1 / 2}\right)^{4}}+\frac{1}{4\left(1+\xi^{1 / 2}\right)}\right)-\frac{9(e B)^{2} g^{2} m_{W}^{2} \zeta(5)}{256 \pi^{6} T^{4}}
\end{aligned}
$$




$$
\begin{aligned}
\Pi_{d}^{H}= & -T^{2} \frac{\left(3 g^{2}+g^{\prime 2}\right) \xi}{48} \\
& +T\left(\frac{g^{2} m_{1}^{3}}{8 \pi m_{W}\left(m_{1}+m_{W}\right)}\left(\frac{m_{1}^{2}\left(m_{1}+m_{W}\right)}{m_{W}\left(m_{1}^{2}-\xi m_{W}^{2}\right)}-\frac{\xi^{5 / 2} m_{W}^{4}\left(m_{1}+m_{W}\right)}{m_{1}^{3}\left(m_{1}^{2}-\xi m_{W}^{2}\right)}-\frac{m_{1}}{m_{W}}-1\right)\right. \\
& +\left(g^{2}+g^{\prime 2}\right) \frac{m_{3}^{3}}{16 \pi m_{Z}\left(m_{3}+m_{Z}\right)}\left(\frac{m_{3}^{2}\left(m_{3}+m_{Z}\right)}{m_{Z}\left(m_{3}^{2}-\xi m_{Z}^{2}\right)}-\frac{\xi^{5 / 2} m_{Z}^{4}\left(m_{3}+m_{Z}\right)}{m_{3}^{3}\left(m_{3}^{2}-\xi m_{Z}^{2}\right)}-\frac{m_{3}}{m_{Z}}-1\right) \\
& +(e B)^{2} \frac{g^{2} T}{\pi}\left(\frac{1}{\left(m_{1}+m_{W}\right)^{4}}\left(-\frac{9 m_{1}}{16}-\frac{7 m_{1}^{3}}{32 m_{W}^{2}}-\frac{9 m_{1}^{2}}{16 m_{W}}-\frac{13 m_{W}}{48}-\frac{5 m_{W}^{2}}{96 m_{1}}\right)\right. \\
& +\frac{1}{\left(m_{1}+m_{W}\right)^{3}}\left(\frac{31}{192}+\frac{11 m_{1}^{2}}{64 m_{W}^{2}}+\frac{13 m_{1}}{64 m_{W}}+\frac{3 m_{W}}{64 m_{1}}\right)+\frac{1}{m_{W}\left(m_{1}+m_{W}\right)^{2}}\left(\frac{m_{1}}{24 m_{W}}+\frac{1}{48}\right) \\
& +\frac{m_{1}}{\left(m_{1}+\xi^{1 / 2} m_{W}\right)^{4}}\left(\frac{49 \xi}{96}+\frac{7 m_{1}^{2}}{32 m_{W}^{2}}+\frac{49 \xi^{1 / 2} m_{1}}{96 m_{W}}+\frac{7 \xi^{3 / 2} m_{W}}{32 m_{1}}\right) \\
& -\frac{1}{\left(m_{1}+\xi^{1 / 2} m_{W}\right)^{3}}\left(\frac{11 \xi}{64}+\frac{11 m_{1}^{2}}{64 m_{W}^{2}}+\frac{9 \xi^{1 / 2} m_{1}}{32 m_{W}}\right) \\
& \left.-\frac{1}{m_{W}\left(m_{1}+\xi^{1 / 2} m_{W}\right)^{2}}\left(\frac{m_{1}}{24 m_{W}}+\frac{\xi^{1 / 2}}{48}\right)\right)-\frac{7(e B)^{2} g^{2} \zeta(3)}{384 \pi^{4} T^{2}} \\
\Pi_{e}^{H}= & -\frac{\lambda^{2} m_{W}^{2}}{g^{2} \pi}\left[\frac{12}{\pi} \ln \left(\frac{m_{W}}{T}\right)+T\left(\frac{2}{m_{1}}+\frac{1}{m_{3}}+\frac{9}{m_{4}}\right)\right]+\frac{(e B)^{2} \lambda^{2} m_{W}^{2}}{4 g^{2} \pi m_{1}^{4}}\left[\frac{T}{m_{1}}+\frac{\zeta(5) m_{1}^{4}}{16 \pi^{5} T^{4}}\right] \\
\Pi_{f}^{H}= & \frac{m_{t}^{2} T^{2}}{2 v_{0}^{2}}\left[1+\frac{3 m_{t}^{2}}{\pi^{2} T^{2}} \ln \left(\frac{4 m_{W}}{T}\right)\right]-\frac{(e B)^{2} m_{t}^{2}}{2 \pi^{4} T^{2} v_{0}^{2}}\left[\frac{7 \zeta(3)}{9}-\frac{31 m_{t}^{2} \zeta(5)}{16 \pi^{2} T^{2}}\right] \\
\Pi_{g}^{H}= & \frac{g^{2} \xi^{2} m_{W}^{2}}{16 \pi}\left[\frac{1}{2 \pi}\left(3+2 \frac{g^{\prime 2}}{g^{2}}+\frac{g^{4}}{g^{4}}\right) \ln \left(\frac{m_{W}}{T}\right)+\frac{T}{m_{W}}+\left(1+2 \frac{g^{\prime 2}}{g^{2}}+\frac{g^{\prime 4}}{g^{4}}\right) \frac{T}{\left.2 m_{W}\right]}\right. \\
& -\frac{(e B)^{2} g^{2} \xi^{2} m_{W}^{2}}{128 \pi m_{W}^{4}}\left[\frac{T}{m_{W}}+\frac{\zeta(5) m_{W}^{4}}{16 \pi^{5} T^{4}}\right]
\end{aligned}
$$

\section{Neutral Goldstone boson $\left(G^{0}\right)$ self-energy in arbitrary gauge}

$$
\begin{aligned}
\Pi_{a}^{G^{0}}= & \frac{\lambda T^{2}}{2}\left[1-\frac{1}{2 \pi T}\left(2 m_{1}+3 m_{3}+m_{4}\right)-\frac{(e B)^{2}}{24 \pi T m_{1}^{3}}-\frac{(e B)^{2} \zeta(3)}{96 \pi^{4} T^{4}}\right] \\
\Pi_{b}^{G^{0}}= & \frac{T^{2}}{16}\left[(3+\xi) g^{2}+\left(1+\frac{\xi}{3}\right) g^{\prime 2}\right]-\frac{T}{16 \pi}\left[\left(6+2 \xi^{3 / 2}\right) g^{2} m_{W}+\left(g^{2}+g^{\prime 2}\right)\left(3+\xi^{3 / 2}\right) m_{Z}\right] \\
& +(e B)^{2} T \frac{g^{2}}{\pi m_{W}^{3}}\left(\frac{3}{64}+\frac{35}{192 \xi^{1 / 2}}+\frac{11 \zeta(3) m_{W}^{3}}{192 \pi^{3} T^{3}}\right) \\
\Pi_{d}^{G^{0}}= & -\left(3 g^{2}+g^{\prime 2}\right) \frac{\xi T^{2}}{48}-\frac{g^{2} T}{8 \pi}\left(\left(1+\frac{m_{1}}{m_{W}}\right) \frac{m_{1}^{3}}{m_{W}\left(m_{1}+m_{W}\right)}\right. \\
& \left.+\frac{1}{\left(m_{1}^{2}-\xi m_{W}^{2}\right)}\left(\xi^{5 / 2} m_{W}^{3}-\frac{m_{1}^{5}}{m_{W}^{2}}\right)+\left(1+\frac{g^{\prime 2}}{g^{2}}\right) \frac{m_{4}^{4}}{2 m_{Z}^{2}\left(m_{4}+m_{Z}\right)}\right) \\
& +(e B)^{2} \frac{g^{2} T}{\pi}\left(\frac{1}{\left(m_{1}+m_{W}\right)^{4}}\left(-\frac{9 m_{1}}{16}-\frac{7 m_{1}^{3}}{32 m_{W}^{2}}-\frac{9 m_{1}^{2}}{16 m_{W}}-\frac{13 m_{W}}{48}-\frac{5 m_{W}^{2}}{96}\right)\right.
\end{aligned}
$$




$$
\begin{aligned}
& +\frac{1}{\left(m_{1}+m_{W}\right)^{3}}\left(\frac{31}{192}+\frac{11 m_{1}^{2}}{64 m_{W}^{2}}+\frac{13 m_{1}}{64 m_{W}}+\frac{3 m_{W}}{64 m_{1}}\right)+\frac{1}{m_{W}\left(m_{1}+m_{W}\right)^{2}}\left(\frac{m_{1}}{24 m_{W}}+\frac{1}{48}\right) \\
& +\frac{m_{1}}{\left(m_{1}+\xi^{1 / 2} m_{W}\right)^{4}}\left(\frac{49 \xi}{96}+\frac{7 m_{1}^{2}}{32 m_{W}^{2}}+\frac{49 \xi^{1 / 2} m_{1}}{96 m_{W}}+\frac{7 \xi^{3 / 2} m_{W}}{32 m_{1}}\right) \\
& +\frac{1}{\left(m_{1}+\xi^{1 / 2} m_{W}\right)^{3}}\left(-\frac{11 \xi}{64}-\frac{11 m_{1}^{2}}{64 m_{W}^{2}}-\frac{9 \xi^{1 / 2} m_{1}}{32 m_{W}}\right) \\
& \left.+\frac{1}{m_{W}\left(m_{1}+\xi^{1 / 2} m_{W}\right)^{2}}\left(-\frac{m_{1}}{24 m_{W}}-\frac{\xi^{1 / 2}}{48}\right)\right) \\
\Pi_{e}^{G^{0}}= & -\frac{\lambda^{2} m_{W}^{2}}{g^{2}}\left[\frac{2}{\pi^{2}} \ln \left(\frac{m_{W}}{T}\right)+\frac{16 T}{4 \pi\left(m_{3}+m_{4}\right)}\right] \\
\Pi_{f}^{G^{0}}= & \frac{m_{t}^{2}}{2 v_{0}^{2}}\left[T^{2}-\frac{3 m_{t}^{2}}{\pi^{2}} \ln \left(\frac{4 m_{W}}{T}\right)-\frac{7(e B)^{2} \zeta(3)}{9 \pi^{4} T^{2}}+\frac{31(e B)^{2} m_{t}^{2} \zeta(5)}{16 \pi^{6} T^{4}}\right] \\
\Pi_{g}^{G^{0}}= & -\frac{g^{2} \xi^{2} m_{W}^{2}}{16 \pi}\left[\frac{1}{\pi} \ln \left(\frac{m_{W}}{T}\right)+\frac{T}{m_{W}}-\frac{(e B)^{2} T}{8 m_{W}^{5}}-\frac{(e B)^{2} \zeta(5)}{128 \pi^{5} T^{4}}\right]
\end{aligned}
$$

Charged Goldstone boson $\left(G^{c}\right)$ self-energy in arbitrary gauge

$$
\begin{aligned}
\Pi_{a}^{G^{c}}= & \frac{T^{2} \lambda}{2}\left[1-\frac{1}{2 \pi T}\left(2 m_{1}+m_{3}+m_{4}\right)-\frac{(e B)^{2}}{12 \pi T m_{1}^{3}}-\frac{(e B)^{2} \zeta(3)}{48 \pi^{4} T^{4}}\right] \\
\Pi_{b}^{G^{c}}= & T^{2} \frac{(\xi+3)\left(3 g^{4}+g^{\prime 4}+4 g^{2} g^{\prime 2}\right)}{48\left(g^{2}+g^{\prime 2}\right)} \\
& -T \frac{\left(\xi^{3 / 2}+3\right)}{4 \pi\left(g^{2}+g^{\prime 2}\right)}\left(\frac{g^{2}\left(g^{2}+g^{\prime 2}\right) m_{W}}{2}+\frac{\left(g^{2}-g^{\prime 2}\right)^{2} m_{Z}}{4}+g^{2} g^{\prime 2} m_{\gamma}\right) \\
& +(e B)^{2} T \frac{g^{2}}{\pi m_{W}^{3}}\left(\frac{3}{64}+\frac{35}{192 \xi^{1 / 2}}\right)+\frac{11(e B)^{2} g^{2} \zeta(3)}{192 \pi^{4} T^{2}} \\
\Pi_{c}^{G^{c}}= & -\frac{g^{\prime 2} m_{W}^{2}}{2 \pi^{2}} \ln \left(\frac{m_{W}}{T}\right) \\
& +\frac{T m_{W}^{2}}{4 \pi\left(g^{2}+g^{\prime 2}\right)}\left(-\frac{3 g^{\prime 4}}{\left(m_{W}+m_{Z}\right)}-\frac{g^{\prime 4}}{\left(\xi^{1 / 2} m_{W}+m_{Z}\right)}\left(\xi+\frac{\xi^{1 / 2} m_{Z}}{m_{W}}+\frac{m_{Z}^{2}}{m_{W}^{2}}\right)\right. \\
& -\frac{g^{\prime 4}}{\left(m_{W}+\xi^{1 / 2} m_{Z}\right)}\left(\xi+\frac{m_{W}^{2}}{m_{Z}^{2}}+\frac{\xi^{1 / 2} m_{W}}{m_{Z}}\right)+\frac{g^{\prime 4}}{\left(\xi m_{W}^{2}-m_{Z}^{2}\right)}\left(\frac{\xi^{5 / 2} m_{W}^{3}}{m_{Z}^{2}}-\frac{m_{Z}^{3}}{m_{W}^{2}}\right) \\
& +\frac{g^{\prime 4}}{\left(m_{W}^{2}-\xi m_{Z}^{2}\right)}\left(\frac{m_{W}^{3}}{m_{Z}^{2}}-\frac{\xi^{5 / 2} m_{Z}^{3}}{m_{W}^{2}}\right)-\frac{g^{\prime 4} \xi^{3 / 2}}{\left(m_{W}^{2}-m_{Z}^{2}\right)}\left(\frac{m_{W}^{3}}{m_{Z}^{2}}+\frac{m_{Z}^{3}}{m_{W}^{2}}\right) \\
& -\frac{3 g^{2} g^{\prime 2}}{\left(m_{W}+m_{\gamma}\right)}-\frac{g^{2} g^{\prime 2}}{\left(\xi^{1 / 2} m_{W}+m_{\gamma}\right)}\left(\xi+\frac{\xi^{1 / 2} m_{\gamma}}{\left.m_{W}+\frac{m_{\gamma}^{2}}{m_{W}^{2}}\right)}\right. \\
& -\frac{g^{2} g^{\prime 2}}{\left(m_{W}+\xi^{1 / 2} m_{\gamma}\right)}\left(\xi+\frac{m_{W}^{2}}{m_{\gamma}^{2}}+\frac{\xi^{1 / 2} m_{W}}{m_{\gamma}}\right)+\frac{g^{2} g^{\prime 2}}{\left(\xi m_{W}^{2}-m_{\gamma}^{2}\right)}\left(\frac{\xi^{5 / 2} m_{W}^{3}}{m_{\gamma}^{2}}-\frac{m_{\gamma}^{3}}{m_{W}^{2}}\right) \\
& \left.+\frac{g^{2} g^{\prime 2} m_{W}^{3}}{m_{\gamma}^{2}\left(m_{W}^{2}-\xi m_{\gamma}^{2}\right)}-\frac{g^{2} g^{\prime 2} \xi^{5 / 2} m_{\gamma}^{3}}{m_{W}^{2}\left(m_{W}^{2}-\xi m_{\gamma}^{2}\right)}-\frac{g^{2} g^{\prime 2} \xi^{3 / 2}}{\left(m_{W}^{2}-m_{\gamma}^{2}\right)}\left(\frac{m_{W}^{3}}{m_{\gamma}^{2}}-\frac{m_{\gamma}^{3}}{m_{W}^{2}}\right)\right) \\
&
\end{aligned}
$$




$$
\begin{aligned}
& +(e B)^{2} T \frac{m_{W}^{2}}{\pi\left(g^{2}+g^{\prime 2}\right)}\left(-\frac{g^{\prime 4}}{16\left(m_{W}+m_{Z}\right)^{4}}\left(\frac{13}{m_{W}}+\frac{2 m_{W}}{m_{Z}^{2}}+\frac{8}{m_{Z}}+\frac{12 m_{Z}}{m_{W}^{2}}+\frac{3 m_{Z}^{2}}{m_{W}^{3}}\right)\right. \\
& -\frac{g^{\prime 4}}{\left(m_{W}+m_{Z}\right)^{3}}\left(\frac{77}{96 m_{W}^{2}}-\frac{1}{24 m_{Z}^{2}}-\frac{1}{8 m_{W} m_{Z}}+\frac{5 m_{Z}}{32 m_{W}^{3}}\right) \\
& +\frac{g^{\prime 4}}{\left(m_{W}+m_{Z}\right)^{2}}\left(\frac{1}{4 m_{W}^{3}}+\frac{1}{24 m_{W} m_{Z}^{2}}+\frac{1}{12 m_{W}^{2} m_{Z}}\right) \\
& +\frac{5 g^{\prime 4}}{\left(\xi^{1 / 2} m_{W}+m_{Z}\right)^{4}}\left(\frac{\xi^{1 / 2}}{8 m_{W}}+\frac{\xi^{3 / 2} m_{W}}{48 m_{Z}^{2}}+\frac{\xi}{12 m_{Z}}+\frac{m_{Z}}{12 m_{W}^{2}}+\frac{m_{Z}^{2}}{48 \xi^{1 / 2} m_{W}^{3}}\right) \\
& -\frac{g^{\prime 4}}{\left(\xi^{1 / 2} m_{W}+m_{Z}\right)^{3}}\left(\frac{31}{96 m_{W}^{2}}+\frac{3 \xi}{32 m_{Z}^{2}}+\frac{9 \xi^{1 / 2}}{32 m_{W} m_{Z}}+\frac{7 m_{Z}}{32 \xi^{1 / 2} m_{W}^{3}}\right) \\
& -\frac{g^{\prime 4}}{\left(\xi^{1 / 2} m_{W}+m_{Z}\right)^{2}}\left(\frac{1}{4 \xi^{1 / 2} m_{W}^{3}}+\frac{\xi^{1 / 2}}{24 m_{W} m_{Z}^{2}}+\frac{1}{12 m_{W}^{2} m_{Z}}\right) \\
& +\frac{g^{\prime 4}}{\left(m_{W}+\xi^{1 / 2} m_{Z}\right)^{4}}\left(\frac{17 \xi}{24 m_{W}}+\frac{m_{W}}{8 m_{Z}^{2}}+\frac{\xi^{1 / 2}}{2 m_{Z}}+\frac{\xi^{3 / 2} m_{Z}}{3 m_{W}^{2}}\right) \\
& -\frac{g^{\prime 4}}{\left(m_{W}+\xi^{1 / 2} m_{Z}\right)^{3}}\left(\frac{\xi}{4 m_{W}^{2}}+\frac{1}{24 m_{Z}^{2}}+\frac{\xi^{1 / 2}}{8 m_{W} m_{Z}}\right)-\frac{g^{\prime 4}}{\left(m_{W}+\xi^{1 / 2} m_{Z}\right)^{2}}\left(\frac{1}{24 m_{W} m_{Z}^{2}}+\frac{\xi^{1 / 2}}{12 m_{W}^{2} m_{Z}}\right) \\
& -\frac{g^{\prime 4}}{\xi^{1 / 2}\left(m_{W}+m_{Z}\right)^{4}}\left(\frac{29}{48 m_{W}}+\frac{5 m_{W}}{48 m_{Z}^{2}}+\frac{5}{12 m_{Z}}+\frac{m_{Z}}{3 m_{W}^{2}}\right) \\
& +\frac{g^{\prime 4}}{\xi^{1 / 2}\left(m_{W}+m_{Z}\right)^{3}}\left(\frac{1}{4 m_{W}^{2}}+\frac{3}{32 m_{Z}^{2}}+\frac{9}{32 m_{W} m_{Z}}\right)+\frac{g^{\prime 4}}{12 \xi^{1 / 2}\left(m_{W}+m_{Z}\right)^{2}}\left(\frac{1}{2 m_{W} m_{Z}^{2}}+\frac{1}{m_{W}^{2} m_{Z}}\right) \\
& -\frac{g^{2} g^{\prime 2}}{\left(m_{W}+m_{\gamma}\right)^{4}}\left(\frac{13}{16 m_{W}}+\frac{m_{W}}{8 m_{\gamma}^{2}}+\frac{1}{2 m_{\gamma}}+\frac{3 m_{\gamma}}{4 m_{W}^{2}}+\frac{3 m_{\gamma}^{2}}{16 m_{W}^{3}}\right) \\
& -\frac{g^{2} g^{\prime} 2}{m_{W}^{2}\left(m_{W}+m_{\gamma}\right)^{3}}\left(\frac{77}{96 m_{W}^{2}}-\frac{1}{24 m_{\gamma}^{2}}-\frac{1}{8 m_{W} m_{\gamma}}+\frac{5 m_{\gamma}}{32 m_{W}^{3}}\right) \\
& +\frac{g^{2} g^{\prime 2}}{m_{W}\left(m_{W}+m_{\gamma}\right)^{2}}\left(\frac{1}{4 m_{W}^{2}}+\frac{1}{24 m_{\gamma}^{2}}+\frac{1}{12 m_{W} m_{\gamma}}\right) \\
& +\frac{5 g^{2} g^{\prime 2}}{\left(\xi^{1 / 2} m_{W}+m_{\gamma}\right)^{4}}\left(\frac{\xi^{3 / 2} m_{W}}{m_{\gamma}^{2}}+\frac{\xi}{12 m_{\gamma}}+\frac{m_{\gamma}}{12 m_{W}^{2}}+\frac{m_{\gamma}^{2}}{48 \xi^{1 / 2} m_{W}^{3}}+\frac{\xi^{1 / 2}}{8 m_{W}}\right) \\
& -\frac{g^{2} g^{\prime 2}}{\left(\xi^{1 / 2} m_{W}+m_{\gamma}\right)^{3}}\left(\frac{31}{96 m_{W}^{2}}+\frac{3 \xi}{32 m_{\gamma}^{2}}+\frac{9 \xi^{1 / 2}}{32 m_{W} m_{\gamma}}+\frac{7 m_{\gamma}}{32 \xi^{1 / 2} m_{W}^{3}}\right) \\
& -\frac{g^{2} g^{\prime 2}}{\left(\xi^{1 / 2} m_{W}+m_{\gamma}\right)^{2}}\left(\frac{1}{4 \xi^{1 / 2} m_{W}^{3}}+\frac{\xi^{1 / 2}}{24 m_{W} m_{\gamma}^{2}}+\frac{1}{12 m_{W}^{2} m_{\gamma}}\right) \\
& +\frac{g^{2} g^{\prime 2}}{\left(m_{W}+\xi^{1 / 2} m_{\gamma}\right)^{4}}\left(\frac{17 \xi}{24 m_{W}}+\frac{m_{W}}{8 m_{\gamma}^{2}}+\frac{\xi^{1 / 2}}{2 m_{\gamma}}+\frac{\xi^{3 / 2} m_{\gamma}}{3 m_{W}^{2}}\right) \\
& -\frac{g^{2} g^{\prime 2}}{\left(m_{W}+\xi^{1 / 2} m_{\gamma}\right)^{3}}\left(\frac{\xi}{4 m_{W}^{2}}+\frac{\xi^{1 / 2}}{8 m_{W} m_{\gamma}}+\frac{1}{24 m_{\gamma}^{2}}\right)-\frac{g^{2} g^{\prime 2}}{24 m_{W} m_{\gamma}\left(m_{W}+\xi^{1 / 2} m_{\gamma}\right)^{2}}\left(\frac{1}{m_{\gamma}}+\frac{2 \xi^{1 / 2}}{m_{W}}\right) \\
& -\frac{g^{2} g^{\prime 2}}{\xi^{1 / 2}\left(m_{W}+m_{\gamma}\right)^{4}}\left(\frac{29}{48 m_{W}}+\frac{5 m_{W}}{48 m_{\gamma}^{2}}+\frac{m_{\gamma}}{3 m_{W}^{2}}+\frac{5}{12 m_{\gamma}}\right) \\
& \left.+\frac{g^{2} g^{\prime 2}}{\xi^{1 / 2}\left(m_{W}+m_{\gamma}\right)^{3}}\left(\frac{1}{4 m_{W}^{2}}+\frac{3}{32 m_{\gamma}^{2}}+\frac{9}{32 m_{W} m_{\gamma}}\right)+\frac{g^{2} g^{\prime 2}}{\xi^{1 / 2}\left(m_{W}+m_{\gamma}\right)^{2}}\left(\frac{1}{24 m_{W} m_{\gamma}^{2}}+\frac{1}{12 m_{W}^{2} m_{\gamma}}\right)\right) \\
& -\frac{(e B)^{2}}{T^{4}} \frac{7 g^{2} m_{W}^{2} \zeta(5)}{512 \pi^{6}}
\end{aligned}
$$




$$
\begin{aligned}
& \Pi_{d}^{G^{c}}=-\frac{\xi\left(3 g^{4}+4 g^{2} g^{\prime 2}+g^{\prime 4}\right) T^{2}}{48\left(g^{2}+g^{\prime 2}\right)} \\
& +T\left(-\frac{g^{2} m_{3}^{3}}{16 \pi m_{W}^{2}}-\frac{g^{2} m_{4}^{3}}{16 \pi m_{W}^{2}}-\frac{\left(g^{2}-g^{\prime 2}\right)^{2} m_{1}^{3}}{16 \pi m_{Z}^{2}}-\frac{g^{2} g^{\prime 2} m_{1}^{3}}{4 \pi m_{\gamma}^{2}}\right. \\
& +\frac{g^{2}}{16 \pi\left(m_{3}^{2}-\xi m_{W}^{2}\right)}\left(\frac{m_{3}^{5}}{m_{W}^{2}}-\xi^{5 / 2} m_{W}^{3}\right)+\frac{g^{2}}{16 \pi\left(m_{4}^{2}-\xi m_{W}^{2}\right)}\left(\frac{m_{4}^{5}}{m_{W}^{2}}-\xi^{5 / 2} m_{W}^{3}\right) \\
& \left.+\frac{\left(g^{2}-g^{\prime 2}\right)^{2}}{16 \pi\left(m_{1}^{2}-\xi m_{Z}^{2}\right)}\left(\frac{m_{1}^{5}}{m_{Z}^{2}}-\xi^{5 / 2} m_{Z}^{3}\right)-\frac{g^{2} g^{\prime 2}}{4 \pi\left(m_{1}^{2}-\xi m_{\gamma}^{2}\right)}\left(\frac{m_{1}^{5}}{m_{\gamma}^{2}}-\xi^{5 / 2} m_{\gamma}^{3}\right)\right) \\
& +\frac{(e B)^{2} T}{\pi\left(g^{2}+g^{\prime 2}\right)}\left(-\frac{g^{2}}{\left(m_{3}+m_{W}\right)^{4}}\left(\frac{m_{3}}{8}+\frac{m_{3}^{3}}{12 m_{W}^{2}}+\frac{17 m_{3}^{2}}{96 m_{W}}+\frac{m_{W}}{32}\right)\right. \\
& +\frac{g^{2}}{\left(m_{3}+m_{W}\right)^{3}}\left(\frac{1}{96}+\frac{m_{3}^{2}}{16 m_{W}^{2}}+\frac{m_{3}}{32 m_{W}}\right)+\frac{g^{2}}{\pi\left(m_{4}+m_{W}\right)^{3}}\left(\frac{1}{96}+\frac{m_{4}^{2}}{16 m_{W}^{2}}+\frac{m_{4}}{32 m_{W}}\right) \\
& +\frac{g^{2}}{\left(m_{3}+m_{W}\right)^{2}}\left(\frac{1}{96}+\frac{m_{3}}{48 m_{W}}\right)+\frac{g^{2}}{m_{W}\left(m_{4}+m_{W}\right)^{2}}\left(\frac{1}{96}+\frac{m_{4}}{48 m_{W}}\right) \\
& -\frac{g^{2}}{\left(m_{4}+m_{W}\right)^{4}}\left(\frac{m_{4}}{8}+\frac{m_{4}^{3}}{12 m_{W}^{2}}+\frac{17 m_{4}^{2}}{96 m_{W}}+\frac{m_{W}}{32}\right) \\
& +\frac{g^{2}}{\left(m_{3}+\xi^{1 / 2} m_{W}\right)^{4}}\left(\frac{5 \xi m_{3}}{48}+\frac{m_{3}^{3}}{12 m_{W}^{2}}+\frac{29 \xi^{1 / 2} m_{3}^{2}}{192 m_{W}}+\frac{5 \xi^{3 / 2} m_{W}}{192}\right) \\
& -\frac{g^{2}}{\left(m_{3}+\xi^{1 / 2} m_{W}\right)^{3}}\left(\frac{3 \xi}{128}+\frac{m_{3}^{2}}{16 m_{W}^{2}}+\frac{9 \xi^{1 / 2} m_{3}}{128 m_{W}}\right) \\
& -\frac{g^{2}}{m_{W}\left(m_{3}+\xi^{1 / 2} m_{W}\right)^{2}}\left(\frac{m_{3}}{48 m_{W}}+\frac{\xi^{1 / 2}}{96}\right) \\
& +\frac{g^{2}}{\left(m_{4}+\xi^{1 / 2} m_{W}\right)^{4}}\left(\frac{5 \xi m_{4}}{48}+\frac{m_{4}^{3}}{12 m_{W}^{2}}+\frac{29 \xi^{1 / 2} m_{4}^{2}}{192 m_{W}}+\frac{5 \xi^{3 / 2} m_{W}}{192}\right) \\
& -\frac{g^{2}}{\left(m_{4}+\xi^{1 / 2} m_{W}\right)^{3}}\left(\frac{3 \xi}{128}+\frac{m_{4}^{2}}{16 m_{W}^{2}}+\frac{9 \xi^{1 / 2} m_{4}}{128 m_{W}}\right) \\
& -\frac{g^{2}}{m_{W}\left(m_{4}+\xi^{1 / 2} m_{W}\right)^{2}}\left(\frac{m_{4}}{48 m_{W}}+\frac{\xi^{1 / 2}}{96}\right) \\
& -\frac{5\left(g^{2}-g^{\prime 2}\right)^{2} m_{1}}{192\left(m_{1}+m_{Z}\right)^{4}}\left(6+\frac{m_{1}^{2}}{m_{Z}^{2}}+4 \frac{m_{1}}{m_{Z}}\right)+\frac{5\left(g^{2}-g^{\prime 2}\right)^{2} m_{Z}}{192\left(m_{1}+m_{Z}\right)^{4}}\left(4+\frac{m_{Z}}{m_{1}}\right) \\
& +\frac{9\left(g^{2}-g^{\prime 2}\right)^{2}}{128\left(m_{1}+m_{Z}\right)^{3}}\left(1+\frac{m_{1}^{2}}{3 m_{Z}^{2}}+\frac{m_{1}}{m_{Z}}+\frac{m_{Z}}{3 m_{1}}\right) \\
& +\frac{\left(g^{2}-g^{\prime 2}\right)^{2}}{\left(m_{1}+\xi^{1 / 2} m_{Z}\right)^{4}}\left(\frac{29 \xi m_{1}}{192}+\frac{5 m_{1}^{3}}{192 m_{Z}^{2}}+\frac{5 \xi^{1 / 2} m_{1}^{2}}{48 m_{Z}}+\frac{\xi^{3 / 2} m_{Z}}{12}\right) \\
& +\frac{\left(g^{2}-g^{\prime 2}\right)^{2}}{\left(m_{1}+\xi^{1 / 2} m_{Z}\right)^{3}}\left(-\frac{\xi}{16}-\frac{3 m_{1}^{2}}{128 m_{Z}^{2}}-\frac{9 \xi^{1 / 2} m_{1}}{128 m_{Z}}\right) \\
& -\frac{5 g^{2} g^{\prime 2}}{\left(m_{1}+m_{\gamma}\right)^{4}}\left(\frac{m_{1}}{8}+\frac{m_{1}^{3}}{48 m_{\gamma}^{2}}+\frac{m_{1}^{2}}{12 m_{\gamma}}+\frac{m_{\gamma}}{12}+\frac{m_{\gamma}^{2}}{48 m_{1}}\right) \\
& +\frac{g^{2} g^{\prime 2}}{\left(m_{1}+m_{\gamma}\right)^{3}}\left(+\frac{9}{32}+\frac{3 m_{1}^{2}}{32 m_{\gamma}^{2}}+\frac{9 m_{1}}{32 m_{\gamma}}+\frac{3 m_{\gamma}}{32 m_{1}}\right) \\
& +\frac{g^{2} g^{\prime 2}}{\left(m_{1}+\xi^{1 / 2} m_{\gamma}\right)^{4}}\left(+\frac{29 \xi m_{1}}{48}+\frac{5 m_{1}^{3}}{48 m_{\gamma}^{2}}+\frac{5 \xi^{1 / 2} m_{1}^{2}}{12 m_{\gamma}}+\frac{\xi^{3 / 2} m_{\gamma}}{3}\right) \\
& \left.-\frac{g^{2} g^{\prime 2}}{\left(m_{1}+\xi^{1 / 2} m_{\gamma}\right)^{3}}\left(-\frac{\xi}{4}-\frac{3 m_{1}^{2}}{32 m_{\gamma}^{2}}-\frac{9 \xi^{1 / 2} m_{1}}{32 m_{\gamma}}\right)\right) \\
& +\frac{(e B)^{2}}{T^{2}} \frac{\left(g^{\prime 2}-29 g^{2}\right) \zeta(3)}{1536 \pi^{4}}
\end{aligned}
$$




$$
\begin{aligned}
\Pi_{e}^{G^{c}}= & \frac{\lambda^{2} m_{W}^{2}}{g^{2}}\left[-\frac{2}{\pi^{2}} \ln \left(\frac{m_{W}}{T}\right)-\frac{4 T}{\pi\left(m_{1}+m_{4}\right)}\right. \\
& \left.+\frac{(e B)^{2} T}{\pi m_{1}\left(m_{1}+m_{4}\right)^{4}}\left(-\frac{1}{3}-\frac{4 m_{4}}{3 m_{1}}-\frac{m_{4}^{2}}{3 m_{1}^{2}}+\frac{3\left(m_{1}+m_{4}\right)}{2 m_{1}}+\frac{m_{4}\left(m_{1}+m_{4}\right)}{2 m_{1}^{2}}\right)+\frac{(e B)^{2} \zeta(5)}{128 \pi^{6} T^{4}}\right] \\
\Pi_{f}^{G^{c}}= & \frac{1}{v_{0}^{2}}\left[\frac{T^{2}}{2}\left(m_{b}^{2}+m_{t}^{2}\right)+\frac{(e B)}{T} \frac{3\left(m_{b}^{2}-m_{t}^{2}\right)}{16 \pi}-\frac{3 m_{b}^{2} m_{t}^{2}}{\pi^{2}} \ln \left(\frac{4 m_{W}}{T}\right)\right. \\
& \left.-\frac{(e B)^{2}}{T^{2}} \frac{133\left(m_{b}^{2}+m_{t}^{2}\right) \zeta(3)}{288 \pi^{4}}+\frac{(e B)^{2}}{T^{4}} \frac{31 \zeta(5)}{256 \pi^{6}}\left(m_{b}^{4}+2 m_{b}^{2} m_{t}^{2}+4 m_{t}^{4}\right)\right] \\
\Pi_{g}^{G^{c}}= & -\left(g^{2}-g^{\prime 2}\right)\left(\frac{\xi^{2} m_{W}^{2}}{16 \pi^{2}} \ln \left(\frac{m_{W}}{T}\right)-\frac{\xi^{2} T}{8 \pi\left(m_{W}+m_{Z}\right)}\right. \\
& +\frac{(e B)^{2} \xi^{2} T m_{W}^{2}}{\pi m_{W}\left(m_{W}+m_{Z}\right)^{4}}\left(\frac{1}{96}+\frac{m_{Z}}{24 m_{W}}+\frac{m_{Z}^{2}}{96 m_{W}^{2}}-\frac{3\left(m_{W}+m_{Z}\right)}{64 m_{W}}-\frac{m_{Z}\left(m_{W}+m_{Z}\right)}{64 m_{W}^{2}}\right) \\
& \left.-\frac{(e B)^{2} \xi^{2} \zeta(5)}{4096 \pi^{6} T^{4}}\right)
\end{aligned}
$$

\section{APPENDIX B: GAUGE BOSON SELF-ENERGIES IN ARBITRARY GAUGE}

In this appendix, we list the results for the self-energy diagrams depicted in Fig. 2

\section{Photon $(\gamma)$ self-energy in arbitrary gauge}

$$
\begin{aligned}
\Pi_{a}^{\gamma}= & \frac{g^{2} g^{\prime 2}}{\left(g^{2}+g^{\prime 2}\right)}\left[T^{2}\left(\frac{\xi+1}{4}-\left(\xi^{3 / 2}+2\right) \frac{m_{W}}{2 \pi T}\right)+\frac{(e B)^{2}}{16 \pi m_{W}^{2}}\left(\left(\frac{1}{\xi^{1 / 2}}-2\right) \frac{T}{3 m_{W}}+\frac{7 \zeta(3) m_{W}^{2}}{4 \pi^{3} T^{2}}\right)\right] \\
\Pi_{b}^{\gamma}= & -\frac{g^{2} g^{\prime 2} m_{W}^{2}}{\left(g^{2}+g^{\prime 2}\right)}\left[\frac{1}{4 \pi^{2}} \ln \left(\frac{m_{W}}{T}\right)+\frac{2 T}{4 \pi\left(m_{1}+m_{W}\right)}\right. \\
& -\frac{(e B)^{2} T}{4 \pi\left(m_{1}+m_{W}\right)^{2}}\left(\frac{1}{m_{W}^{3}}+\frac{1}{4\left(m_{1}+m_{W}\right)}\left(\frac{3}{m_{1}^{2}}-\frac{7 m_{1}}{m_{W}^{3}}-\frac{21}{m_{W}^{2}}+\frac{m_{W}}{m_{1}^{3}}\right)-\frac{\left(m_{1}+m_{W}\right)^{2}}{\xi^{1 / 2} m_{W}^{3}\left(m_{1}+\xi^{1 / 2} m_{W}\right)^{2}}\right. \\
& \left.\left.-\frac{1}{6\left(m_{1}+m_{W}\right)^{2}}\left(\frac{1}{m_{1}}+\frac{1}{m_{W}}+\frac{m_{1}^{2}}{m_{W}^{3}}+\frac{m_{W}^{2}}{m_{1}^{3}}+\frac{4 m_{1}}{m_{W}^{2}}+\frac{4 m_{W}}{m_{1}^{2}}\right)\right)+\frac{7(e B)^{2} \zeta(5)}{512 \pi^{6} T^{4}}\right] \\
\Pi_{c}^{\gamma}= & \frac{g^{2} g^{\prime 2} T^{2}}{2\left(g^{2}+g^{\prime 2}\right)}\left[\frac{1}{3}-\frac{m_{1}}{\pi T}\right]-\frac{g^{2} g^{\prime 2}(e B)^{2}}{48\left(g^{2}+g^{\prime 2}\right) \pi m_{1}^{2}}\left[\frac{T}{m_{1}}+\frac{\zeta(3) m_{1}^{2}}{4 \pi^{3} T^{2}}\right] \\
\Pi_{d}^{\gamma}= & \frac{g^{2} g^{\prime 2}}{\left(g^{2}+g^{\prime 2}\right)}\left[\frac{(2-\xi) T^{2}}{4}+\frac{\xi T m_{W}}{2 \pi\left(1+\xi^{1 / 2}\right)}\left(1+\xi^{1 / 2}+\xi\right)\right. \\
& +\frac{(e B)^{2} T}{2 \pi m_{W}^{3}}\left(\frac{1}{4\left(1+\xi^{1 / 2}\right)^{2}}\left(-\frac{37}{2}\left(1+\xi^{1 / 2}\right)^{2}+\frac{7}{\left(1+\xi^{1 / 2}\right)^{2}}+\frac{13}{2\left(1+\xi^{1 / 2}\right)}+\frac{14}{3}\right)\right. \\
& +\frac{\xi^{1 / 2}}{\left(1+\xi^{1 / 2}\right)^{2}}\left(-\frac{15}{8}\left(1+\xi^{1 / 2}\right)^{2}+\frac{49}{12\left(1+\xi^{1 / 2}\right)^{2}}+\frac{27}{4\left(1+\xi^{1 / 2}\right)}+\frac{13}{3}\right) \\
& \left.+\frac{\xi}{\left(1+\xi^{1 / 2}\right)^{2}}\left(\frac{49}{12\left(1+\xi^{1 / 2}\right)^{2}}+\frac{53}{8\left(1+\xi^{1 / 2}\right)}+5\right)+\frac{\xi^{3 / 2}}{2\left(1+\xi^{1 / 2}\right)^{2}}\left(\frac{7}{2\left(1+\xi^{1 / 2}\right)^{2}}+5\right)\right) \\
& \left.-\frac{157(e B)^{2} \zeta(3)}{384 \pi^{4} T^{2}}\right]
\end{aligned}
$$




$$
\begin{aligned}
& \Pi_{e}^{\gamma}=\frac{g^{2} g^{\prime 2}}{\left(g^{2}+g^{\prime 2}\right)}\left(\frac{T^{2}}{6}+\frac{(e B)^{2} \zeta(3)}{64 \pi^{4} T^{2}}\right) \\
& \Pi_{f}^{\gamma}=\frac{g^{2} g^{\prime 2}}{\left(g^{2}+g^{\prime 2}\right)}\left(\frac{4 T^{2}}{9}-\frac{2 m_{t}^{2}}{3 \pi^{2}} \ln \left(\frac{4 m_{W}}{T}\right)+\frac{7(e B)^{2} \zeta(3)}{81 \pi^{4} T^{2}}-\frac{31(e B)^{2} m_{t}^{2} \zeta(5)}{108 \pi^{6} T^{4}}\right) \\
& \Pi_{g}^{\gamma}=-\frac{g^{2} g^{\prime 2}}{\left(g^{2}+g^{\prime 2}\right)}\left(\frac{T^{2}}{12}+\frac{(e B)^{2} \zeta(3)}{128 \pi^{4} T^{2}}\right)
\end{aligned}
$$

\section{Neutral gauge boson $(Z)$ self-energy in arbitrary gauge}

$$
\begin{aligned}
& \Pi_{a}^{Z}=\left(\frac{g^{4}}{g^{2}+g^{\prime 2}}\right)\left\{\frac{(1+\xi)}{4} T^{2}-\left(\frac{4+2 \xi^{3 / 2}}{4 \pi}\right) T m_{W}+\frac{(e B)^{2}}{16 \pi m_{W}^{2}}\left[\left(\frac{23-2 \xi^{1 / 2}}{3 \xi^{1 / 2}}\right) \frac{T}{m_{W}}+\frac{7 \zeta(3)}{4 \pi^{3}} \frac{m_{W}^{2}}{T^{2}}\right]\right\} \\
& \Pi_{b}^{Z}=-\frac{\left(g^{6}+3 g^{4} g^{\prime 2}+5 g^{2} g^{\prime 4}+g^{\prime 6}\right) m_{W}^{2}}{8 g^{2}\left(g^{2}+g^{\prime 2}\right) \pi^{2}} \ln \left(\frac{m_{W}}{T}\right)-\frac{T m_{W}^{2}}{4 \pi\left(m_{4}+m_{Z}\right)}\left(\frac{2 g^{\prime 4}}{\left(g^{2}+g^{\prime 2}\right)} \frac{\left(m_{4}+m_{Z}\right)}{\left(m_{1}+m_{W}\right)}\right. \\
& \left.+\frac{\left(g^{2}+g^{\prime 2}\right)^{2}}{g^{2}}\right)+\frac{g^{\prime 4} m_{W}^{2}(e B)^{2}}{\left(g^{2}+g^{\prime 2}\right)\left(m_{1}+m_{W}\right)^{4}}\left[\frac { T } { 4 8 \pi m _ { 1 } } \left(7-\frac{m_{1}^{3}}{m_{W}^{3}}-68 \frac{m_{1}^{2}}{m_{W}^{2}}-53 \frac{m_{1}}{m_{W}}+4 \frac{m_{W}}{m_{1}}+\frac{m_{W}^{2}}{m_{1}^{2}}\right.\right. \\
& \left.\left.-\frac{12}{\xi^{1 / 2}} \frac{m_{1}\left(m_{1}+m_{W}\right)^{4}}{m_{W}^{3}\left(m_{1}+\xi^{1 / 2} m_{W}\right)^{2}}\right)-\frac{7 \zeta(5)}{512 \pi^{6}} \frac{\left(m_{1}+m_{W}\right)^{4}}{T^{4}}\right] \\
& \Pi_{c}^{Z}=\frac{g^{4}+g^{\prime 4}}{g^{2}+g^{\prime 2}} \frac{T^{2}}{12}-\left[\frac{2\left(g^{2}-g^{\prime 2}\right)^{2} m_{1}+\left(g^{2}+g^{\prime 2}\right)^{2}\left(m_{3}+m_{4}\right)}{g^{2}+g^{\prime 2}}\right] \frac{T}{16 \pi}-\frac{\left(g^{2}-g^{\prime 2}\right)^{2}}{g^{2}+g^{\prime 2}} \frac{(e B)^{2}}{192 \pi m_{1}^{2}}\left[\frac{T}{m_{1}}+\frac{\zeta(3)}{4 \pi^{3}} \frac{m_{1}^{2}}{T^{2}}\right] \\
& \Pi_{d}^{Z}=\frac{g^{4}}{g^{2}+g^{\prime 2}}\left\{\left(1-\frac{\xi}{2}\right) \frac{T^{2}}{2}+\frac{\xi\left(1+\xi^{1 / 2}+\xi\right)}{2 \pi\left(1+\xi^{1 / 2}\right)} T m_{W}+\frac{1}{16 \pi}\left[\frac{14}{\left(1+\xi^{1 / 2}\right)^{4}}\left(1+\frac{7}{3} \xi^{1 / 2}+\frac{7}{3} \xi+\xi^{3 / 2}\right)\right.\right. \\
& +\frac{1}{\left(1+\xi^{1 / 2}\right)^{3}}\left(13+54 \xi^{1 / 2}+53 \xi\right)+\frac{8}{\left(1+\xi^{1 / 2}\right)^{2}}\left(\frac{7}{6}+\frac{13 \xi^{1 / 2}}{3}+5 \xi+\frac{5 \xi^{3 / 2}}{2}\right) \\
& \left.\left.-\left(37+15 \xi^{1 / 2}\right)-\frac{157 \zeta(3)}{24 \pi^{3}} \frac{m_{W}^{3}}{T^{3}}\right] \frac{(e B)^{2} T}{m_{W}^{3}}\right\} \\
& \Pi_{e}^{Z}=\left(\frac{g^{4}+g^{4}}{g^{2}+g^{\prime 2}}\right) \frac{T^{2}}{12}+\frac{\left(g^{2}-g^{\prime 2}\right)^{2}}{g^{2}+g^{\prime 2}} \frac{\zeta(3)}{256 \pi^{4}} \frac{(e B)^{2}}{T^{2}} \\
& \Pi_{f}^{Z}=\frac{g^{\prime 2}\left(3 g^{2}-g^{\prime 2}\right)}{6\left(g^{2}+g^{\prime 2}\right) \pi^{2}} m_{t}^{2} \ln \left(\frac{4 m_{W}}{T}\right) \\
& +\left(-\frac{g^{\prime 2}}{3}+\frac{4 g^{\prime 4}}{9\left(g^{2}+g^{\prime 2}\right)}+\frac{1}{8}\left(g^{2}+g^{\prime 2}\right)\right)\left\{T^{2}-\frac{(e B)^{2}}{T^{2}}\left[\frac{31 \zeta(5)}{48 \pi^{6}} \frac{m_{t}^{2}}{T^{2}}-\frac{7 \zeta(3)}{36 \pi^{4}}\right]\right\} \\
& \Pi_{g}^{Z}=-\frac{g^{4}}{\left(g^{2}+g^{\prime 2}\right)}\left[\frac{T^{2}}{12}+\frac{\zeta(3)}{128 \pi^{4}} \frac{(e B)^{2}}{T^{2}}\right]
\end{aligned}
$$




\section{Charged gauge boson $(W)$ self-energy in arbitrary gauge}

$$
\begin{aligned}
& \Pi_{a}^{W}=2 g^{2} T^{2}(\xi+1)-\frac{\left(2+\xi^{3 / 2}\right) g^{2} T}{4 \pi\left(g^{2}+g^{\prime 2}\right)}\left(\left(g^{2}+g^{\prime 2}\right) m_{W}+g^{2} m_{Z}+g^{\prime 2} m_{\gamma}\right) \\
& -\frac{g^{2}(e B)^{2}}{128 \pi m_{W}^{2}}\left(\frac{4 T}{3 m_{W}}\left(2-\frac{23}{\xi^{1 / 2}}\right)+\frac{7 \zeta(3) m_{W}^{2}}{\pi^{3} T^{2}}\right) \\
& \Pi_{b}^{W}=-\frac{\left(g^{2}+g^{\prime 2}\right) m_{W}^{2}}{8 \pi^{2}} \ln \left(\frac{m_{W}}{T}\right)-\frac{T m_{W}^{2}}{4 \pi\left(g^{2}+g^{\prime 2}\right)}\left(\frac{g^{2}\left(g^{2}+g^{\prime 2}\right)}{m_{4}+m_{W}}+\frac{g^{\prime 4}}{m_{1}+m_{Z}}+\frac{g^{2} g^{\prime 2}}{m_{1}+m_{\gamma}}\right) \\
& +(e B)^{2} T\left(-\frac{g^{2} m_{W}}{48 \pi\left(m_{4}+m_{W}\right)^{4}}\left(\frac{m_{4}^{2}}{m_{W}^{2}}+\frac{4 m_{4}}{m_{W}}+1\right)\right. \\
& -\frac{7 g^{2}}{32 \pi\left(m_{4}+m_{W}\right)^{3}}\left(\frac{m_{4}}{m_{W}}+3\right)+\frac{g^{2}}{8 \pi m_{W}}\left(\frac{1}{\left(m_{4}+m_{W}\right)^{2}}-\frac{1}{\xi^{1 / 2}\left(m_{4}+\xi^{1 / 2} m_{W}\right)^{2}}\right) \\
& -\frac{g^{\prime 4} m_{W}^{2}}{48 \pi m_{1}\left(g^{2}+g^{\prime 2}\right)\left(m_{1}+m_{Z}\right)^{4}}\left(1+\frac{4 m_{Z}}{m_{1}}+\frac{m_{Z}^{2}}{m_{1}^{2}}\right)+\frac{g^{\prime 4} m_{W}^{2}}{32\left(g^{2}+g^{\prime 2}\right) \pi m_{1}^{2}\left(m_{1}+m_{Z}\right)^{3}}\left(3+\frac{m_{Z}}{m_{1}}\right) \\
& \left.-\frac{g^{2} g^{\prime 2} m_{W}^{2}}{48\left(g^{2}+g^{\prime 2}\right) \pi m_{1}\left(m_{1}+m_{\gamma}\right)^{4}}\left(1+\frac{4 m_{\gamma}}{m_{1}}+\frac{m_{\gamma}^{2}}{m_{1}^{2}}\right)+\frac{g^{2} g^{\prime 2} m_{W}^{2}}{32\left(g^{2}+g^{\prime 2}\right) \pi m_{1}^{2}\left(m_{1}+m_{\gamma}\right)^{3}}\left(3+\frac{m_{\gamma}}{m_{1}}\right)\right) \\
& +\frac{(e B)^{2} m_{W}^{2} \zeta(5)}{2048 \pi^{6} T^{4}}\left(g^{\prime 4}-15 g^{4}-14 g^{2} g^{\prime 2}\right) \\
& \Pi_{c}^{W}=\frac{g^{2} T^{2}}{12}-\frac{g^{2} T}{16 \pi}\left(2 m_{1}+m_{3}+m_{4}\right)-\frac{g^{2}(e B)^{2}}{192 \pi m_{1}^{2}}\left(\frac{T}{m_{1}}+\frac{\zeta(3) m_{1}^{2}}{4 \pi^{3} T^{2}}\right) \\
& \Pi_{d}^{W}=\frac{T^{2} g^{2}}{4}(2-\xi)+\frac{T}{4 \pi\left(g^{2}+g^{\prime 2}\right)}\left(-\frac{g^{4}}{\left(m_{W}+m_{Z}\right)}\left(\frac{m_{W}^{4}}{m_{Z}^{2}}+\frac{m_{W}^{3}}{m_{Z}}+\frac{m_{Z}^{3}}{m_{W}}+\frac{m_{Z}^{4}}{m_{W}^{2}}\right)\right. \\
& +\frac{g^{4}}{\left(\xi m_{W}^{2}-m_{Z}^{2}\right)}\left(\xi^{5 / 2}\left(m_{W}^{3}-m_{Z}^{3}\right)+\frac{m_{W}^{5}}{m_{Z}^{2}}-\frac{m_{Z}^{5}}{m_{W}^{2}}\right)-\frac{g^{2} g^{\prime 2}}{\left(m_{W}+m_{\gamma}\right)}\left(\frac{m_{W}^{4}}{m_{\gamma}^{2}}+\frac{m_{W}^{3}}{m_{\gamma}}+\frac{m_{\gamma}^{3}}{m_{W}}+\frac{m_{\gamma}^{4}}{m_{W}^{2}}\right) \\
& \left.+\frac{g^{2} g^{\prime 2}}{\left(\xi m_{W}^{2}-m_{\gamma}^{2}\right)}\left(\xi^{5 / 2} m_{W}^{3}-\frac{m_{\gamma}^{5}}{m_{W}^{2}}\right)+\frac{g^{2} g^{\prime 2}}{\left(\xi m_{\gamma}^{2}-m_{W}^{2}\right)}\left(\xi^{5 / 2} m_{\gamma}^{3}-\frac{m_{W}^{5}}{m_{\gamma}^{2}}\right)\right) \\
& +\frac{(e B)^{2} T}{\pi\left(g^{2}+g^{\prime 2}\right)}\left(-\frac{g^{4}}{\left(m_{W}+m_{Z}\right)^{4}}\left(\frac{3 m_{W}}{4}+\frac{5 m_{W}^{3}}{48 m_{Z}^{2}}+\frac{5 m_{W}^{2}}{12 m_{Z}}+\frac{11 m_{Z}}{12}+\frac{13 m_{Z}^{2}}{16 m_{W}}+\frac{m_{Z}^{3}}{3 m_{W}^{2}}\right)\right. \\
& -\frac{g^{4}}{\left(m_{W}+m_{Z}\right)^{3}}\left(\frac{185}{96}+\frac{21 m_{W}^{2}}{32 m_{Z}^{2}}+\frac{63 m_{W}}{32 m_{Z}}+\frac{17 m_{Z}}{32 m_{W}}-\frac{m_{Z}^{2}}{4 m_{W}^{2}}\right) \\
& -\frac{g^{4}}{\left(m_{W}+m_{Z}\right)^{2}}\left(\frac{1}{3 m_{W}}+\frac{3 m_{W}}{8 m_{Z}^{2}}+\frac{3}{4 m_{Z}}-\frac{m_{Z}}{12 m_{W}^{2}}\right)+\frac{g^{4}}{\left(m_{W}+\xi^{1 / 2} m_{Z}\right)^{3}}\left(\frac{7 \xi}{4}+\frac{21 m_{W}^{2}}{32 m_{Z}^{2}}+\frac{63 \xi^{1 / 2} m_{W}}{32 m_{Z}}\right) \\
& +\frac{g^{4}}{\left(\xi^{1 / 2} m_{W}+m_{Z}\right)^{4}}\left(\frac{5 \xi^{3 / 2} m_{W}}{48}+\frac{5 \xi m_{Z}}{12}+\frac{29 \xi^{1 / 2} m_{Z}^{2}}{48 m_{W}}+\frac{m_{Z}^{3}}{3 m_{W}^{2}}\right) \\
& -\frac{g^{4}}{\left(\xi^{1 / 2} m_{W}+m_{Z}\right)^{3}}\left(\frac{3 \xi}{32}+\frac{9 \xi^{1 / 2} m_{Z}}{32 m_{W}}+\frac{m_{Z}^{2}}{4 m_{W}^{2}}\right)+\frac{g^{4}}{\left(\xi^{1 / 2} m_{W}+m_{Z}\right)^{2}}\left(\frac{\xi^{1 / 2}}{3 m_{W}}+\frac{3 \xi^{3 / 2} m_{W}}{8 m_{Z}^{2}}+\frac{3 \xi}{4 m_{Z}}-\frac{m_{Z}}{12 m_{W}^{2}}\right) \\
& +\frac{g^{4}}{\left(m_{W}+\xi^{1 / 2} m_{Z}\right)^{4}}\left(\frac{29 \xi m_{W}}{48}+\frac{5 m_{W}^{3}}{48 m_{Z}^{2}}+\frac{5 \xi^{1 / 2} m_{W}^{2}}{12 m_{Z}}+\frac{\xi^{3 / 2} m_{Z}}{3}\right) \\
& +\frac{g^{4}}{\left(m_{W}+\xi^{1 / 2} m_{Z}\right)^{2}}\left(\frac{\xi}{2 m_{W}}+\frac{3 m_{W}}{8 m_{Z}^{2}}+\frac{3 \xi^{1 / 2}}{4 m_{Z}}+\frac{\xi^{3 / 2} m_{Z}}{4 m_{W}^{2}}\right) \\
& -\frac{g^{4}}{\left(\xi^{1 / 2} m_{W}+\xi^{1 / 2} m_{Z}\right)^{2}}\left(\frac{\xi^{3 / 2}}{2 m_{W}}+\frac{3 \xi^{3 / 2} m_{W}}{8 m_{Z}^{2}}+\frac{3 \xi^{3 / 2}}{4 m_{Z}}+\frac{\xi^{3 / 2} m_{Z}}{4 m_{W}^{2}}\right) \\
& -\frac{g^{2} g^{\prime 2}}{\left(m_{W}+m_{\gamma}\right)^{4}}\left(\frac{3 m_{W}}{4}+\frac{5 m_{W}^{3}}{48 m_{\gamma}^{2}}+\frac{5 m_{W}^{2}}{12 m_{\gamma}}+\frac{11 m_{\gamma}}{12}+\frac{13 m_{\gamma}^{2}}{16 m_{W}}+\frac{m_{\gamma}^{3}}{3 m_{W}^{2}}\right) \\
& -\frac{g^{2} g^{\prime 2}}{\left(m_{W}+m_{\gamma}\right)^{3}}\left(\frac{185}{96}+\frac{21 m_{W}^{2}}{32 m_{\gamma}^{2}}+\frac{63 m_{W}}{32 m_{\gamma}}+\frac{17 m_{\gamma}}{32 m_{W}}-\frac{m_{\gamma}^{2}}{4 m_{W}^{2}}\right)
\end{aligned}
$$




$$
\begin{aligned}
& -\frac{g^{2} g^{\prime 2}}{\left(m_{W}+m_{\gamma}\right)^{2}}\left(\frac{1}{3 m_{W}}+\frac{3 m_{W}}{8 m_{\gamma}^{2}}+\frac{3}{4 m_{\gamma}}-\frac{m_{\gamma}}{12 m_{W}^{2}}\right) \\
& +\frac{g^{2} g^{\prime 2}}{\left(\xi^{1 / 2} m_{W}+m_{\gamma}\right)^{4}}\left(\frac{5 \xi^{3 / 2} m_{W}}{48}+\frac{5 \xi m_{\gamma}}{12}+\frac{29 \xi^{1 / 2} m_{\gamma}^{2}}{48 m_{W}}+\frac{m_{\gamma}^{3}}{3 m_{W}^{2}}\right) \\
& +\frac{g^{2} g^{\prime 2}}{\left(\xi^{1 / 2} m_{W}+m_{\gamma}\right)^{2}}\left(\frac{\xi^{1 / 2}}{3 m_{W}}+\frac{3 \xi^{3 / 2} m_{W}}{8 m_{\gamma}^{2}}+\frac{3 \xi}{4 m_{\gamma}}-\frac{m_{\gamma}}{12 m_{W}^{2}}\right) \\
& +\frac{g^{2} g^{\prime 2}}{\left(m_{W}+\xi^{1 / 2} m_{\gamma}\right)^{4}}\left(\frac{29 \xi m_{W}}{48}+\frac{5 m_{W}^{3}}{48 m_{\gamma}^{2}}+\frac{5 \xi^{1 / 2} m_{W}^{2}}{12 m_{\gamma}}+\frac{\xi^{3 / 2} m_{\gamma}}{3}\right) \\
& -\frac{g^{2} g^{\prime 2}}{\left(\xi^{1 / 2} m_{W}+m_{\gamma}\right)^{3}}\left(\frac{3 \xi}{32}+\frac{9 \xi^{1 / 2} m_{\gamma}}{32 m_{W}}+\frac{m_{\gamma}^{2}}{4 m_{W}^{2}}\right)+\frac{g^{2} g^{\prime 2}}{\left(m_{W}+\xi^{1 / 2} m_{\gamma}\right)^{3}}\left(\frac{7 \xi}{4}+\frac{21 m_{W}^{2}}{32 m_{\gamma}^{2}}+\frac{63 \xi^{1 / 2} m_{W}}{32 m_{\gamma}}\right) \\
& +\frac{g^{2} g^{\prime 2}}{\left(m_{W}+\xi^{1 / 2} m_{\gamma}\right)^{2}}\left(\frac{\xi}{2 m_{W}}+\frac{3 m_{W}}{8 m_{\gamma}^{2}}+\frac{3 \xi^{1 / 2}}{4 m_{\gamma}}+\frac{\xi^{3 / 2} m_{\gamma}}{4 m_{W}^{2}}\right) \\
& \left.-\frac{g^{2} g^{\prime 2} \xi^{1 / 2}}{\left(m_{W}+m_{\gamma}\right)^{2}}\left(\frac{1}{2 m_{W}}+\frac{3 m_{W}}{8 m_{\gamma}^{2}}+\frac{3}{4 m_{\gamma}}+\frac{m_{\gamma}}{4 m_{W}^{2}}\right)\right)-\frac{109(e B)^{2} \zeta(3) g^{2}}{768 T^{2} \pi^{4}} \\
\Pi_{e}^{W}= & \frac{g^{2} T^{2}}{12}+\frac{(e B)^{2} g^{2} \zeta(3)}{256 \pi^{4} T^{2}} \\
\Pi_{f}^{W}= & \frac{g^{2} T^{2}}{4}+\frac{(e B)^{2} g^{2}}{\pi^{4} T^{2}}\left(\frac{133 \zeta(3)}{2304}-\frac{31 \zeta(5)\left(m_{b}^{2}+4 m_{t}^{2}\right)}{1536 \pi^{2} T^{2}}\right) \\
\Pi_{g}^{W}= & -\frac{g^{2} T^{2}}{12}-\frac{g^{2}(e B)^{2} \zeta(3)}{256 \pi^{4} T^{2}}
\end{aligned}
$$

\section{APPENDIX C: GHOSTS SELF-ENERGIES IN ARBITRARY GAUGE}

In this appendix, we list the results for the self-energy diagrams depicted in Fig. 6.

$\gamma$ boson associated ghost $\left(\eta_{\gamma}\right)$ self-energy in arbitrary gauge

$$
\Pi_{a}^{\eta_{\gamma}}=\Pi_{b}^{\eta_{\gamma}}=0
$$

$Z$ boson associated ghost $\left(\eta_{Z}\right)$ self-energy in arbitrary gauge

$$
\begin{aligned}
\Pi_{a}^{\eta_{Z}}= & 0 \\
\Pi_{b}^{\eta_{Z}}= & \frac{g\left(g^{2}-g^{\prime 2}\right)}{\left(g^{2}+g^{\prime 2}\right)^{1 / 2}} \frac{\xi^{2} T m_{Z}}{8 \pi}\left(\frac{m_{W}}{m_{1}+\xi^{1 / 2} m_{W}}-\frac{\left(g^{2}+g^{\prime 2}\right)^{3 / 2}}{g\left(g^{2}-g^{\prime 2}\right)} \frac{m_{Z}}{2\left(m_{4}+\xi^{1 / 2} m_{Z}\right)}\right. \\
& \left.-(e B)^{2} \frac{\xi^{1 / 2}\left(m_{1}^{2}+\xi^{1 / 2} m_{1} m_{W}+\xi m_{W}^{2}\right)}{96 m_{1}^{3} m_{W}^{2}\left(m_{1}+\xi^{1 / 2} m_{W}\right)}\right)
\end{aligned}
$$


$W$ boson associated ghost $\left(\eta_{W}\right)$ self-energy in arbitrary gauge

$$
\begin{aligned}
\Pi_{a}^{\eta_{W}}= & 0 \\
\Pi_{b}^{\eta_{W}}= & \frac{g^{2} \xi^{2} T m_{W}}{16 \pi}\left(\frac{m_{W}}{m_{3}+\xi^{1 / 2} m_{W}}-\frac{m_{W}}{m_{4}+\xi^{1 / 2} m_{W}}+\frac{g^{2}-g^{\prime 2}}{g\left(g^{2}+g^{\prime 2}\right)^{1 / 2}} \frac{m_{Z}}{m_{1}+\xi^{1 / 2} m_{Z}}\right) \\
& +(e B)^{2} \frac{g^{2} \xi^{1 / 2} T m_{W}}{384 \pi}\left(\frac{\left(m_{4}^{2}+4 \xi^{1 / 2} m_{4} m_{W}+7 \xi m_{W}^{2}\right)}{m_{W}^{2}\left(m_{4}+\xi^{1 / 2} m_{W}\right)^{4}}-\frac{\left(g^{2}-g^{\prime 2}\right)}{g\left(g^{2}+g^{\prime 2}\right)^{1 / 2}} \frac{\xi^{3 / 2} m_{Z}\left(7 m_{1}^{2}+4 \xi^{1 / 2} m_{1} m_{Z}+\xi m_{Z}^{2}\right)}{m_{1}^{3}\left(m_{1}+\xi^{1 / 2} m_{Z}\right)^{4}}\right. \\
& \left.+\frac{2\left(m_{3}^{2}+4 \xi^{1 / 2} m_{3} m_{W}+\xi m_{W}^{2}\right)}{m_{W}^{2}\left(m_{3}+\xi^{1 / 2} m_{W}\right)^{4}}-\frac{3\left(m_{3}+3 \xi^{1 / 2} m_{W}\right)}{m_{W}^{2}\left(m_{3}+\xi^{1 / 2} m_{W}\right)^{3}}\right)
\end{aligned}
$$

[1] A.D. Sakharov, Pis'ma Zh. Éksp. Teor. Fiz. 5, 32 (1967); [JETP Lett. 5, 24 (1967)]; Sov. Usp. 34, 32 (1991).

[2] M.B. Gavela, P. Hernández, J. Orloff and O. Pène, Mod. Phys. Lett. A 9, 795 (1994).

[3] K. Kajantie, M. Laine, K. Rummukainen and M. Shaposhnikov, Nucl. Phys. B 466, 189 (1996).

[4] See for example: G. Piccinelli and A. Ayala, Lect. Notes Phys. 646, 293-308 (2004).

[5] A. Ayala, J. Besprosvany, G. Pallares and G. Piccinelli, Phys. Rev. D64, 123529 (2001); A. Ayala, G. Piccinelli and G. Pallares, Phys. Rev. D66, 103503 (2002), A. Ayala and J. Besprosvany, Nucl. Phys. B651, 211 (2004).

[6] B. Cheng, D. N. Schramm, J. W. Truran, Phys. Rev. D49, 5006 (1994); B. Cheng, A. V. Olinto, D. N. Schramm, J. W. Truran, Phys. Rev. D54, 4714 (1996); T. Kahniashvili, A. G. Tevzadze, B. Ratra, "Phase Transition Generated Cosmological Magnetic Field at Large Scales", arXiv:astro-ph/0907.0197.

[7] S. Wang, Phys. Rev. D81, 023002 (2010).

[8] K. Enqvist, Int. J. Mod. Phys. D 7, 331 (1998).

[9] K. Kajantie, M. Laine, J. Peisa, K. Rummukainen and M. Shaposhnikov, Nucl. Phys. B544, 357 (1999).

[10] R. Fiore, A. Tiesi, L. Masperi and A. Megavand, Mod. Phys. Lett, A 14, 407 (1999).

[11] M. Giovannini and M. E. Shaposhnikov, Phys. Rev. D57, 2186 (1998).

[12] P. Elmfors, K. Enqvist and K. Kainulainen, Phys. Lett. B 440, 269 (1998).

[13] D. Grasso and H.R. Rubinstein, Phys. Rep. 348, 163 (2001).

[14] J.D. Barrow, P. Ferreira and J. Silk, Phys. Rev. Lett.78, 3610 (1997).

[15] J.D. Barrow, R. Maartens and C.G. Tsagas, Phys. Rept. 449, 131-171 (2007); for reviews, see A. D. Dolgov, "Generation of magnetic fields in cosmology", arXiv:astro-ph/0306443 M. Giovannini, Int. J. Mod Phys. D 13, 391 (2004); K. Subramanian, Proc. Sci., MRU (2007) 071 arXiv:0802.2804.

[16] M.S. Turner and L.M. Widrow, Phys. Rev. D37, 2743 (1988); see K. Subramanian, Astron. Nachr. 331, 110 (2010), for a review.

[17] G. Baym, D. Bodeker, L. McLerran, Phys. Rev. D53,
662 (1996); J. Ahonen, K. Enqvist, Phys. Rev. D57, 664 (1998); J. M. Quashnock, A. Loeb, D. N. Spergel, Astrophys. J. Lett. 344, L49 (1989); M. M. Forbes and A. R. Zhitnitsky, Phys. Rev. Lett.85, 5268 (2000); T. Stevens, M. B. Johnson, L. S. Kisslinger, E. M. Henley, W.-Y. P. Wang, M. Burkardt. Phys. Rev. D77, 023501 (2008); T. Stevens and M.B. Johnson, arXiv:1001.3694.

[18] For comprehensive reviews see P.P. Kronberg, Rep. Prog. Phys. 57, 325 (1994); R. Beck, A. Brandedenburg, D. Moss, A. Shukurov and D. Sokoloff, Annu. Rev. Astron. Astrophysics, 34, 155 (1996); C.L. Carilli and G.B. Taylor, Annu. Rev. Astron. Astrophys. 40, 319 (2002); F. Govoni and L. Feretti, Int. J. Mod. Phys. D 13, 1549 (2004).

[19] See K. Subramanian, Astron. Nachr., 327, 403 (2006); R. Durrer, New Astron. Rev. 51, 275 (2007), for reviews.

[20] M. Giovannini, Classical. Quantum. Gravity 23, R1 (2006); S.K. Sethi and K. Subramanian, J. Cosmol. Astropart. Phys. 11 (2009) 021; D. G. Yamazaki, K. Ichiki, T. Kajino and G.J. Mathews, Astrophys. J. 646, 719 (2006).

[21] D. G. Yamazaki, K. Ichiki, T. Kajino and G. J. Mathews, Phys. Rev. D81, 023008 (2010).

[22] For comprehensive reviews on EW baryogenesis see for example: M. Trodden, Rev. Mod. Phys. 71, 1463 (1999); A. Megevand, Int. J. Mod. Phys. D 9, 733 (2000).

[23] N. Petropoulos, arXiv:hep-ph/0304275

[24] A. Sánchez, A. Ayala and G. Piccinelli, Phys. Rev. D 75 043004 (2007).

[25] V. Skalozub and V. Demchik, arXiv:hep-th/9912071

[26] V. Skalozub and M. Bordag, Int. J. Mod. Phys. A 15, 349 (2000).

[27] M.E. Carrington, Phys. Rev. D45, 2933 (1992).

[28] P. Arnold and O. Espinosa Phys. Rev. D47, 3546 (1993).

[29] A. Erdas, arXiv:hep-ph/1006.5067.

[30] L. Dolan and R. Jackiw, Phys. Rev. D9, 3320 (1974).

[31] N. K. Nielsen, Nucl. Phys. B 101 , 173 (1975).

[32] J. Schwinger, Phys. Rev. 82, 664 (1951).

[33] A. Ayala, A. Sánchez, G. Piccinelli and S. Sahu, Phys. Rev. D71, 023004 (2005); D. Grasso and H.R. Rubinstein, Phys. Lett. B 379 73, (1996).

[34] T.-K. Chyi, C.-W. Hwang, W.F. Kao, G.L. Lin, K.-W. 
Ng and J.-J. Tseng, Phys. Rev. D 62, 105014, (2000).

[35] M.E. Tejeda-Yeomans, J. Navarro, A. Sanchez, G. Piccinelli and A. Ayala, in Proceedings of the XI Mexican Workshop on Particles and Fields, edited by H. CastillaValdez, O. Miranda, and E. Santos, AIP Conf. Proc. No. 1026 (AIP, New York, 2008).

[36] R. Maartens, Pramana 55, 575 (2000); D. Grasso and H.R Rubinstein, Phys. Lett. B379, 73 (1996).

[37] J. Ambjørn and P. Olesen, Nucl. Phys. B315, 606 (1989).

[38] A. Ayala, J. Navarro, A. Sánchez, and M.E. TejedaYeomans, work in progress.

[39] M. Le Bellac Thermal Field Theory, Cambridge University Press (1996).

[40] D.J. Bedingham, arXiv:hep-ph/0011012.

[41] A. Ayala, G. Piccinelli, A. Sánchez, and M.E. Tejeda-
Yeomans, Phys. Rev. D78, 096001 (2008).

[42] For a clear discussion of the difference between the infrared and static limits, see H.A. Weldon, Phys. Rev. D47, 594 (1993).

[43] J.C. D'Olivo, J.F. Nieves, S. Sahu, Phys. Rev. D 67, 025018 (2003).

[44] N. Sadooghi and K. Sohrabi Anaraki, Phys. Rev. D. 78, 125019 (2008).

[45] J.F. Nieves and P.B. Pal, Phys. Rev. D39, 652 (1989).

[46] A. Ayala, A. Bashir, A. Raya and A. Sánchez, Phys. Rev. D80, 036005 (2009).

[47] J. Navarro work in progress.

[48] J. Navarro, Ph. D. Thesis, Universidad Nacional Autonoma de Mexico (2010). 